\title{
HIGH FREQUUENCY OBSERVATIONS OF IAPETUS ON THE GREEN BANK TELESCOPE AIDED BY IMPROVEMENTS IN UNDERSTANDING THE \\ TELESCOPE RESPONSE TO WIND
}

\author{
Paul A. Ries \\ Rockville, MD
}

B.A., University of Maryland, 2006

M.A., Univiversity of Vìrginia, 2008

A Disertation Presented to the Graduate

Fuculty of the University of Virginia

in Candidacy for the Degree of

Doctor of Philosophy

Department of Astronomy

University of Virginia

May, 2012

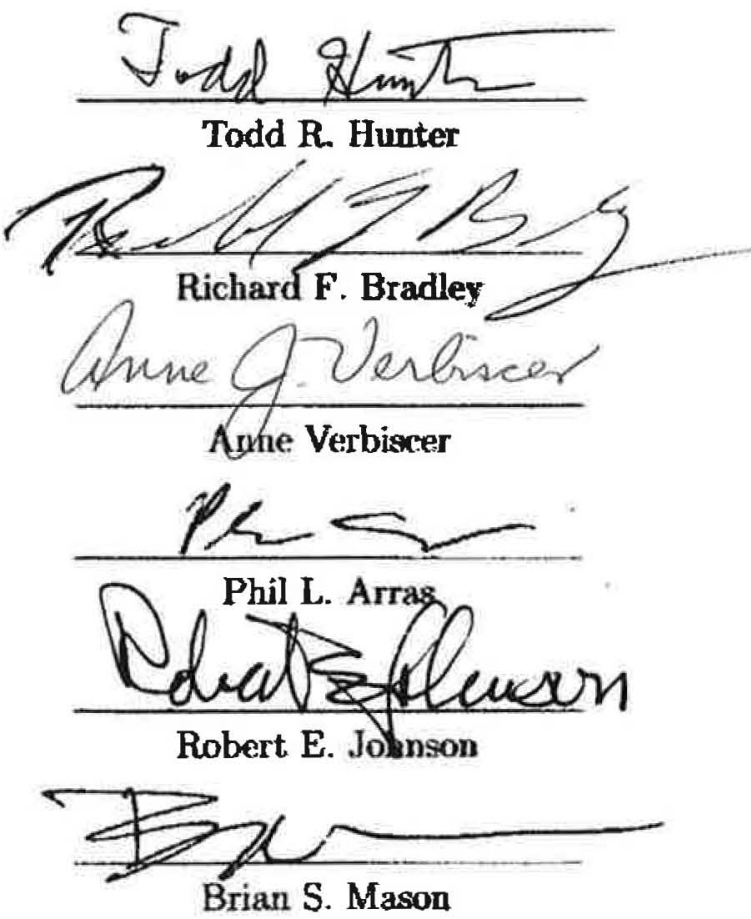


Abstract 
The Green Bank Telescope is a $100 \mathrm{~m}$, fully steerable, single dish radio telescope located in Green Bank, West Virginia and capable of making observations from meter wavelengths to $3 \mathrm{~mm}$. However, observations at wavelengths short of $2 \mathrm{~cm}$ pose significant observational challenges due to pointing and surface errors. The first part of this thesis details efforts to combat wind-induced pointing errors, which reduce by half the amount of time available for high-frequency work on the telescope. The primary tool used for understanding these errors was an optical quadrant detector that monitored the motion of the telescope's feed arm. In this work, a calibration was developed that tied quadrant detector readings directly to telescope pointing error. These readings can be used for single-beam observations in order to determine if the telescope was blown off-source at some point due to wind. With observations with the $3 \mathrm{~mm}$ MUSTANG bolometer array, pointing errors due to wind can mostly be removed $\left(>\frac{2}{3}\right)$ during data reduction.

Iapetus is a moon known for its stark albedo dichotomy, with the leading hemisphere only a tenth as bright as the trailing. In order to investigate this dichotomy, Iapetus was observed repeatedly with the GBT at wavelengths between 3 and $11 \mathrm{~mm}$, with the original intention being to use the data to determine a thermal light-curve. Instead, the data showed incredible wavelength-dependent deviation from a blackbody curve, with an emissivity as low as 0.3 at $9 \mathrm{~mm}$. Numerous techniques were used to demonstrate that this low emissivity is a physical phenomenon rather than an observational one, including some using the quadrant detector to make sure the low emissivities are not due to being blown off source. This emissivity is the among the lowest ever detected in the solar system, but can be achieved using physically realistic ice models that are also used to model microwave emission from snowpacks and glaciers on Earth. These models indicate that the trailing hemisphere contains a 
scattering layer of depth $100 \mathrm{~cm}$ and grain size of 1-2 mm. The leading hemisphere is shown to exhibit a thermal depth effect. 
Acknowledgements 
This thesis is dedicated to everyone who made this possible, as all theses inevitably are. First I'd like to thank those who started me on and guided me down the path of astronomy. I would like to thank my high school friend, Erica M., who may not realize that she inadvertently started me on the path to astronomy. I'd like to thank my very first advisor, Dan Pascu at USNO for employing me for 4 summers and for opening my eyes to how much about our backyard, the solar system is still full of unknowns. Of all the teachers I have had, the one to whom I owe the most thanks is Andy Harris at UMD, from whose radio class I took away a love of all things Fourier, not to mention radio astronomy itself. I'd like to thank my undergraduate thesis advisor at UMD, Doug Hamilton, for allowing me to enjoy a brief stint in the wonderful world of theory, which will only make brief cameos in this work. I would like to thank Rich Bradley for introducing me to radio instrumentation research. I'd like to thank all of my thesis committee members for their valuable input over the past few years. Lastly, thank you to Todd Hunter, who has been a patient advisor for the past 5 years, without whose help I would not be writing this thesis right now.

Of course, there are also those in my personal life to whom I am also grateful. I am beyond grateful for all of my bivalve friends from high school, who taught me it was okay to be a nerd/dork/geek. I owe them more than I can ever repay, so a simple acknowledgement in this work is the least that I can give back. Although I also must state that I hope this work will invalidate the so-called "Morgan-Hill Constant."

I also want to acknowledge my good friend Peter from undergrad. While he may have caused the cheese in our fridge to mysteriously disappear, he also made boring intro engineering physics classes tolerable.

As a collective group, I'd like to thank the $\Psi \Phi \operatorname{club}^{1}$ at UVa and BRMRG for helping keep my sanity throughout grad school.

\footnotetext{
${ }^{1}$ Not to be confused with a fraternity, which StudCo has managed to do on several occasions.
} 
It's been a pleasure to know all of the various grad students in my time here at UVa. Collectively, they introduced me to wine, which has helped me overcome occasional bouts of writer's block while making this thesis. Shout-outs go to Kristen, Gail, Dan, Joleen, Jake 1.0, GV, and Nicole for acting as a useful grad student support group. Any graduate student I've met in my time here, but not explicitly mentioned by name is also awesome.

I also would like to point out that Simon 2010 erred in its dedication: Babylon 5 is way better than ST:Deep Space Nine.

On a more serious note, my work was made vastly easier over the years thanks to sharing an office with my classmate Ryan Lynch, who first turned me on to the amazingness that is Python. Not only that, but in his last year here we had a great, mutually beneficial relationship as we both learned new Python tips and tricks that we shared with each other. This collaboration is no doubt at least partially responsible for the fact that we will have both graduated in a timely fashion.

In a similar vein, Python also deserves some acknowledgement being the most excellent programming language ever. If only it had an "import writethesis" command grad school would be so much shorter.

Lastly, I suppose I should also add the obligatory mundane acknowledgements in here as well. This research was supported by an NRAO traineeship grant, two NRAO Student Observing Support grants, and a Virginia Space Grant Consortium fellowship. The National Radio Astronomy Observatory is a facility of the National Science Foundation operated under cooperative agreement by Associated Universities, Inc. 


\section{Table of contents}

Abstract

Acknowledgements $\quad$ v

List of Acronymns $\quad x$

List of Variables xii

1 Introduction $\quad 1$

1.1 Iapetus, the two-faced moon . . . . . . . . . . . . . . 2

1.2 Iapetus in context . . . . . . . . . . . . . . . . 3

1.3 The formation of Iapetus' albedo dichotomy . . . . . . . . . . . . 6

1.4 Thermal emission in the microwave regime . . . . . . . . . . . . 9

1.5 Thermal Depth Effects and Scattering in Vesta . . . . . . . . . . . . 12

1.6 Microwave Emission Elsewhere in the Solar System . . . . . . . . . . 16

1.7 Microwave emission from Iapetus . . . . . . . . . . . . . . . . 18

2 Enabling High Frequency Observations 21

2.1 Introduction . . . . . . . . . . . . . . . . . . . 22

2.2 Instrument . . . . . . . . . . . . . . . . . . . . . . . . . . . 24

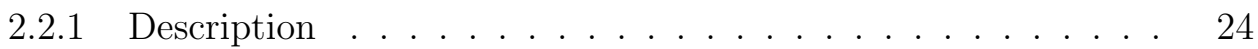

2.2 .2 Data products . . . . . . . . . . . . . . . . . . . . 26

2.2 .3 Calibration . . . . . . . . . . . . . . . . . 26

2.2.4 Median subtraction . . . . . . . . . . . . . . . 31

2.3 Engineering Applcations . . . . . . . . . . . . . . . . . . . . 32

2.4 Astronomical use with MUSTANG . . . . . . . . . . . . . 35

2.5 Other Astronomical Uses . . . . . . . . . . . . . . . . . . . . 37

3 Using the QD with science observations $\quad 48$

3.1 Iapetus Science Data and Wind . . . . . . . . . . . . . . . . . . . 48

3.1.1 Ka-band Observations . . . . . . . . . . . . . . . . . . 49

3.1.2 MUSTANG Observations . . . . . . . . . . . . . . 50

3.2 Additional Science Data . . . . . . . . . . . . . . . . . . 51 
4 Observations $\quad 56$

4.1 Data acquisition . . . . . . . . . . . . . . . . . . . 56

4.1.1 Instrument description . . . . . . . . . . . . . . . . 56

4.1.2 Observing Strategy . . . . . . . . . . . . . . . . . 57

4.2 Data Reduction . . . . . . . . . . . . . . . . . . . . . . 59

4.2.1 Flux Density Calibration . . . . . . . . . . . . . . . . . 59

4.2.2 Saturn as a Sanity Check . . . . . . . . . . . . . . . 67

4.3 Sidelobe Mitigation Technique . . . . . . . . . . . . . . . . . . . . . . 69

4.3.1 Definitions and overview . . . . . . . . . . . . . . . 69

4.3.2 Specific Example: Saturn's moon Iapetus . . . . . . . . . . . . 71

4.3.3 General Sidelobe mitigation technique . . . . . . . . . . . . 77

4.3.4 Application to mapping . . . . . . . . . . . . . . . 77

$4.41 \mathrm{~cm}$ Observational Results . . . . . . . . . . . . . . . . . . . 81

$4.53 \mathrm{~mm}$ Observational Results . . . . . . . . . . . . . . . . . . . 88

5 Modeling $\quad 92$

5.1 Modeling. . . . . . . . . . . . . . . . . . . . . 92

5.1.1 Thermal Modeling . . . . . . . . . . . . . . . . . . 92

5.1.2 Three-tone modeling . . . . . . . . . . . . . . . . . . . 94

5.1.3 SED modeling . . . . . . . . . . . . . . . . . . . 96

5.2 Science Conclusions . . . . . . . . . . . . . . . . . . . . . 103

5.2.1 Variations in Effective Emissivity . . . . . . . . . . . . . 103

5.2 .2 Grain Size . . . . . . . . . . . . . . . . . . . . . . . 105

5.2.3 Informing earlier RADAR results . . . . . . . . . . . 106

5.2.4 Future work .................... 108 
List of Acronymns 


\section{Acronymn Meaning}

\begin{tabular}{|c|c|}
\hline ALMA & Atacama Large Millimeter Array \\
\hline ASTRID & ASTRonomers Integrated Desktop \\
\hline $\mathrm{CCB}$ & Caltech Continuum Backend \\
\hline CIRS & Composite InfraRed Spectrograph \\
\hline DCR & Digital Continuum Receiver \\
\hline FITS & Flexible Image Transport Format \\
\hline GBT & Green Bank Telescope \\
\hline IR & InfraRed \\
\hline ISS & Imaging Science Subsystem \\
\hline JVLA & Jansky Very Large Array \\
\hline $\mathrm{KBO}$ & Kuiper Belt Object (see also TNO) \\
\hline MEMLS & Microwave Emissivity Model for Layered Snowpacks \\
\hline MUSTANG & MUltiplexed SQUID TES Array at Ninety Gigahertz \\
\hline NASA & National Aeronautics and Space Administration \\
\hline NOAA & National Oceanagraphic and Athmosphereic Administration \\
\hline OOF & Out-Of-Focus (Holography) \\
\hline PSD & Position-Sensitive Detector \\
\hline PTCS & Precision Telescope Control System \\
\hline QD & Quadrant Detector \\
\hline SED & Spectral Energy Distribution \\
\hline SKA & Square Kilometer Array \\
\hline STM & Standard Thermal Model \\
\hline TNO & Trans-Neptunian Object \\
\hline VIMS & Visible Infrared Mapping Spectrometer \\
\hline
\end{tabular}


List of Variables 


\begin{tabular}{|c|c|c|}
\hline Variable & Description & Units \\
\hline$A, B, C$ & Arbitrary fitting variables & unitless \\
\hline$A_{\text {bond }}$ & bolometric Bond albedo & dimensionless \\
\hline$a$ & semi-major axis of an orbit & length \\
\hline$B_{\nu}$ & the source function & $\frac{\text { power }}{\text { frequency } \cdot \text { solidangle.area }}$ \\
\hline$c_{P}$ & heat capacity & $\frac{\text { energy }}{\text { mass.temperature }}$ \\
\hline$c$ & the speed of light in a vacuum & $29979245800 \frac{\mathrm{cm}}{\mathrm{s}}$ \\
\hline$D$ & QD calibration matrix & $\operatorname{arcsec}$ \\
\hline$k_{b}$ & the Boltzmann Constant & $1.38066 \times 10^{-16} \frac{\mathrm{ergs}}{\mathrm{K}}$ \\
\hline$K_{T}$ & thermal conductivity & $\frac{\text { power }}{\text { length.temperature }}$ \\
\hline$L_{T}$ & thermal depth & length \\
\hline$L_{\text {sun }}$ & solar luminosity & $3.827 \times 10^{33} \frac{\mathrm{ergs}}{\mathrm{s}}$ \\
\hline$Q D_{\text {corr }}$ & QD with grav. def. removed & unitless \\
\hline$r$ & radius & length \\
\hline$S_{\nu}$ & flux density & $\frac{\text { power }}{\text { frequency } \cdot \text { area }}$ \\
\hline$T$ & temperature & Kelvin (K) \\
\hline$T_{b}$ & brightness temperature & Kelvin (K) \\
\hline$T_{e} q$ & equilibrium temperature & Kelvin $(\mathrm{K})$ \\
\hline$T_{\text {surf }}$ & average surface temperature & Kelvin (K) \\
\hline $\tan \Delta$ & loss tangent & unitless \\
\hline$V$ & Voltage & Volts \\
\hline
\end{tabular}




$\begin{array}{lll}\text { Variable } & \text { Description } & \text { Units } \\ \Delta & \text { distance between two objects } & \text { length } \\ \epsilon & \text { emissivity } & \text { dimensionless } \\ \epsilon_{\mathrm{eff}} & \text { effective emissivity } & \text { dimensionless } \\ \epsilon_{\mathrm{d}} & \text { dielectric constant } & \text { dimensionless } \\ \eta & \text { beaming parameter } & \text { dimensionless } \\ \lambda & \text { wavelength } & \text { length } \\ \nu & \text { frequency } & \frac{\# \text { cycles }}{\text { time }} \\ \Omega & \text { solid angle } & \text { steradians }(\mathrm{sr}) \\ \omega_{\text {rot }} & \text { rotational frequency } & \frac{\text { radians }}{\text { time }} \\ \phi & \text { elevation } & \text { degrees } \\ \rho & \text { density } & \frac{\text { mass }}{\text { volume }} \\ \sigma & \text { standard deviation } & \text { not specified } \\ \sigma_{\mathrm{sb}} & \text { Stefan-Boltzmann constant } & 5.67 \times 10^{-} 5 \frac{\mathrm{ergs}}{\mathrm{cm}^{2} \cdot \mathrm{K}^{4} \cdot \mathrm{sr}} \\ \theta & \text { cross-elevation } & \text { degrees }\end{array}$


Chapter 1

\section{Introduction}




\subsection{Iapetus, the two-faced moon}

Iapetus was discovered by Giovanni Domenico Cassini in October of 1671, but from its discovery, Iapetus was no ordinary moon. Cassini observed the moon at western elongation, but when he looked for it at eastern elongation, he was unable to detect it(Cassini 1677). The reason for the "disappearance" of the moon at eastern elongation was the fact that it changed brightness by two visual magnitudes between those two locations, and thus was too faint to detect at eastern elongation. Cassini (correctly) inferred that this dimming was caused by the fact that Iapetus was tidally locked to Saturn and had one hemisphere which was brighter than the other(van Helden 1984, p. 38) .

Figure 1.1 shows a map of Iapetus' surface. The dark equatorial region region is called the Cassini Regio, after the discoverer of Iapetus, Giovanni Domenico Cassini. The region of the brightest equatorial terrain is divided into two sub-regions, Roncevaux Terra ${ }^{1}$ in the north ${ }^{2}$, and Saragossa Terra in the south. The Roncevaux Terra occupies, but is not quite centered on the trailing side of the moon (Morrison et al. 1986). The polar region is bright throughout all longitudes.

Iapetus' overall density is around $1.1 \mathrm{~g} \mathrm{~cm}^{-3}$ (Thomas 2010), and thus Iapetus is believed to be mostly water ice in bulk composition. Spectral analysis of the Roncevaux Terra shows it to be mostly (> 85\%) water ice on the surface (Clark et al. 2012). However, the Cassini Regio is more diverse in composition, with a mixture of organic materials, hematite $\left(\mathrm{F}_{2} \mathrm{O}_{3}\right)$ and some still-unidentified components (Clark et al. 2012), although it has been well-established that the material contains little to no water ice.

\footnotetext{
${ }^{1}$ Ironically, this icy region is named after a battle in Spain in the August of 778, a geographical and temporal condition antithetical to the formation of ice.

${ }^{2}$ in earlier literature, the Roncevaux Terra described the entire bright trailing hemisphere, not just the northern part.
} 


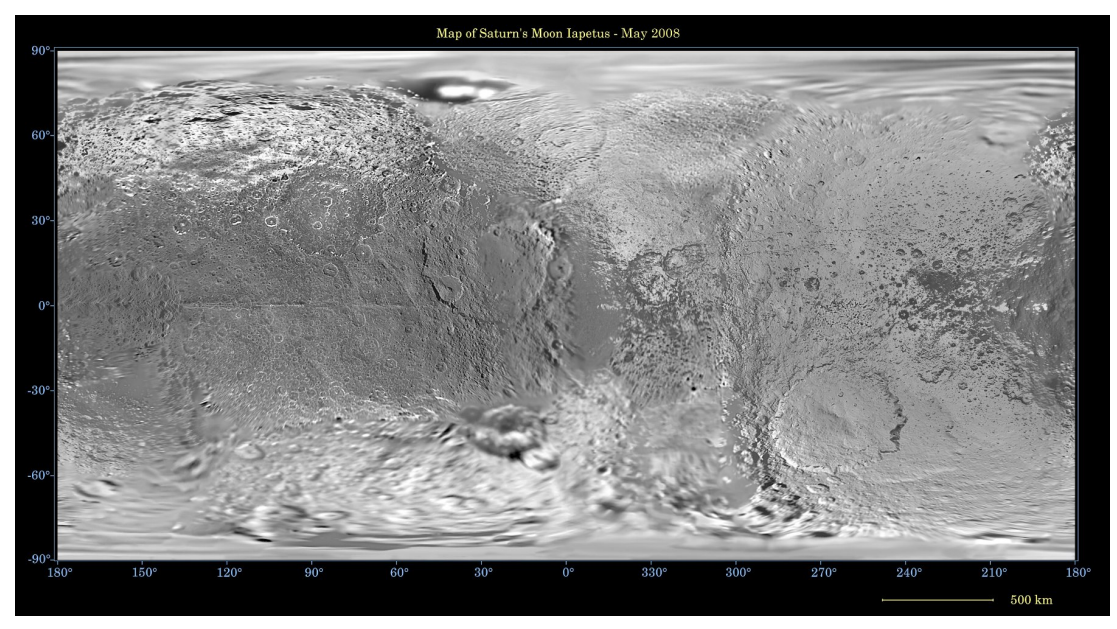

Figure 1.1: Map of Iapetus' surface created with the Cassini's Imaging Science Subsystem (ISS) instrument (Porco et al. 2004, 2005) and with images from the Voyager probe(Smith et al. 1982). The Cassini Regio covers the left 3/4 of the image, and the Roncevaux Terra covers the right 1/4. Image credit:NASA/JPL/Space Science Institute.

How did Iapetus wind up with this two-tone coloration? That is the main science question that this thesis will address, using $3 \mathrm{~mm}$ and $1 \mathrm{~cm}$ radio observations of Iapetus. However, before that question can be addressed, we must place Iapetus in the context of the rest of the solar system and review what information can be gleaned from microwave measurements of solar system surfaces.

\subsection{Iapetus in context}

Table 1.1 compares Iapetus' bulk physical characteristics with several other moons in the solar system. In general, Iapetus has the most in common with the other icy moons of Saturn. Tethys, Rhea, and Dione are of similar size and bulk density as a whole, and albedo in the Roncevaux Terra and Saragossa Terra of Iapetus. However Tethys, Rhea, and Dione are somewhat brighter due to continuously accumulating pristine ice from Saturn's E-ring, which is thought to be debris from Enceladus' 
plumes(Verbiscer et al. 2007). The dark leading albedo (0.05) is radically different from the large icy satellites, however, and more resembles that of Phoebe (0.08). Iapetus is unique among the large icy satellites with the presence of the low-albedo Cassini Regio, and thus considerable effort has been expended in the literature trying to explain the formation of this material.

Table 1.2 gives the orbital properties of several relevant Saturnian satellites. Iapetus' synchronous orbital and rotational periods are quite long (almost 80 days). Iapetus is the most distant of the large Saturnian satellites at nearly three times the orbital distance of Titan. The two most relevant moons to the formation of Iapetus' albedo dichotomy are its nearest significant neighbors of Phoebe and Hyperion. "Nearest" is, however, a relative term, as the ratio of orbital distance of Iapetus to Hyperion is more than a factor of two, and the ratio between Phoebe's orbit and Iapetus' orbit is more than a factor of three. Phoebe's orbit is also retrograde, which has led to suspicion that it may be a captured object (Johnson and Lunine 2005).

The Galilean satellites of Jupiter all have substantially higher bulk densities than Iapetus, which is interpreted as the presence of silicate interiors (and in the case of Europa, perhaps an iron core as well) (Showman and Malhotra 1999). However, Callisto, Ganymede, and Europa all share the same characteristic of surfaces substantially covered in water ice, although with variation in quantity and surface coverage. Callisto's surface contains about 50\% water ice, but it has some ice-free regions (McCord et al. 1998). Europa is completely ice-covered by nearly pure water ice (Showman and Malhotra 1999) at percentages similar to those observed in the Roncevaux Terra of Iapetus. Europa also is thought to have an interior ocean of liquid water; however, this ocean is under an ice crust of at least several km thickness, (Showman and Malhotra 1999) and thus not detectable via surface observations. Ganymede lies somewhere 


\begin{tabular}{|l|l|r|r|r|}
\hline Moon & Host planet & Radius $(\mathrm{km})$ & $\rho\left(\mathrm{g} \mathrm{cm}^{-3}\right)$ & Geom. albedo \\
\hline Enceladus & Saturn & 252.1 & 1.61 & 1.38 \\
Tethys & Saturn & 531.0 & 0.985 & 1.23 \\
Dione & Saturn & 561.4 & 1.48 & 1.00 \\
Rhea & Saturn & 763.5 & 1.24 & 0.95 \\
Titan & Saturn & 2575 & 1.88 & 0.21 \\
Hyperion & Saturn & 135 & 0.54 & $0.2-0.3$ \\
Iapetus & Saturn & $\mathbf{7 3 4 . 3}$ & $\mathbf{1 . 0 8 8}$ & $\mathbf{0 . 0 5 - 0 . 5}$ \\
Phoebe & Saturn & 106.5 & 1.64 & 0.086 \\
Callisto & Jupiter & 2403 & 1.85 & 0.20 \\
Ganymede & Jupiter & 2634 & 1.94 & 0.42 \\
Europa & Jupiter & 1565 & 3.02 & 0.64 \\
Triton & Neptune & 1352 & 2.05 & 0.7 \\
\hline
\end{tabular}

Table 1.1: Physical data for various moons with some similarity to Iapetus. Radii given are mean radii, as some objects are irregular in shape, particularly Hyperion and Phoebe. Radii and densities for Saturnian satellites are taken from Thomas (2010) except for Titan. Albedos for Saturnian satellites are taken from Verbiscer et al. (2007) at $0.5 \mu \mathrm{m}$, except for Titan, Phoebe, Iapetus, and Hyperion. Phoebe albedo from Miller et al. (2011). Remaining data is taken from Table 1.5 of de Pater and Lissauer (2001), which is mostly data from Yoder (1995).

\begin{tabular}{|l|r|r|r|r|}
\hline Moon & $\mathrm{a}\left(\mathrm{R}_{\text {sat }}\right)$ & Period (days) & $\mathrm{e}$ & $\mathrm{i}$ \\
\hline Enceladus & 4.16 & 1.37 & 0.0000 & 0.00 \\
Tethys & 5.15 & 1.89 & 0.0001 & 1.09 \\
Dione & 6.59 & 2.74 & 0.0022 & 0.03 \\
Rhea & 9.21 & 4.52 & 0.0002 & 0.0 .33 \\
Titan & 21.35 & 15.95 & 0.0288 & 0.31 \\
Hyperion & 26.22 & 21.28 & 0.0232 & 0.61 \\
Iapetus & $\mathbf{6 2 . 2 1}$ & $\mathbf{7 9 . 3 3}$ & $\mathbf{0 . 0 2 9 3}$ & $\mathbf{8 . 3 0}$ \\
Phoebe & 226.20 & 548.02 & 0.1634 & 175.24 \\
\hline
\end{tabular}

Table 1.2: Orbital data for several moons of Saturn from http://ssd.jpl.nasa.gov/?sat_elem which uses averages of the values from the SAT 339 JPL ephemerides (Jacobson, R.A. 2010). Orbital distances converted into Saturn radii using a Saturn radius of $57240 \mathrm{~km}$ (see section 4.4 .2 of this work for the origin of this value). For the most part, satellite orbital and rotational periods are synchronous. The only two exceptions on this list are Hyperion (which has a chaotic rotation) and Phoebe, with a rotation period of 0.387 days (de Pater and Lissauer 2001). 
in between Europa and Callisto, completely covered by material composed of at least $50 \% \mathrm{H}_{2} \mathrm{O}$ ice (Showman and Malhotra 1999) which is more than Callisto, but less pure ice than the surface of Europa or the Roncevaux Terra of Iapetus. Despite their differences with Iapetus, Callisto, Ganymede, and Europa are useful objects for comparison, as they are the only icy surfaces in the solar system with substantial study at microwave wavelengths.

\subsection{The formation of Iapetus' albedo dichotomy}

Considerable effort has been spent trying to understand how Iapetus' albedo dichotomy developed, as no comparable feature exists elsewhere in the solar system. Several attempts have been made to "categorize" various explanations, but they are each really rather unique. One of the earliest explanations postulated for the formation of the albedo dichotomy was that Iapetus was a dark surface coated in a thin layer of ice (Cook and Franklin 1970). According to this mechanism, interplanetary meteorites then pulverized the ice layer preferentially on the leading side (Cook and Franklin 1970) and were darker than the icy surface as well. Almost all proposed mechanisms for the formation of Iapetus' albedo dichotomy involve something striking Iapetus, as the combination of orbital motion and tidal lock of Iapetus mean that the minima and maxima of strike probability and minima and maxima of strike velocity will be centered on the trailing and leading hemispheres.

The next theory proposed to explain the albedo dichotomy also involved the impact of interplanetary meteoroids, only icy ones instead of rocky ones (Peterson 1975). The idea behind this theory was that more of the icy meteorites would sublimate on impact with the leading side due to the higher impact velocities, and thus ice would be differentially deposited on the trailing hemisphere on the otherwise dark surface 
of Iapetus (Peterson 1975). Burns et al. (1979) demonstrated that dynamically, dust grains from Phoebe could be a source for the dark material, which was further supported by later modeling (Burns et al. 1996). All of these theories of formation from the albedo dichotomy came before the surface of Iapetus was ever imaged.

In 1980 and 1981, the Voyager probes flew past Saturn and, for the first time, provided resolved images of the surface of Iapetus (Smith et al. 1982). The authors of the observing paper also could not resist putting forth their own mechanism. Smith et al. (1982) postulated an endogenous mechanism which had pushed up dark material through the light surface, based on the fact that craters in the light material were dark at the bottom, thus leaving the alignment of the Cassini Regio with the leading hemisphere a mere coincidence. However, later papers (e.g. Cruikshank et al. (1983) pointed out that tides from Saturn would cause such endogenous activity to be centered on the Saturn-facing face, rather than the leading hemisphere. The mechanism of Cruikshank et al. (1983) was similar to that of Cook and Franklin (1970), namely impacts of rocky objects. However, Cruikshank et al. (1983) proposed that the ice layer on Iapetus was thick, and that the dark material was both a combination of infalling object and non-icy material indigenous to Iapetus left behind after ice sublimated upon impact. Bell et al. (1985) expanded on the model of Cruikshank et al. (1983) by successfully modeling the near-IR spectrum of the dark side of Iapetus with a mixture largely composed of hydrated silicates, and additional evidence for this model was provided by color and spectral comparisons in Buratti and Mosher (1995).

The next model proposed involved an unusual combination of endogenous and exogenous sources. Tabak and Young (1989) proposed a $10^{13}-10^{15} \mathrm{~kg}$, high-velocity, cometary impact with Iapetus, the debris from which preferentially landed on the 
leading hemisphere and were spread out in longitude (but not latitude) by an oscillation in rotation period created by the impact. An even more energetic collision was suggested by Matthews (1992), with a $180 \mathrm{~km}$ comet colliding with a protoHyperion. The direction of the collision would be such that it could place debris into an Iapetus-crossing orbit. In this case, the depth of dark material would be of order $100 \mathrm{~m}$ (Marchi et al. 2002), which is incompatible with 21st century RADAR results (described later).

The last of the models proposed before Cassini arrived at Saturn all focused on direct small-particle impacts on Iapetus. Wilson and Sagan (1996) proposed that Iapetus contained a thin icy surface over a thin dark surface, over an icy core. Additional papers cited alternate origins for the dark material (assuming it to be be mostly an exogenous deposit) in a manner similar to that of the original Cook and Franklin (1970) paper, such as Titan (Owen et al. 2001) or small retrograde satellites other than Phoebe (Buratti et al. 2002).

The most recently proposed (and currently accepted) mechanism is that of Spencer and Denk (2010), which is most similar to the model of Cruikshank et al. (1983). Spencer and Denk (2010) demostrate that a slight albedo reddening and darkening caused by infalling material leads to enhanced water ice sublimation (substantially more than the direct sublimation of impact) in the warm equatorial region on the leading hemisphere and leaves behind a darker remnant material (lag). In addition, the process causes some of the sublimated ice to re-deposit itself on the trailing hemisphere and poles, as the expected velocity of the sublimated material leads to a ballistic travel distance a substantial fraction of Iapetus' radius (Spencer and Denk 2010). One important prediction of this model is that the dark material should be a relatively thin later (of order tens of centimeters) overlying material similar to the 
trailing hemisphere. Denk et al. (2010) find that impact craters on the dark leading side leave higher-albedo ejecta which lend additional support to the explanation of Spencer and Denk (2010). In addition, a possible source for the exogenous material is no longer hypothetical, as a vast debris ring from Phoebe has been detected which crosses Iapetus' orbit(Verbiscer et al. 2009). Phoebe debris could be a source of darkening as Phoebe has similar colors and spectral features in the near-infrared to the Cassini Regio, possibly due polycyclic aromatic hydrocarbons (PAHs) (Cruikshank et al. 2008).

Why is this model favored over all of the many models that have come before? The main reason is the sharp contrast between the light and dark terrain revealed by Cassini cannot be explained solely by impacts (Spencer and Denk 2010). The endogenous origin model of Smith et al. (1982) was never widely accepted for the sheer level of coincidence required and that a Saturn-facing feature would be the most probable. As for the previous models with an exogenous component, they all require a gradual transition between the light and the dark regions. Impact rates and velocities form a continuous distribution, and thus features resulting from impacts (an exogenous component) should show a gradual contrast rather than a sharp one, leaving only Spencer and Denk (2010) to explain the sharp contrast.

\subsection{Thermal emission in the microwave regime}

How do the microwave properties of Iapetus play into theories of the formation of the albedo dichotomy? Before that question can be answered, we must first discuss various mechanisms which can influence microwave emission.

The dominant emission mechanism for most solar system objects is thermal blackbody radiation emitted due to temperatures of tens to a few hundred Kelvin, with 
thermal peaks in the infrared at $10-100 \mu \mathrm{m}$. The microwave regime has various different definitions, but in this work it refers to electromagnetic waves with frequencies between $1 \mathrm{GHz}$ and $200 \mathrm{GHz}$, corresponding to wavelengths of $1.5 \mathrm{~mm}-30 \mathrm{~cm}$. Thus microwave observations fall in the Rayleigh-Jeans limit (the portion of a Planck blackbody curve at wavelengths much longer than or frequencies much lower than those of the peak), where one can make a relatively simple conversion between the measured flux density of the object, the size of the object, and the physical surface temperature of the object:

$$
\begin{aligned}
S_{\nu} & =B_{\nu} \Omega \\
\Omega & =\pi \frac{r^{2}}{\Delta^{2}} \\
B_{\nu} & =T_{b} \frac{2 \nu^{2} k_{b}}{c^{2}} \\
T_{b} & =\epsilon_{\mathrm{eff}}(\nu) T_{\text {surf }}
\end{aligned}
$$

where $S_{\nu}$ is the flux density received at Earth, $\nu$ is frequency, $k_{b}$ is the Boltzmann constant, $c$ is the speed of light in a vacuum, $B_{\nu}$ is the energy emitted per time, unit frequency, unit solid angle, $\Omega$ is the solid angle of the object as viewed from Earth, $r$ is the radius of the object (assuming approximately spherical geometry), $T_{b}$ is the brightness temperature, $T_{\text {surf }}$ is the average physical surface temperature, $\Delta$ is the distance of the object from Earth, and $\epsilon_{\text {eff }}$ is the effective emissivity. The object of this thesis is to better understand $\epsilon_{\mathrm{eff}}$ as it pertains to the surfaces of solar system bodies.

Physical temperature can be obtained any number of ways. For example, it can be determined from fitting the width of a spectral line or from a physical model based 
on the distance from the Sun and expected physical characteristics, both methods of which are independent of the observed thermal emission. It can also be directly determined from thermal emission by measuring the location of the peak wavelength of the blackbody spectrum in the infrared. Everything else is a constant or known quantity (e.g. distance from Earth).

A common thermal model used for asteroids is the Standard Thermal Model (STM) originally written up in Spencer et al. (1989). The key insight of this model is that the reference temperature is affected by the temperature distribution across a surface, with a slowly rotating body having a higher temperature away from the sub-earth point than a rapidly rotating body. This model introduces a correction called the beaming factor, $\eta$, to account for the distribution of temperature across a surface. However, while this affects the total flux density from an object, it does not change the overall shape of a blackbody curve in the Rayleigh-Jeans limit.

However, it is important to specify precisely what is meant by physical temperature, and to explain the use of $\epsilon_{\text {eff }}$, an observed quantity, rather than $\epsilon$, a physical quantity. In a physics context, $T_{b}$ is usually defined as $T_{b}=\epsilon(\nu) T$, where $\epsilon$ can have a value of 0 to $1 . \epsilon$ is an intrinsic property of the observed material and is used in many important laws of physics, in particular Kirchhoff's laws, which state that the absorptivity of a material is equal to the emissivity $(\alpha=\epsilon)$ and that the reflectivity and emissivity are complementary $(1-r=\epsilon=\alpha) . \epsilon$ is also invariably fairly close to unity, and thus frequently approximated as 0.8-1.0 (e.g. Mitchell and de Pater (1994) or Moullet et al. (2011)) at microwave frequencies. For Iapetus at infrared bands, the emissivity is frequently taken to be 0.9 (Rivera-Valentin et al. 2011; Kimura et al. 2011). However, in astronomical observations, the true physical temperature is elusive. Any of the above methods of determining physical temperature will only result 
in a disk-averaged surface temperature (usually for the sun-facing side of the target objects, as that is all that we can observe from Earth in the solar system). What if some radiation is blocked at a particular wavelength, or the physical temperature changes? The effective emissivity and brightness temperature would change relative to the reference (surface) blackbody, and thus complicate any efforts to determine size from the thermal emission even though the emissivity itself is more or less constant. Effective emissivity is an especially useful concept since it can be used to compare physical mechanisms across bodies with different physical and brightness temperatures (i.e. at a wide range of distances from the sun).

In the literature, many authors make no distinction between $\epsilon_{\text {eff }}$ and $\epsilon$. For an example, one can look at studies in the infrared of Iapetus. Carvano et al. (2007) has several plots of "emissivity" of various satellites of Saturn, which are actually $\epsilon_{\text {eff }}$. For this reason, they find a value of 1.0 (the observed $T_{b}$ perfectly matches their reference blackbody) for Iapetus, whereas other papers (quite legitimately) use a value of 0.9 (e.g. Kimura et al. (2011)) for $\epsilon$, because while the emission is more or less a perfect blackbody curve, the curve is offset by $10 \%$ from a perfect radiator. For this work, the observational topic of interest is deviations from a blackbody curve, and thus we will compare $T_{b}$ to a reference temperature throughout.

\subsection{Thermal Depth Effects and Scattering in Vesta}

One well-studied object for variations in brightness temperature with wavelength is asteroid 4 Vesta, in the sense that is the one of the few objects with an actual model

fit to the microwave data. Redman et al. (1992) combined many wavelengths of rotationally averaged brightness temperatures into a spectral energy distribution for Vesta, which is reproduced as Figure 1.2. They proposed two effects that can cause 
the brightness temperature to drop below that of a reference blackbody: thermal depth and scattering.

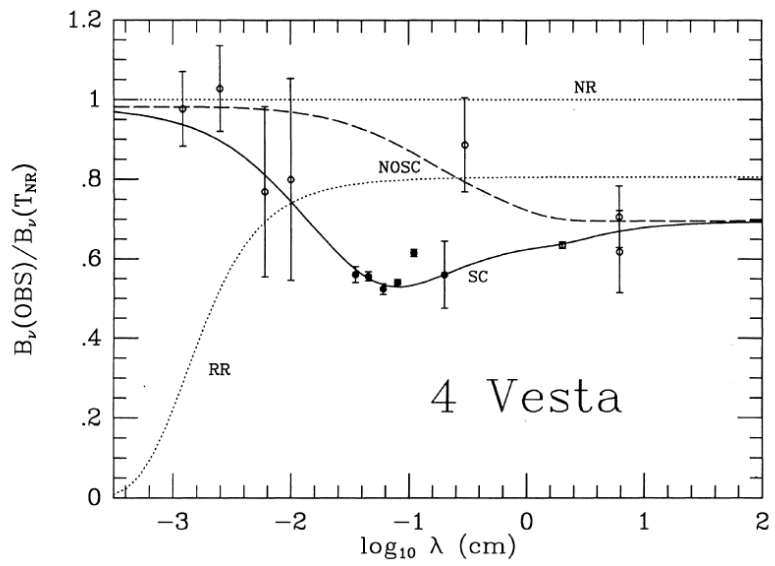

Figure 1.2: Figure 4 from Redman et al. (1992) showing the spectral energy distribution for Vesta. This diagram is essentially a plot of $\epsilon_{\text {eff }}$, where the reference blackbody is that of a non-rotating asteroid (curve labeled "NR"). The dotted "RR" curve is a rapidly rotating asteroid, which has a lower surface temperature and thus deviates from the reference curve. The dashed line (NOSC) is the brightness temperature for a pure surface depth effect and the solid line (SC) is a surface depth effect combined with scattering. Open points are measures published previously to Redman et al. (1992) and the filled points are measurements made by Redman et al. (1992).

The thermal depth effect is fairly simple to understand. Every body in the solar system has an equilibrium temperature which can be calculated from first principles based on assuming thermodynamic equilibrium and setting the incoming and outgoing radiation equal to each other. The result is the following formula for temperature at equilibrium:

$$
T_{\mathrm{eq}}=\frac{1}{2 \sqrt{a}}\left(\frac{L_{\text {sun }}}{\pi \sigma_{\mathrm{sb}}}\left(1-A_{\mathrm{bond}}\right)\right)^{\frac{1}{4}}
$$

where $a$ is the distance between the body and the sun, $L_{\mathrm{sun}}$ is the solar luminosity, $\sigma_{\mathrm{sb}}$ is the Stefan-Boltzmann constant, and $A_{\text {bond }}$ is the bolometric Bond albedo, the total 
reflection of incoming sunlight averaged over all wavelengths. However, this simple formula has a drawback in that while the average surface temperature should be equal to the equilibrium temperature, some parts of the surface will be warmer and some will be cooler. The main driver of the surface temperature distribution on an airless body is solar heating. During their daytimes, the surfaces of solar system bodies are heated by the sun. As a result, the surface becomes hotter than the immediate subsurface. This heat gradually propagates down deeper over the course of the day, forming what is known as the diurnal wave (see e.g. (Muhleman 1972)). The depth can be calculated using the following formula from Muhleman (1972):

$$
L_{T}=\sqrt{\frac{2 K_{T}}{\omega_{\text {rot }} \rho c_{P}}}
$$

where $L_{T}$ is the thermal skin depth, $K_{T}$ is the thermal conductivity of the surface material, $\omega_{\text {rot }}$ is the solar rotation frequency of the object being studied, $\rho$ is the density of the surface material, and $c_{P}$ is the heat capacity of the surface material. For example, one could calculate the thermal depth of Earth's moon, since the moon's thermal, physical, and electromagnetic properties are well-known. Apollo 15 actually conducted an experiment to measure the thermal conductivity of the lunar surface (Keihm et al. 1973) at Hadley Rille. From their model, the expected values at a depth of $5 \mathrm{~cm}$ is $1.5 \mathrm{~g} \mathrm{~cm}^{-3}$ for density (Heiken et al. 1991), $0.7 \mathrm{~J} \mathrm{~g}^{-1} \mathrm{~K}^{-1}$ for heat capacity at $300 \mathrm{~K}$, thermal conductivity of $5 \times 10^{-5} \mathrm{~W} \mathrm{~cm}{ }^{-1} \mathrm{~K}^{-1}$, and $\omega_{\text {rot }}$ of $2.5 \times 10^{-6}$ radians $\mathrm{s}^{-1}$ (Keihm et al. 1973) ${ }^{3}$. Thus the expected thermal depth for the moon is about $6 \mathrm{~cm}$. The thermal depth gives an $e$-folding depth such that

\footnotetext{
${ }^{3}$ The values of $\rho$ and $K_{T}$ on the moon change substantially with depth(Keihm et al. 1973). The answer here will thus be a bit of an overestimate since thermal conductivity is substantially lower on the surface than at $5 \mathrm{~cm}$ depth.
} 


$$
T(x)=\left(T_{\text {surf }}-T_{\text {eq }}\right) e^{-\frac{x}{L_{T}}}+T_{\text {eq }}
$$

where $x$ is the depth. This phenomenon leads to a drop in effective emissivity as observations go to longer wavelengths because longer wavelengths observe at greater depth (penetration depth $\approx 10-30 \lambda$ ). A drop in brightness temperature occurs as the the observed depth passes through the diurnal wave. Eventually, the wavelength is long enough that the observation is measuring deeper than the diurnal wave, resulting in a brightness temperature equal to that of the equilibrium temperature of the object (i.e. the brightness temperature declines from the surface temperature, then levels off at the equilibrium temperature as wavelength increases). On the night side, the thermal wave dissipates or may invert (i.e. brightness temperature is flat or increases gradually to level off at the equilibrium temperature going to longer wavelengths). Examples of such models include those of Muhleman (1972) or Mitchell and de Pater (1994).

Scattering results in a characteristic drop in brightness temperature due to the interplay between wavelength, particle size, and particle optical properties. Vesta is the prototypical case for this phenomenon in the astronomical literature (Redman et al. 1992), as scattering can be differentiated from a thermal depth effect by the fact that it forms a u-shape in effective emissivity (i.e. it declines, levels, then increases with increasing wavelength). A combination of scattering and a thermal depth effect are required to explain the spectral energy distribution seen in Fig. 1.2. Unfortunately, Redman et al. (1992) states that the model they used to produce the graphs in that figure will be the subject of an upcoming companion paper. Twenty years later, that paper has yet to appear. ${ }^{4}$

\footnotetext{
${ }^{4}$ Some slightly more detailed description of of scattering was provided in (Redman et al. 1998), but not at a level where one could actually generate a model of brightness temperature vs. wavelength.
} 


\subsection{Microwave Emission Elsewhere in the Solar System}

Vesta is the only asteroid which requires substantial scattering to explain its brightness temperature. Mueller and Lagerros (1998) produced a thermophysical model for asteroids which is still commonly used and noted that the drop in brightness temperature of the magnitude observed on Vesta was not observed in the other large asteroids they modeled. However, the scattering model for Vesta ran into an unexpected complication when a $33 \%$ variation in its rotational light-curve was detected at $3 \mathrm{~mm}$ wavelength (Müller and Barnes 2007). This change in the brightness temperature with rotational phase was suggested to be due to a suspected nearby large crater. Unfortunately this hypothesis has not survived the arrival of the Dawn spacecraft at Vesta, where no such large crater was found.

Do other asteroids show a change in brightness temperature with rotational phase? This question cannot be answered at this point because only a handful of asteroids have rotational light-curves in the thermal $\mathrm{mm}$ or submm regime (or searches for them) published in the literature and all of them are disputed to some extent. The only other claimed detection is for a $50 \%$ light curve for Ceres and a $20 \%$ one for Pallas at $870 \mu \mathrm{m}$ (Chamberlain et al. 2009), but the result for Ceres is strongly contested by Moullet et al. (2010) which found variation only at the few\% level at $1.3 \mathrm{~mm}$.

As for the other large population of small solar system bodies, the Kuiper Belt has had few $\mathrm{cm}, \mathrm{mm}$, or submm wave detections thus far. However, the prototypical TNO, Pluto, was the subject of substantial discussion due again to differing brightness temperatures in the infrared and the millimeter. The Pluto-Charon system was first observed at millimeter wavelengths in 1988 (Altenhoff et al. 1988) at $1.2 \mathrm{~mm}$ 
with a flux density of $15.0 \pm 1.4 \mathrm{mJy}$. This detection was highly significant, because the inferred temperature from these measurements $(\approx 40 \mathrm{~K})$ was significantly different from that inferred from measurements at 60 and $100 \mu \mathrm{m}(\approx 60 \mathrm{~K})$ obtained by Aumann and Walker (1987) with the IRAS satellite. Jewitt (1994) tried to explain this discrepancy with longitudinal temperature variations. Tryka et al. (1994) explains this variation with a model of $\mathrm{N}_{2}$ polar caps with low temperature and a low albedo, $\mathrm{N}_{2}$-free equatorial region. These models somewhat overestimate the flux in the millimeter region; however, since $\mathrm{N}_{2}$ ice is believed to have non-uniform emissivity in the millimeter bands, this discrepancy can be corrected to match observations (Stansberry et al. 1996). This lower emissivity for ices is in essence indistinguishable from the scattering effect detected by Redman et al. (1992).

What about the major planets? Mercury shows a brightness temperature drop at long wavelengths attributed to a thermal depth effect(Mitchell and de Pater 1994). The case of Earth is highly terrain-dependent, and will be discussed in great detail in a later chapter. Mars is almost a perfect blackbody in the microwave (Cuzzi and Muhleman 1972). As for the gas giant planets, they are dominated by pressurebroadened absorption lines. Saturn's rings deviate substantially from a blackbody due to scattering(Muhleman and Berge 1991), but their relative contribution compared to the planet itself is small.

The Earth's moon is certainly worth mentioning again. It was where the first drop in brightness temperature at long wavelengths attributed to a thermal depth effect was detected in the solar system(Tikhonova and Troitski 1969; Linsky 1966). Furthermore, the microwave light-curve of the Moon is known to lag the infrared light-curve, due to the fact that it takes longer for heat to penetrate deeper in the lunar surface (Linsky 1966; Muhleman 1972; Keihm 1984). Furthermore, this lag 
increases at longer wavelengths, with the lag at $1.2 \mathrm{~mm}$ equal to $16^{\circ}$ increasing to $44^{\circ}$ at $3.2 \mathrm{~cm}$ as the depth probed by microwave emission increases (Linsky 1966).

Most moons have not been observed extensively in the microwave regime, but the four Galilean satellites of Jupiter have extensive data (Muhleman and Berge 1991) of particular interest because they are among the few icy, airless bodies ever observed in the microwave. Io and Callisto's brightness temperatures are reasonably explained with a surface depth effect. However Ganymede and especially Europa displayed unusually low brightness temperatures, with Europa having a brightness temperature of only $50 \mathrm{~K}$, a surface temperature of $120 \mathrm{~K}$, and an equilibrium temperature of $90 \mathrm{~K}$. Muhleman and Berge (1991) invoked scattering in order to explain the decline in brightness temperature across a wide swath of the microwave spectrum which was far too low to possibly correspond to a physical temperature, although no quantitative modeling was performed. One important trend across the Galilean satellites is that the observed microwave brightness temperature is anti-correlated with the amount of ice presence (i.e. Io has the least ice and highest $\mathrm{T}_{b}$, and Europa has the most ice and lowest $\mathrm{T}_{b}$ ).

\subsection{Microwave emission from Iapetus}

So why observe Iapetus' microwave emission? First, one would expect there to be substantial variation in temperature due to the dark material being hotter than the light material. Variation in thermal emission from Iapetus has been detected previously as early as 1972(Murphy et al. 1972), however, no thermal light-curve for Iapetus has ever been directly observed. While the Cassini spacecraft produces excellent thermal

data, it cannot produce a thermal light curve for Iapetus due to the combination of Iapetus' long rotation period (80 days)and the short duration of a flyby (1 day), at 
least the type of light-curve typically measured from Earth (the variation of sub-solar temperature with longitude). Additionally, Iapetus is tidally locked to Saturn, which prevents Cassini from making observations of the anti-Saturn hemisphere while orbiting close to Saturn, as it will for the remainder of the mission. Snapshots of Iapetus' surface with the Composite Infrared Spectrograph (CIRS) instrument during Iapetus flybys allow for a complete thermal map of the surface (Howett et al. 2010), but not the thermal lightcurve.

However, as noted in the sections above, many bodies in the solar system show non-thermal characteristics in the microwave regime. One important constraint on the models for the albedo dichotomy of Iapetus is the fact that the leading and trailing hemispheres should be similar at a certain depth. Spencer and Denk (2010) and Tamayo et al. (2011) require a depth of similarity of order tens of centimeters. The theory of Cook and Franklin (1970) required of $1.6 \mathrm{~m}$. The results of Marchi et al. (2002) require one of $100 \mathrm{~m}$. Wilson and Sagan (1996) requires subsurface structure at a few $\mathrm{km}$ below the surface in addition to a thin layer near the surface. As longer wavelengths probe deeper depths, observing over a range of wavelengths can determine at what depth the two hemispheres diverge or converge in composition. This measurement is already constrained by RADAR reflection observations from Cassini (Ostro et al. 2006) at $2.2 \mathrm{~cm}$ and Arecibo at $13 \mathrm{~cm}$ (Black et al. 2004). The leading and trailing albedos diverge at $2.2 \mathrm{~cm}$ but are similar at $13 \mathrm{~cm}$. This result implies that the surfaces are similar at a depth on order of a meter. Measurements of microwave emission (brightness temperature and effective emissivity) are complementary to RADAR reflectance measurements and should provide a similar constraint on depth of similarity and subsurface structure. In addition, microwave emission can be measured at shorter and more numerous wavelengths than RADAR reflectance, thus 
opening a wavelength regime (and subsurface depths) that has never been studied for Iapetus.

Therefore, we set out to obtain a microwave light-curve of Iapetus to constrain the thermal and/or scattering properties of Iapetus' surface. 
Chapter 2

Enabling High Frequency

Observations 


\subsection{Introduction}

Before work began on the science part of this thesis, substantial effort was spent on characterizing and improving the high-frequency performance of the telescope that was used to obtain science data. Located at an elevation of $822 \mathrm{~m}$ in Green Bank, West Virginia, the Green Bank Telescope (GBT) (Prestage et al. 2009) is a 100m fully steerable single-dish radio telescope. The optical design is a dual offset Gregorian on an alt-az mount providing an unblocked aperture of $100 \mathrm{~m}$ diameter. The primary paraboloid is formed by a segmented active surface of 2004 panels (Lacasse 1998) which enables observations from frequencies of $300 \mathrm{MHz}$ to $115 \mathrm{GHz}$. The highfrequency receivers $(\nu>1.15 \mathrm{GHz})$ illuminate the concave ellipsoidal subreflector from a receiver cabin at the Gregorian focus (Jewell and Prestage 2004). Figure 2.1 shows a profile diagram of the telescope. At high frequencies (above $50 \mathrm{GHz}$ ), the diffraction beam size of the GBT $\left(\approx \frac{740^{\prime \prime}}{\nu(\mathrm{GHz})}\right)$ is less than $15^{\prime \prime}$, so observations become particularly sensitive to uncorrected pointing and tracking errors. Indeed, the two primary telescope-related performance factors that limit high-frequency observing are pointing accuracy and surface accuracy. ${ }^{1}$

In order to improve the GBT pointing and surface performance, the Precision Telescope Control System (PTCS) project was initiated in early 2003. It is wellknown that changes in the thermal environment of large radio telescopes have a considerable effect on the pointing (Bayley et al. 1994). Therefore, the first major PTCS deliverable was the implementation of a structural temperature network and inclinometer system to allow dynamic corrections to augment the static pointing model based on real-time measurements (Constantikes 2004; Prestage et al. 2004). Following the recent upgrade of the telescope azimuth track (Symmes et al. 2008), this

\footnotetext{
${ }^{1}$ Much of the text and figures in this chapter are taken from (Ries et al. 2011).
} 
system has produced an all-sky blind pointing performance in low winds $\left(<3 \mathrm{~m} \mathrm{~s}^{-1}\right)$ of 5" rms, or about half the diffraction beamsize at $90 \mathrm{GHz}$ (Constantikes 2007; Prestage et al. 2009). The second major PTCS effort was the application of the technique of out-of-focus (OOF) holography (Nikolic et al. 2007a) which succeeded in measuring the large-scale gravitational deformation of the surface that remains after application of the finite-element model. This technique yielded a set of elevation-dependent Zernike coefficients (through fifth order) which are now automatically applied and produce a nearly constant telescope gain vs. elevation during nighttime observations (Nikolic et al. 2007b). In 2008, the OOF measurement and active surface correction sequence was automated to allow fine tuning of the surface collimation with minimal overhead, thereby expanding the possibility of high-frequency daytime observations during which the telescope thermal environment can change quickly (Hunter et al. 2009). Finally, in 2009 the small-scale rms surface error of the GBT was reduced significantly (from 390 to 240 microns rms) by refining the 2,209 actuator home positions during a campaign of traditional radio holographic measurements using a 12 GHz satellite beacon (Hunter et al. 2010). This improvement in surface accuracy resulted in a 240\% increase in the $90 \mathrm{GHz}$ efficiency (Hunter et al. 2011).

Following all of the recent surface improvements, the focus of the PTCS project activity has shifted back to pointing performance. The efforts described in this paper center on measuring and reducing the dynamic pointing error. On the GBT, it is motion of the vertical feedarm that is the largest source of dynamic pointing errors, which we define as those that can occur over the course of observing a single source, i.e. on timescales of seconds to minutes (Smith et al. 2000). Although the GBT feedarm design provides an unblocked aperture, it is less stiff than a traditional onaxis quadrupod design. Motion of the feedarm tip results in a relative movement of 
the subreflector with respect to the primary mirror which appears (to first order) as a pointing error in the focal plane. These deflections can become significant when the feedarm undergoes structural resonance or when the feedarm is buffeted by winds. The wind problem is especially acute for high-frequency observing. High frequency performance depends on good atmospheric transparency and good pointing. At wind speeds above $\sim 4 \mathrm{~m} \mathrm{~s}^{-1}$, the feedarm deflections become the dominant constraint on the high-frequency performance of the telescope. Reducing the wind-induced pointing errors could nearly double the amount of available high-frequency observing time because periods of good transparency can sometimes occur accompanied by significant winds, particularly in winter.

\subsection{Instrument}

\subsubsection{Description}

The approach we have taken to investigate dynamic pointing errors on the GBT is to add a simple feedarm metrology system to the telescope. Although the GBT pointing performance was originally designed to utilize corrections from a complex laser metrology network (see e.g. Parker and Payne 1999), development work on this network was postponed in late 2003 in order to concentrate resources on improving the thermal pointing model and pursuing the more conventional techniques of surface holography. However, by this time, one component of the optical metrology scheme had already reached an advanced stage. This device consists of an optical illuminator aimed at a position sensitive detector (PSD). For historical reasons, we traditionally refer to the aggregate system as a "quadrant detector" (QD) as it was originally conceived by John M. Payne to operate in this manner in the early 1990's (Hall 
et al. 1998). QDs (and more generally PSDs) have had many useful applications in astronomy, such as for optical image stabilization (Stilburn et al. 1997; Stilburn 1992) and X-ray astronomy (Culhane 1991). In our case, the purpose of the QD is to provide two-dimensional positioning information of the feedarm relative to the telescope elevation axle in approximately the same plane as the focal plane of the telescope. Thus, if properly calibrated, its datastream can be used to detect and correct for wind- or resonance-induced pointing errors from the feedarm incurred during astronomical observations.

After testing several generations of prototypes, the current version of the QD was deployed in late 2004. Figure 2.1 shows the location of the illuminator and detector with respect to the general telescope layout. The illuminator assembly is located near the tip of the vertical feedarm, slightly below the subreflector (Figure 2.2). The illuminator source is a Luxeon III Star $1400 \mathrm{~mA}$ green LED. A $150 \mathrm{~mm}$ focal length lens produces a slightly divergent beam in order to maintain illumination of the detector at all possible telescope orientations. A 12-inch hole in one panel of the dish allows light from the illuminator to reach the detector assembly underneath. The hole lies about $10 \mathrm{~m}$ to the right of the symmetry axis of the telescope, which allows the detector assembly to be placed on an accessible platform near the right elevation bearing. Inside the weather-tight detector box is a flat turning mirror followed by a telescope with an $800 \mathrm{~mm}$ focal length lens that focuses the beam onto a $4 \mathrm{~mm}$ square duolateral PSD (Figure 2.2). The total path length of the beam is $\approx 87 \mathrm{~m}$. The PSD produces four voltages, each of which is amplified, filtered by an active filter centered at the modulation frequency, and then detected by an RMS-to-DC converter. The converter output voltages are digitized at $\approx 8.8 \mathrm{~Hz}$ and timestamps are attached by a microcontroller. The digitized signal is then transmitted via an RS-232 serial link to 
a concentrator, which then sends the signal over ethernet back to the control room.

\subsubsection{Data products}

The centroid of the illuminator spot on the surface of the detector is given by the unitless ratio of the differences between the two pairs of PSD output signals to their

sum (i.e. $\left.\mathrm{QD}_{X}=\frac{V_{1}-V_{2}}{V_{1}+V_{2}}, \mathrm{QD}_{Y}=\frac{V_{3}-V_{4}}{V_{3}+V_{4}}\right)$. The PSD is large enough that the illuminator spot is fully captured for all elevations above $15^{\circ}$, but the spot begins to walk off the PSD below that point. This limitation is acceptable because high-frequency observations are not generally attempted at such low elevations due to atmospheric opacity. The GBT monitor and control (M\&C) system collects and stores the QD data into binary FITS tables (Cotton et al. 1995) along with several calibrated data products derived from these voltages (described later). A plug-in tool is available in ASTRID, the GBT user interface software (O'Neil et al. 2006), to graphically display the QD-inferred pointing error in real-time. The running rms of the data is also displayed in text format on the GBT status window which is available to GBT observers and operators in ASTRID. The illuminator and detector assemblies each contain a number of sensors that measure the temperature of the internal air, the electronics, and the optics, which are also timestamped and stored in a separate binary FITS file.

\subsubsection{Calibration}

\section{Overview}

The first step in the calibration process was to remove the signature of gravitational deformation of the feedarm from the QD signal and did so by fitting the raw QD signal at many elevations with a model (described in the next section). The next step 
was to calibrate from raw QD coordinates to a pointing error. We assumed that the physics were linear and described it in the form of a matrix equation:

$$
\underbrace{\left|\begin{array}{cc}
D_{11} & D_{12} \\
D_{21} & D_{22}
\end{array}\right|}_{D} \underbrace{\left|\begin{array}{ll}
Q D_{X, c o r} & \cdots \\
Q D_{Y, \text { cor }} & \cdots
\end{array}\right|}_{p_{\text {corr }}}=\underbrace{\left|\begin{array}{cc}
\delta \phi & \cdots \\
\delta \theta & \cdots
\end{array}\right|}_{\text {err }}
$$

where $\delta \phi$ is the offset in elevation and $\delta \theta$ is the offset in cross-elevation and the QD variables are the QD X and Y after the removal of the signal of gravitational deformation. Mathematically, the $2 \times 2$ matrix represents a combination of rotations and scalings required to convert the raw QD signal with the gravitational deflection removed to pointing errors in elevation and cross-elevation. However, the elevation and cross-elevation calibrations in this formulation are independent of each other. Therefore, we calibrated them using different methods: elevation using a conversion between the surveyed deflection of the feedarm with elevation, and the QD measured motion of the feedarm with respect to elevation and cross-elevation using a direct conversion between astronomical pointing measurements and QD measured pointing errors. The final result of our calibration is given below in units of arcsec:

$$
\left|\begin{array}{ll}
D_{11} & D_{12} \\
D_{21} & D_{22}
\end{array}\right|=\left|\begin{array}{cc}
-550.9 & -156.4 \\
+570.0 & -468
\end{array}\right|
$$

\section{Removal of Gravitational Deflection}

The existing static pointing model for the telescope already accounts for the gravitational deflection of the feedarm, so the deflection must be removed from the QD data in order to isolate the residual dynamic pointing errors. This problem was fairly trivial to resolve. We simply tipped the telescope from approximately 70 to $30^{\circ}$ elevation 
and measured the resulting spot position on the $\mathrm{QD}$ (referred to as $\mathrm{QD}_{X}$ and $\mathrm{QD}_{Y}$, which can range from a unitless -1 to +1 ). We assumed that the deflection was linear in trigonometric functions of elevation $(\phi)$, i.e. of the form:

$$
Q D_{\text {gravdef }}=A \cos (\phi)+B \sin (\phi)+C
$$

and then simply performed a linear least squares fit for the coefficients based on the thousands of data points acquired during a single tip of the telescope. The gravitational deformation is then removed using the equation:

$$
\left|Q D_{\text {corr }}\right|=Q D_{\text {obs }}-(A \cos (\phi)+B \sin (\phi)+C)
$$

for each axis of the telescope, with different coefficients for each axis. Figure 2.3 shows

the results from the tip and the least squares fit. What remains after this subtraction is the relative position of the spot on the QD with respect to the standard "no error" position.

\section{Elevation Calibration: Tipping Survey}

As has been stated previously, the feedarm deflects due to the changing gravity vector. In order to calibrate the QD in situ, we consulted archival data from an elevation tipping survey of the GBT performed on September 7, 2000. This survey used traditional techniques employing a Leica total station instrument to determine the change in position of the feedarm vs. elevation with respect to a fixed point. Measurements were recorded at 6 different elevations from $5^{\circ}$ to $95^{\circ}$ and resulting deflections were

calculated. A fit to the measured deflection of the feedarm location of the illuminator with respect to the detector can be seen in Figure 2.4. This fit gave us a conversion between raw QD signal and the deflection in millimeters of the feedarm. We then cal- 
culated the conversion from millimeters of motion of the feedarm to resulting pointing error on the sky simply based on the geometry of the telescope optics. Combining these two steps gave us the conversion between raw QD signal and pointing error.

Unfortunately, this method has a major drawback: the displacement of the feedarm due to an elevation change is primarily in the elevation direction. Since the crosselevation direction is, by definition, perpendicular to the gravity vector at all elevations, the feedarm does not change in position substantially in cross-elevation as the telescope moves in elevation. As a result, the tipping survey leads to a poor fit in cross-elevation since there is almost no change in cross-elevation to fit.

\section{Cross-Elevation Calibration: Astronomical Observations}

An alternate method of calibrating the QD in situ is to compare it directly to the result of "half-power tracks", which measure the dynamic pointing of the telescope. In a half-power track, one observes a bright point source at the half-power point of the telescope beam, offset in either the elevation or cross-elevation directions. The slope of the receiver's beamshape (which is effectively Gaussian due to the typical $14 \mathrm{~dB}$ edge tapered illumination pattern of the feedhorn) at the half-power point gives the derivative of the antenna temperature (i.e. received power) with respect to the change in pointing offset. This slope can then be used to convert the changes in antenna temperature during a track at the half-power point of the beam into pointing error in arcseconds vs. time. By using the slope at the half-power point as the conversion factor, we get a pointing error accurate to within $2 \%$ over a range of $\pm 10^{\prime \prime}$ from the half-power point at $\mathrm{Ku}$-band $(14 \mathrm{GHz})$ where the beamsize is $53^{\prime \prime}$. Atmospheric variations can also cause fluctuations in antenna temperature, however

we can remove most of these fluctuations by differencing the source signal with the 
signal from the second receiver beam which is offset by $330^{\prime \prime}$ in cross-elevation and observing blank sky. As a result, we were able to accurately measure true pointing errors of up to $10^{\prime \prime}$ during the half-power tracks. To extract larger pointing errors, a more rigorous analysis should use the inverse of the error function (Smith et al. 2000); however, our simpler approach was sufficient for our needs.

By analyzing the results of dozens of half-power tracks under different observing conditions, we gathered some general conclusions. Under benign (low-wind, long sidereal tracks on a source or its half-power point) conditions, feedarm motion is not the dominant source of pointing error. We found two conditions under which feedarm motion can dominate: when winds are $>\sim 4-5 \mathrm{~m} \mathrm{~s}^{-1}$ or when structural oscillations are induced in the feedarm by the telescope slews. Because these oscillations decay quickly ( $\approx 60 \mathrm{~s}$ in duration), we modified the half-power track technique to include a periodic series of "kicks" using a custom trajectory. Kicks consist simply of moving the telescope off-source by a substantial fraction of a degree, then back to the halfpower point as rapidly as possible. By spacing the kicks at one minute intervals, the feedarm can be kept nearly constantly oscillating during a half power track. From the result of these tests, it is clear that the feedarm oscillates primarily in the cross elevation direction with very little effect seen in the elevation direction, such as can be seen in Figure 2.5.

A calibration was obtained by doing a direct least squares fit of the QD data to the pointing on the sky during these half-power tracks with kicks. Since the excitation of the feedarm resonance is almost entirely in cross elevation, this method yielded a good and consistent fit in cross-elevation, but poor and inconsistent fits in elevation. We therefore took the cross-elevation calibration from this method and combined it with the earlier elevation calibration from the tipping calibration in order to obtain 
our final overall calibration in both axes.

\subsubsection{Median subtraction}

Over the course of our experiments, we have discovered that, even after calibration, some residual errors exist in the QD signal due to drift in the zero point of the QD signal. The most frequent cause of QD drift is thermal deformation in the QD assembly. A clear diurnal effect is frequently seen in the signal, with the steepest slope of order 4-10 arcsec per hour during the heating and cooling periods. The signal is generally stable after midnight. Figure 2.6 shows QD readings over several days with diurnal variation observed. We solve this problem by taking a median of the QD

data, either before or during the period of interest and subtract the median from the data. Following this correction, the QD data appear to be stable on at least 1 hour timescales, especially at night.

We also occasionally get some hysteresis events in the QD which change the zero point by up to 150 arcseconds and are likely caused by oilcanning in the mount for the detector assembly. An example of such an event can be found in Figure 2.7. However, these events are rare (one every few days) so median subtraction is adequate for removing them from the data. Median subtraction also eliminates any problems of temporal variation in the total refraction along the optical path from the illuminator to the detector.

Median subtraction cannot, however, solve the problem of a loss of QD signal due to a fog, rain, or snow event interrupting the beam. This phenomenon manifests itself in the form of a drop in QD voltage to $60 \%$ or less of its normal value and in the form of enormous errors in the QD reading. Fig. 2.8 shows an example of the QD being interrupted by fog and the resulting spurious readings. Our solution is to simply not 
use the QD when the weather is interfering the beam.

\subsection{Engineering Applcations}

We can use the calibrated QD to better study the nature of the dynamic pointing errors on the GBT and how they affect science observations. There are three conditions on the telescope under which dynamic pointing errors are the dominant limit on sensitivity. The first case is when a resonance in the servo control system is active, causing an oscillation in the tracking error, the second case is when the feedarm's structural resonance is excited, and the third case is when the feedarm is buffeted by winds. The QD effectively detects most of the pointing errors in the latter two cases. Figure 2.9 shows the Fourier transforms of pointing data from half-power tracks, the calibrated QD data, and the servo following error. Of these errors, the ones due to wind are the most interesting, as they are the largest and constrain high-frequency observing on the GBT. Prior to the use of the QD, it was often assumed that windinduced pointing errors were due to excitation of the feedarm structural resonance (see e.g. Wells 2000) and would be correlated with wind speed. Both of these as-

sumptions, however, are challenged by the actual QD data. One can see in Figure 2.9 that the primary structural resonances of the feedarm at 0.6 and $0.8 \mathrm{~Hz}$ are barely visible above the continuum on a windy day. Instead, wind-induced pointing errors take place on a timescale of minutes. Figure 2.10 shows the comparison of the QD data and two windy half-power tracks in the time domain, demonstrating that our calibration is reasonably accurate.

Smith et al. (2000) performed similar measurements under windy conditions on the Nobeyama $45 \mathrm{~m}$ telescope. Rather than half-power tracks, they used lunar limb scans to measure astronomical pointing, which serve similar purposes, but eliminate 
any directional ambiguity. They found a similar result in that their largest pointing errors were from "quasi-static" motions (i.e. those with a frequency lower than 0.1 $\mathrm{Hz}$ ). However, they also found several structural resonances in their pointing data that were still significant and claimed that these resonances were excited by the wind. Figure 2.11 shows one of their lunar limb scans and two of our azimuth half-power tracks, including one under comparable conditions $\left(\approx 4 \mathrm{~m} \mathrm{~s}^{-1}\right.$ winds $)$ and one under calm conditions with similar smoothing applied to the data. It is readily apparent in our data that the structural resonances are not excited at all by the wind. In frequency space, the pointing behavior of the GBT above $0.3 \mathrm{~Hz}$ seems to be the same regardless of the wind. The presence of wind simply raises magnitude of the the low frequency $(<0.3 \mathrm{~Hz})$ pointing errors. With the addition of QD data, we can say for certain that the low frequency errors we observe are due to the motion of the feedarm. Additionally, our data extends over 20 and 33 minutes, rather than a few minutes for the Nobeyama data. We continue to detect wind-induced low frequency pointing errors at even lower frequencies than were measured in the previous work, although the spectrum flattens below $0.01 \mathrm{~Hz}$. This flattening is consistent with the typical spectral distribution of wind disturbances at 100m above the ground (Simiu and Scanlan 1996, p. 61), but different from our $25 \mathrm{~m}$ elevation observed wind spectrum, which follows a $\nu^{-1}$ power law and turns over at a frequency of about $0.001 \mathrm{~Hz}$.

Various attempts to compare wind speed to QD-measured feedarm deflection have not shown an obvious correlation, although RMS QD pointing errors vary roughly with $v_{\text {wind }}^{2}$. Instead, we have discovered that the direction of the wind is actually the most relevant quantity. On a windy day, we locked the telescope elevation axis at the survival elevation of 60 degrees, which is the position where the dish is closest to horizontal, and rotated the telescope $360^{\circ}$ about the azimuth axis in each direction. 
The QD-inferred pointing error versus the wind direction relative to the telescope pointing direction (accounting for both wind shifts and the ongoing rotation of the telescope) is shown in Figure 2.12. The physical mechanism for this response is that the wind is blowing the feedarm forward along the wind vector, which results in a mostly sinusoidal signature in both elevation and cross-elevation pointing error, with a phase difference of $90^{\circ}$. The feedarm response is twice as large in the crosselevation direction as it is in the elevation direction. We suspect that this effect is due to reduced structural stiffness in cross elevation rather a change in drag coefficient since the drag coefficient of a lattice structure such as the feedarm only varies by about $10 \%$ with changes in angle of attack (Simiu and Scanlan 1996, p. 440). The telescope dish can also provide a shielding effect when the wind is blowing from the front or back of the dish, and this effect has been observed in the aforementioned serendipitous dataset. However, since the telescope was locked at survival elevation during the rotation experiment, any shielding effect should be minimized. This result provides important constraints on the common use of the offset pointing technique (in which the local pointing corrections are periodically measured by pointing on a quasar near on the sky to the science target (Condon and Yin 2001)). Clearly, under windy conditions, it is more important to choose a pointing calibrator nearby in azimuth rather than elevation to minimize this source of error due to the change in the projected angle to the wind force vector. From these data, the pointing change incurred by a worst case $180^{\circ}$ slew can be as large as $50^{\prime \prime}$ in $6 \mathrm{~m} \mathrm{~s}^{-1}$ of wind.

The cross-elevation direction component of the QD calibration was determined astronomically, so it could represent pointing errors from sources other than just the feedarm, such as coupled motion of the main dish. In order to discriminate between the motion of the feedarm and the motion of the telescope as a whole, we used 
three three-axis accelerometers on the telescope: one on the ceiling of the Gregorian receiver cabin near the illuminator (accelerometer 1), one at the edge of the dish nearest the feedarm (accelerometer 4), and one immediately adjacent to the QD detector (accelerometer 5). Figure 2.1 shows these locations. The accelerometer data were processed to remove the signal of gravitational deflection in a manner similar to that used for removing the signal from the QD data since they are all located on the tipping structure. We then compared the magnitude of the accelerations at these three accelerometers under windy conditions and under deliberate feedarm excitation. The result was the same in both cases: motion at accelerometer 1 was much greater than motion at accelerometer 4, which was greater than motion at accelerometer 5 . In other words, the telescope pointing errors are almost entirely due to the motion of the feedarm alone (represented by accelerometer 1), rather than the coupled motion of the dish (represented by accelerometers 4 and 5 ).

\subsection{Astronomical use with MUSTANG}

MUSTANG (MUltiplexed Squid Transition-edge sensor Array at Ninety Gigahertz) is an imaging bolometer array used on the GBT at $3.3 \mathrm{~mm} / 90 \mathrm{GHz}$ (Dicker et al. 2008). It is currently the highest frequency instrument in use on the GBT. As such, it is the instrument that would benefit the most from pointing improvements. MUSTANG's primary scan pattern is not a simple stare at a source, but rather a daisy petal, billiard ball, or other moving scan. As a result, the data reduction pipeline for MUSTANG must use the timestream of the position of the telescope as a critical input. Therefore, there is little need to correct for static or dynamic pointing errors in real time. So long as the errors are recorded and smaller than the field of view of the array $\left(30^{\prime \prime}\right)$, they can be corrected during the data reduction stage. 
Such corrections have already been implemented into the MUSTANG data reduction software written in IDL. By default, the MUSTANG pipeline takes QD data recorded during each scan, subtracts a median from the QD data from that scan in order to remove drift in the $\mathrm{QD}$, then removes the QD-inferred pointing errors from the data stream. More advanced users can choose to compute a median that is derived and used over several scans. As a result, the influence of the wind can be nearly completely removed. Figure 2.13 shows the improvements the QD can make over the course of 20 minutes integration time on a windy night. The primary effect of wind is to smear out the source in the cross-elevation direction. When used properly, the QD can remove a majority of the detrimental effects of observing under windy conditions.

A precise figure is difficult to calculate since this experiment was done on a bright quasar on a windy night. Comparable data from a calm observing run is not available for direct comparison due to quasar variability, telescope efficiency variability, and other factors. We assume that point source sensitivity goes as $\frac{P}{W^{2}}$, where $P$ is the peak of the Gaussian point-spread function (PSF) and $W$ is the width of the Gaussian PSF (i.e. proportional to flux density on the sky, brightness per unit area). We do not know the true peak of the source, however. We calculated an ideal peak based on the peak value required to make a Gaussian with the same total integrated flux of the windy Gaussian, but with an ideal FHWM, which is more predictable. The range given here reflects different assumptions in the calm PSF, with 9 arcseconds being the absolute best and 9.3 arcseconds representing a more typical PSF observed under calm conditions. In wind speeds of $5 \mathrm{~m} / \mathrm{s}$, we go from an uncorrected point source sensitivity of $47-53 \%$ to a corrected sensitivity of $78-88 \%$ compared to our assumed benign-weather sensitivity, for a recovery of $58-74 \%$ of the sensitivity lost to wind. Several scientific papers have been published using MUSTANG data processed with 
the QD correction as the authors have found that it improves the consistency of the results (Cotton et al. 2009; Dicker et al. 2009; Mason et al. 2010; Korngut et al. 2010; Shirley et al. 2010).

\subsection{Other Astronomical Uses}

The QD can benefit observers using GBT instruments other than MUSTANG as well. One future project is to incorporate a flag in the observing data whenever the beam has moved significantly off-source according to the QD. Users can then either delete these time periods or attempt to correct the measured signal during these time periods. Either method will improve the accuracy and consistency of the measurement even though the loss in signal-to-noise ratio cannot be recovered. However, the ideal method of QD application would be to somehow apply corrections in real time while observing, to prevent a loss of sensitivity in the first place. The subreflector servo system on the GBT provides this capability. Recently, we performed an experiment of taking real QD data recorded under windy conditions and feeding it into the subreflector servo during a half-power track in calm conditions to demonstrate that the servo can follow input from the QD. The resulting data shown in Figure 2.14 indicate that the telescope pointing was perturbed exactly as expected. Thus, it is clear that the subreflector is capable of following realistic data from the QD. Since the bandwidth of the QD correction is only a few $\mathrm{Hz}$, there is sufficient time (i.e. many milliseconds) to perform the small amount of signal processing and update accordingly the subreflector trajectory. The only remaining task is to connect the real-time data path in the GBT M\&C system, which we expect to attempt in the near future. After a suitable testing period, real-time corrections with the subreflector will made available as an option for high-frequency observers, thus eliminating the 
need for any corrections in the post processing if enabled.

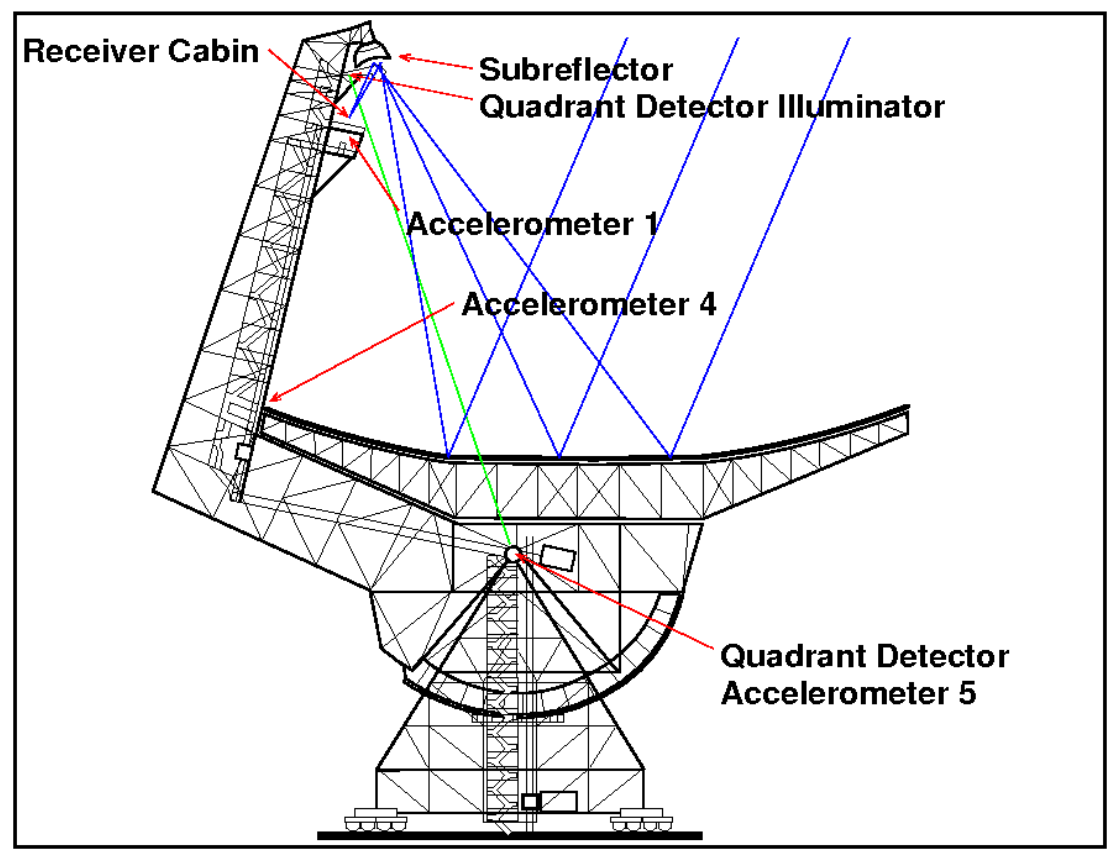

Figure 2.1: Layout of the GBT shown in profile observing at an elevation of $66^{\circ}$. Dotted lines show the approximate optical path of the telescope for high-frequency instruments. The dashed line shows the optical path between the illuminator and the quadrant detector. 

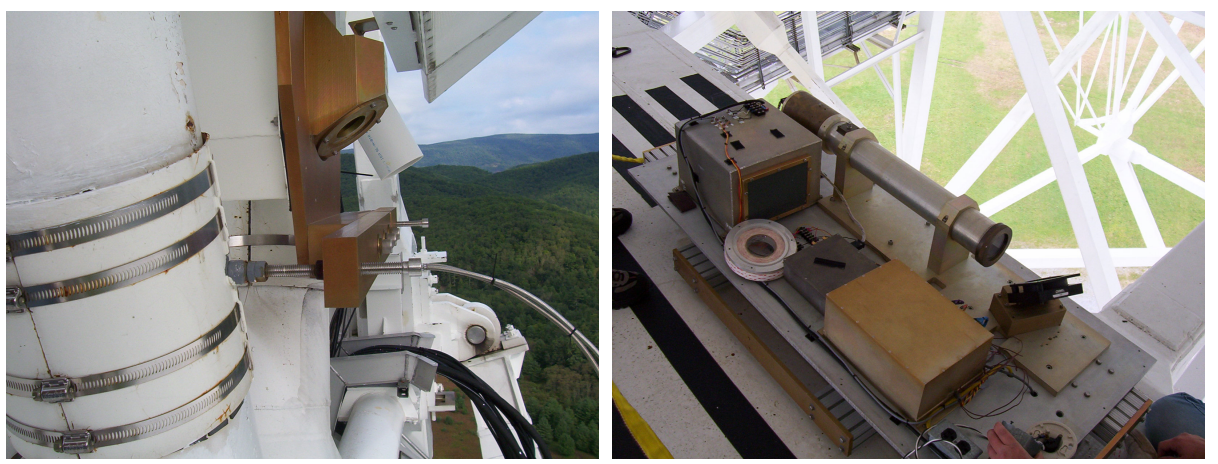

Figure 2.2: Left panel) Photo of the QD illuminator assembly (gold anodized housing) located just below the subreflector; right panel) Photo of the QD telescope tube, mirror, and electronics with the weather cover removed. It is located above the elevation axle and about $15 \mathrm{~m}$ under the primary dish.

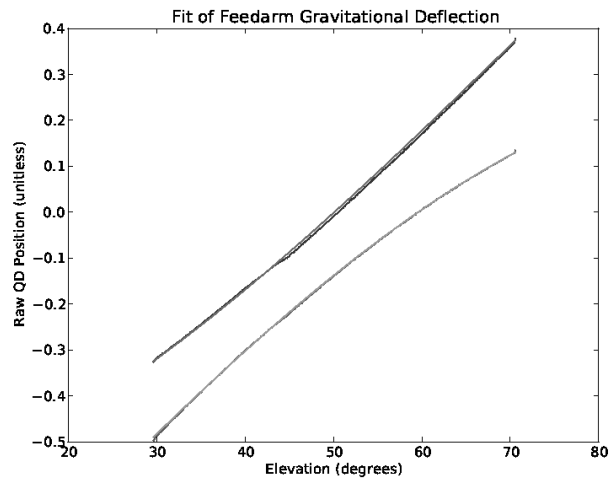

Figure 2.3: Signal of the feedarm deformation (blue and green) due to the change in the gravity vector at different elevations, along with the model fit (red and cyan). This deformation is already accounted for in the telescope pointing model, so it must be removed from the QD signal in order to isolate the residual dynamic pointing errors that it also measures. 


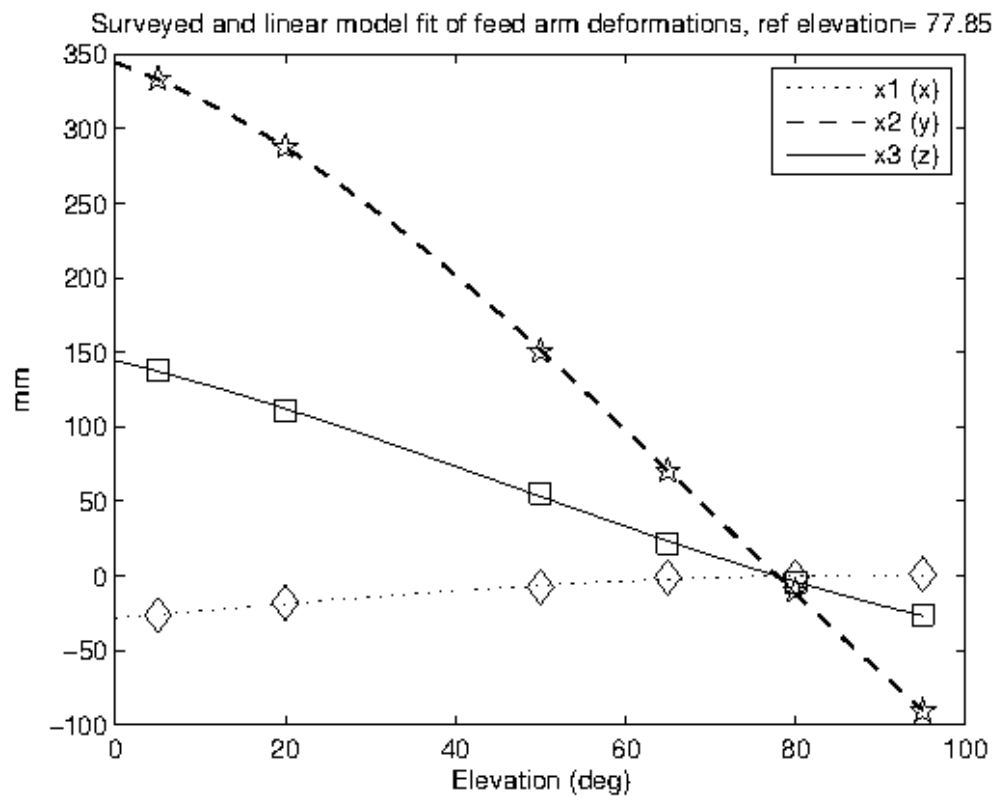

Figure 2.4: The relative deflection of the GBT feedarm vs. elevation as measured by traditional surveying techniques. The points are the measurements and the lines are the best fits.
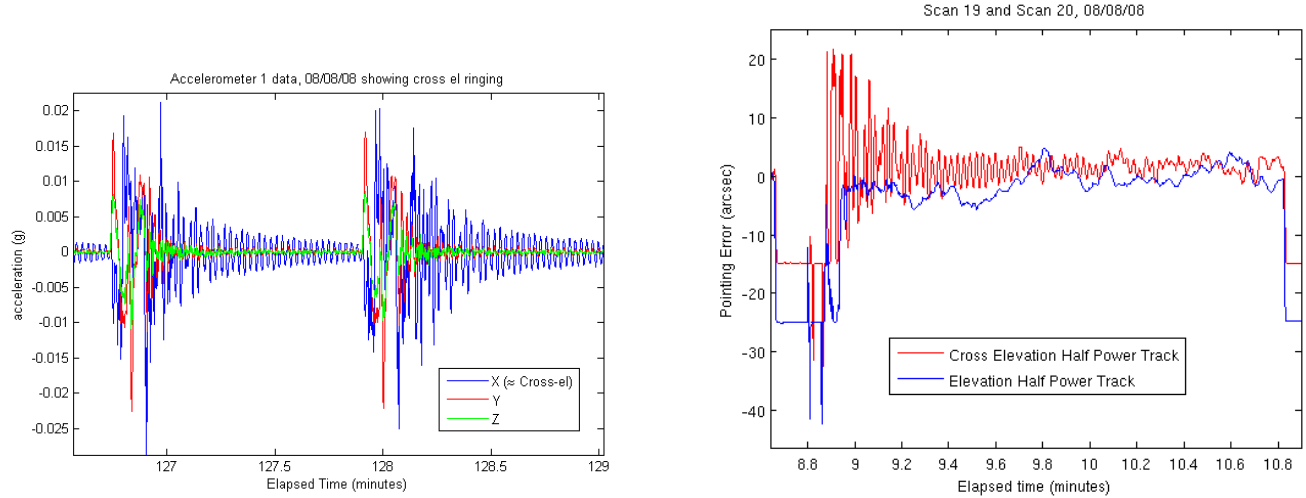

Figure 2.5: Data from both the three-axis accelerometer (left) in units of g-force and from half-power tracks (right) demonstrating that feedarm oscillation occurs almost entirely in the cross-elevation direction. 


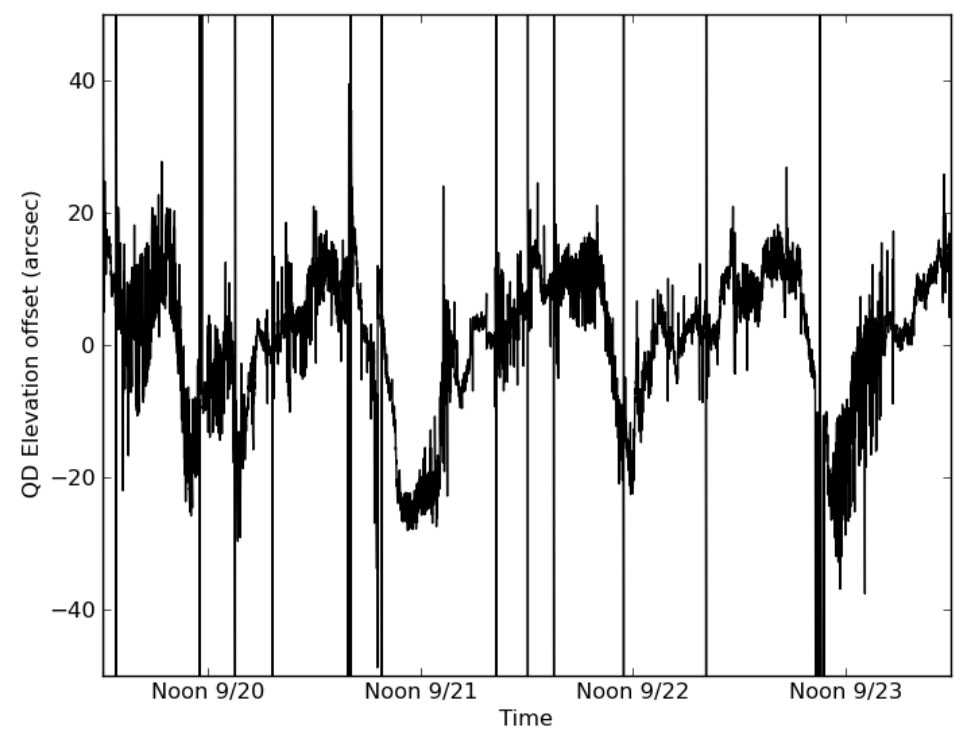

Figure 2.6: Thermal influence on the QD signal. This plot shows the change in QD elevation reading vs. local time in September 2010. A clear diurnal signal can be seen, whereas the signal is fairly stable at night. The steepest slope is of order a few arcseconds per hour. The occasional large excursions are due to the brief blockages of the beam by prime focus boom as it deploys or retracts between observing sessions. 

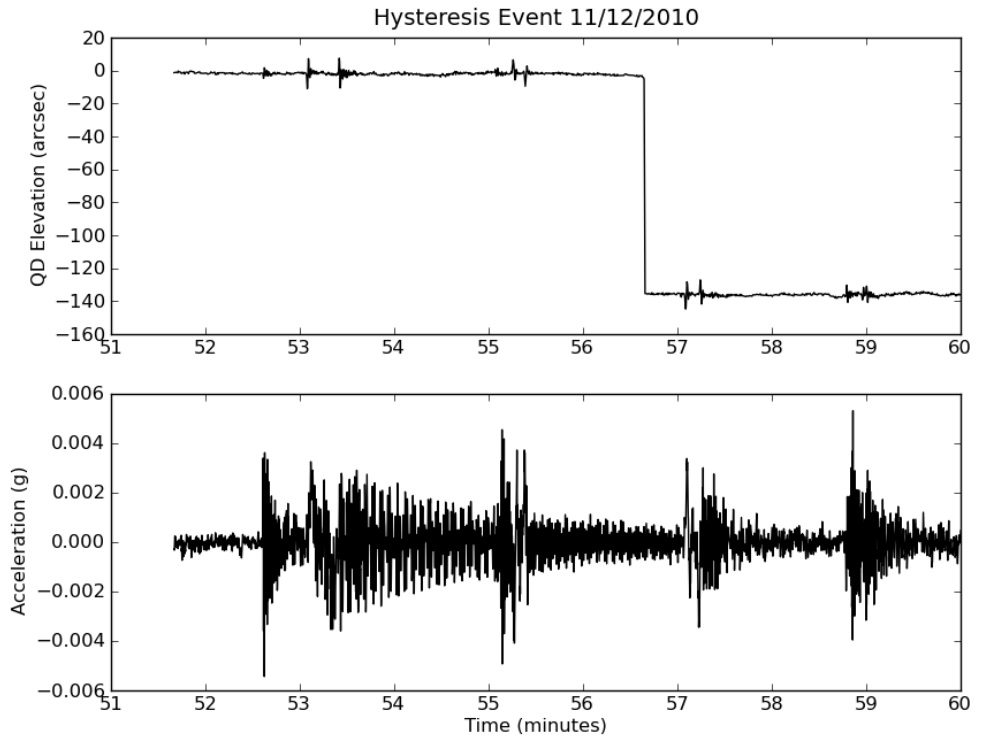

Figure 2.7: Top panel) An "hysteresis event" in detail in which the QD reading changes by $120^{\prime \prime}$. Bottom panel) The event in the QD does not show up in the feedarm accelerometer, while several real feedarm ringing events of much smaller magnitude do. Thus, the hysteresis event must be in the QD electronics or optics, rather than the telescope structure. Both the QD and accelerometer data have been corrected for the expected changes in the gravity vector. 


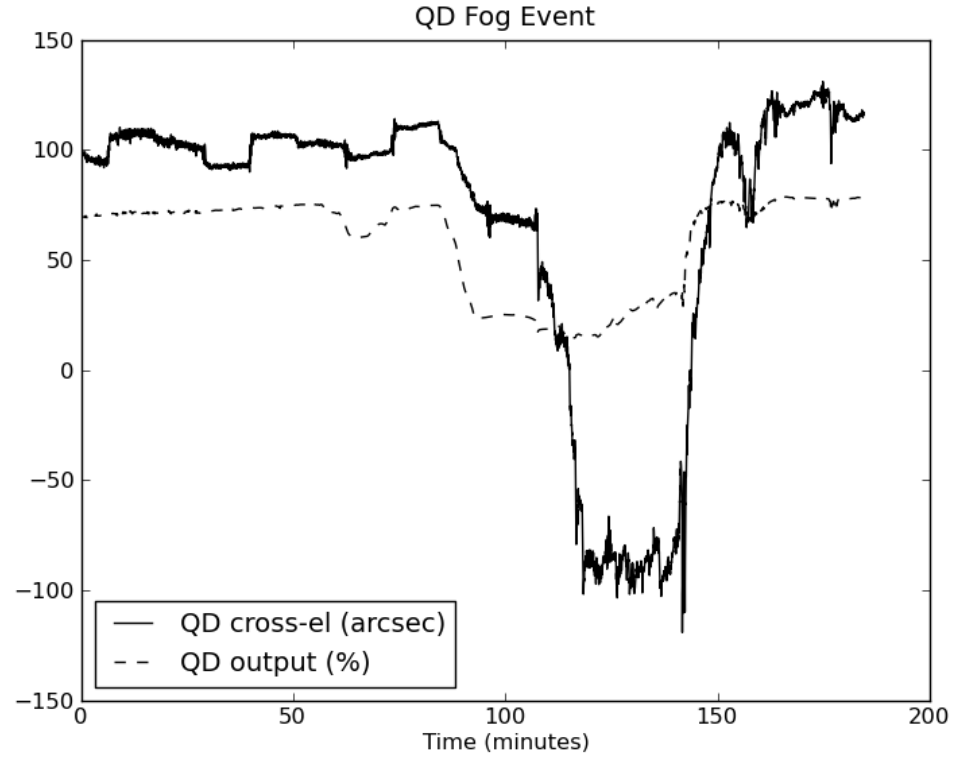

Figure 2.8: A plot of the output voltage from the QD's PSD (in percent of the A/D full scale) and the cross elevation correction signal vs. time during a fog or precipitation effect. The effects of snow, rain, fog, and snow accumulation are indistinguishable. The effect on the signal begins at around $t=85$ minutes, and continues until $t=160$ minutes. 

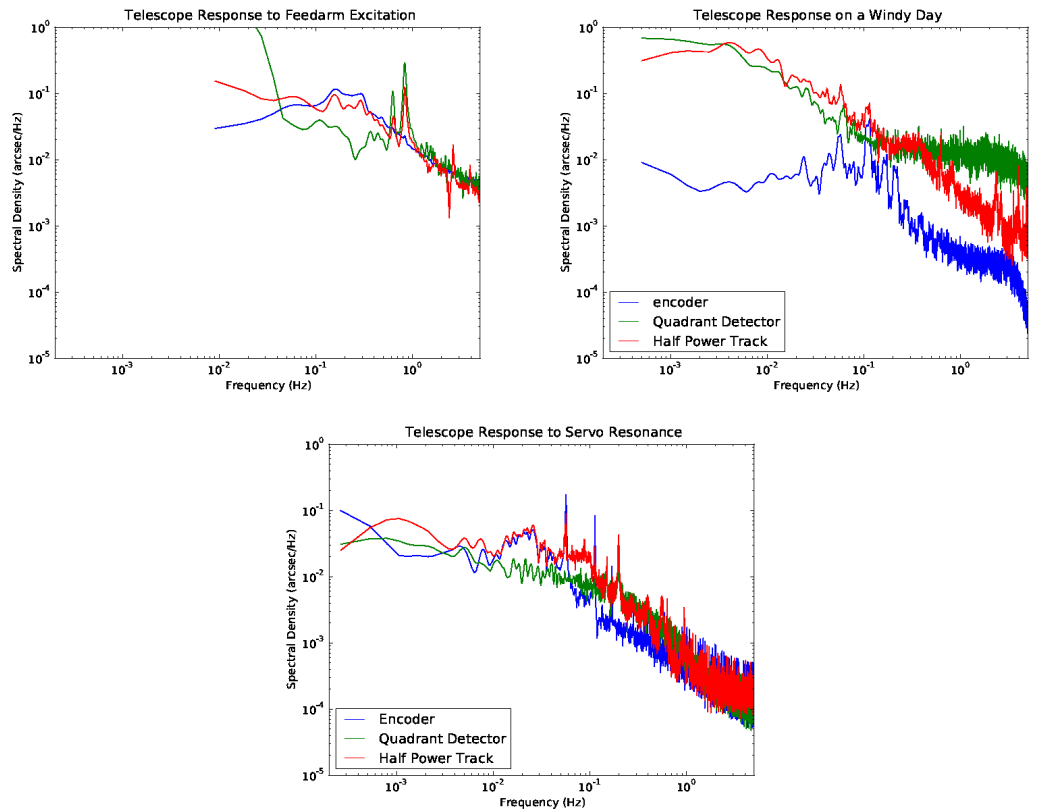

Figure 2.9: Frequency-space diagrams showing the magnitude of the observed pointing errors (inferred from the receiver half-power track data, the QD, and the encoder) vs. frequency under various different conditions: upper left) feedarm resonance; upper right) windy conditions; bottom) servo resonance.
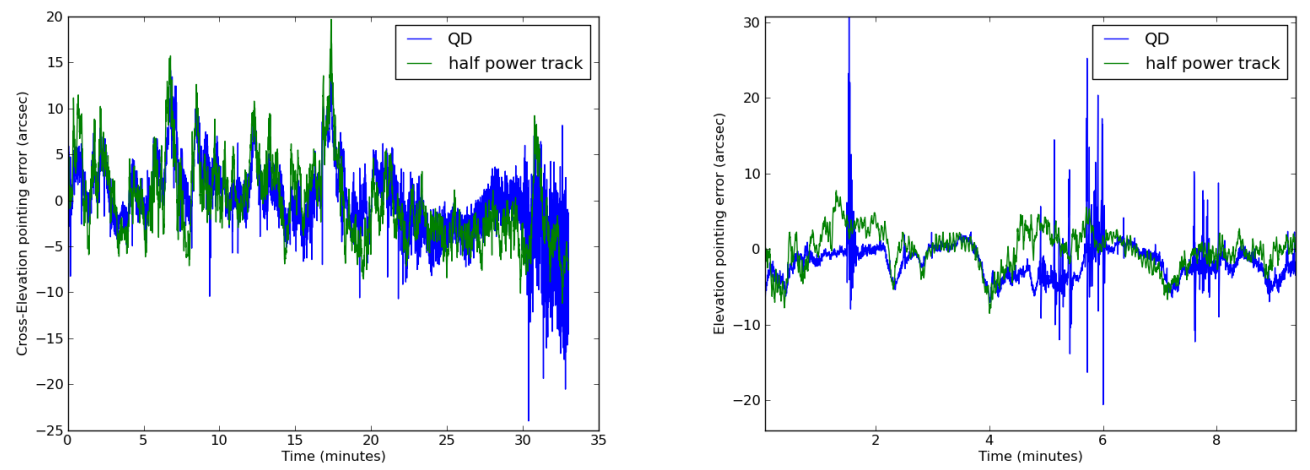

Figure 2.10: Plot comparing the QD-inferred pointing error and half-power track pointing errors in the time domain. The average wind speed during these observations was $3 \mathrm{~m} \mathrm{~s}^{-1}$. Note that the cross-elevation errors are larger and also yield a much better fit to the QD data. 

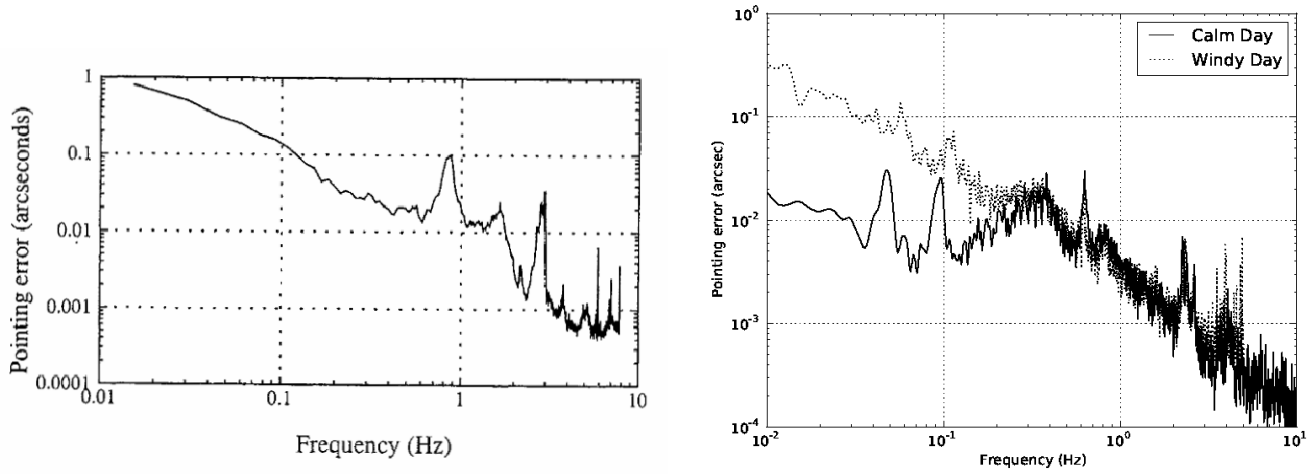

Figure 2.11: Comparison of the Fourier transform of pointing errors between the Nobeyama $45 \mathrm{~m}$ telescope from Smith et al. (2000) (left) and the GBT at Ka band (right). The Nobeyama data and our windy data were both taken at wind speeds of $\approx 4 \mathrm{~m} \mathrm{~s}^{-1}$. Our calm data comes from a day when wind speeds were $<1 \mathrm{~m} \mathrm{~s}^{-1}$. There is no difference between the GBT structural resonances $(0.6,0.8$, and $2.1 \mathrm{~Hz}$ ) on the calm day (February 28, 2008) and the windy day (January 24, 2008). In fact, the only difference is the presence of more "continuum" power at frequencies under $0.3 \mathrm{~Hz}$. The features at 0.046 and $0.093 \mathrm{~Hz}$ seen in the calm day's data are due to the known servo resonance and correspond to the azimuth motor axle rotation rate and its harmonic. The exact frequency of these features depends linearly on the tracking velocity. On the calm day, the scan length was 20 minutes with an average tracking rate of $3.69^{\prime \prime} \mathrm{sec}^{-1}$ while on the windy day the scan length was 33 minutes with an average rate of $4.34^{\prime \prime} \mathrm{sec}^{-1}$. Thus, the spectral features appear at slightly higher frequencies in the windy day's data, but their relative contribution to pointing error compared to the continuum is less. 

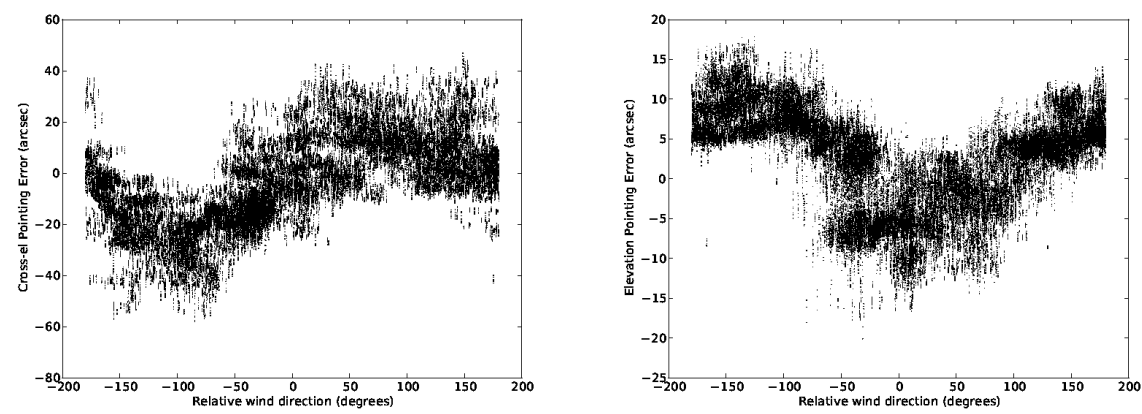

Figure 2.12: Plots showing QD response to a change in wind direction induced by rotating the telescope $360^{\circ}$ clockwise and counterclockwise on 30 March 2010. The average wind speed during each rotation is 6-7 $\mathrm{m} \mathrm{s}^{-1}$. The wind direction in the above plots represents measured wind direction minus telescope azimuth. In spite of substantial fluctuations in wind direction during the hours of these rotations, the pattern holds true.

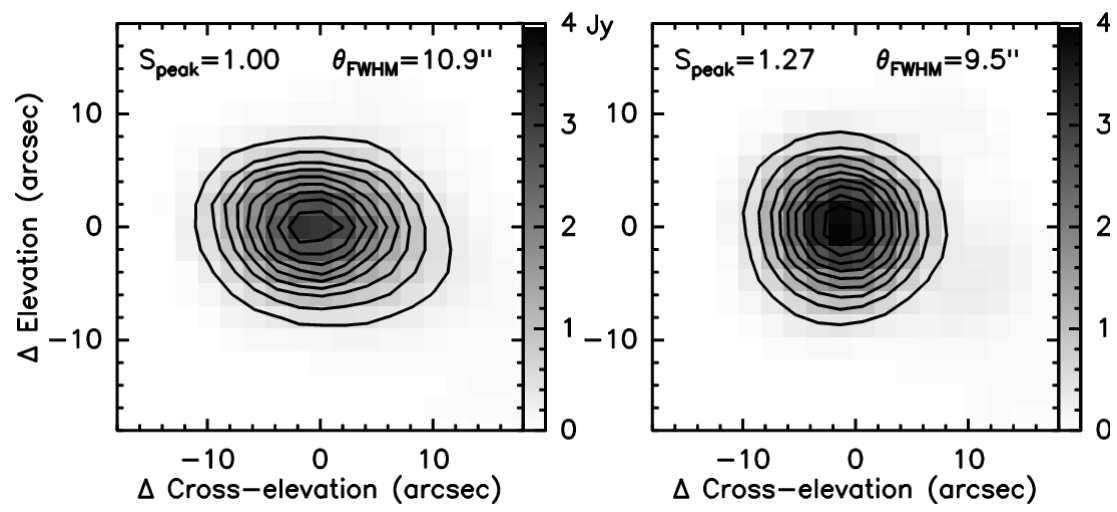

Figure 2.13: MUSTANG images of a bright quasar obtained on a windy $\left(\approx 6 \mathrm{~m} \mathrm{~s}^{-1}\right)$ night combining several scans with a total integration time of about 20 minutes from March 4, 2010. The image on the left has had no QD correction applied. The image on the right has had a QD correction applied using one median value to remove QD drift for all of the scans. For reference, the typical size of a MUSTANG beam under ideal conditions is $9.0^{\prime \prime}$. 

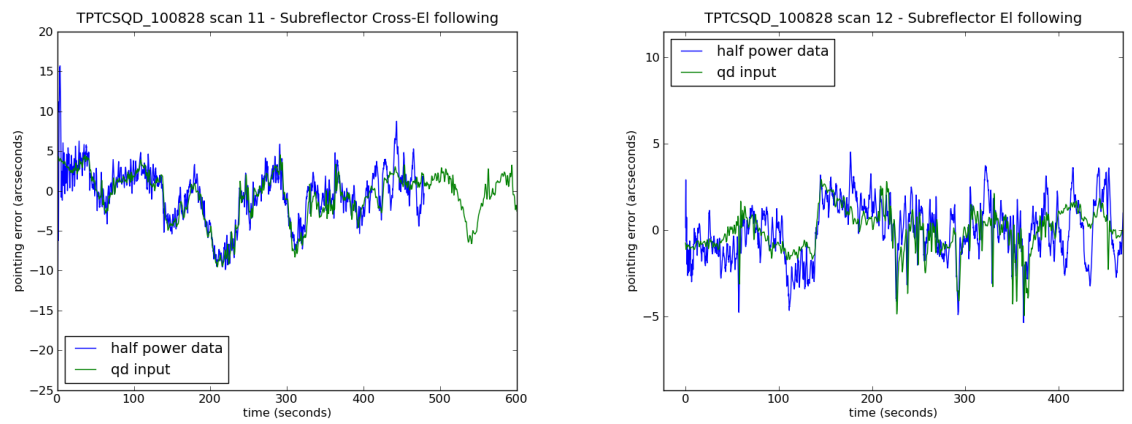

Figure 2.14: An experiment demonstrating the potential usefulness of the QD to correct pointing in real-time. The QD signal from a windy dataset is used as an input to the subreflector on a calm day. The resulting pointing of the telescope is then measured during a half-power track on a quasar. Left panel) a cross-elevation halfpower track. Right panel) an elevation half-power track. Both axes of the subreflector are easily able to follow the real QD data as an input trajectory and produce the expected pointing error. 


\section{Chapter 3}

\section{Using the QD with science observations}

\subsection{Iapetus Science Data and Wind}

How can the quadrant detector be used with actual science observations? This chapter will consist of an attempt to use the QD to search for wind-induced pointing errors during real science observations, namely the ones which are the main subject of this thesis. The main description of my Iapetus observations such as dates and observing techniques can be found in a subsequent chapter, as this chapter will deal solely with wind-induced ponting errors.

Table 3.1 lists the range of wind speeds during my Iapetus observing runs. In general average winds less than about $4 \mathrm{~m} \mathrm{~s}^{-1}$ over a 20 minute period tend to have a relatively minor effect on pointing. Only 3 of the 13 runs show winds greater than $4 \mathrm{~m} \mathrm{~s}^{-1}$ at all, which is good from a science perspective (no wind pointing errors most of the time!), but bad from the perspective of trying to demonstrate the QD's usefulness (no wind pointing errors to test against). Nonetheless, QD data for the 


\begin{tabular}{|l|c|}
\hline run \# & wind speed $\left(\mathrm{m} \mathrm{s}^{-1}\right)$ \\
\hline 00 & $3-4$ \\
01 & $1-2$ \\
02 & $3-4$ \\
03 & 0 \\
04 & 0 \\
05 & $3-6$ \\
06 & $1-2$ \\
07 & $1-3$ \\
08 & $1-3$ \\
09 & $3-5$ \\
10 & $1-4$ \\
11 & $1-5$ \\
12 & $2-3$ \\
\hline
\end{tabular}

Table 3.1: Wind speeds during Iapetus observing sessions from weather station 1.

two windiest runs will be examined to look for wind-induced pointing errors.

\subsubsection{Ka-band Observations}

The ninth run (referred to as run G2 in the observing chapters) is a critical run in terms of its scientific utility, as it provided conclusive proof that Iapetus has strange behavior in the $1 \mathrm{~cm}$ region. It also happened to have one of the higher amounts of wind of any of the observing runs. Could the wind have played a role in the derived measurements? The answer is an unequivocal "no" thanks to the quadrant detector. Figure 3.1 shows the quadrant detector readings during the science portion of the observing run. The rms pointing errors derived from the QD during the entirety of the science part of the observing run is 1 arcsec for cross-elevation and about 2 arcsec for elevation. However, the elevation pointing errors are likely much smaller (less than 1 arcsec rms) since all of the major variation in elevation is due to a slow drift. In this case, the slow drift is likely an example of the diurnal thermal variation depicted in the previous chapter, rather than any ill effect due to wind, particularly 
in light of the fact that the observation took place around local noon. The beam size of the GBT at $1 \mathrm{~cm}$ is about 25 arcsec, so pointing errors of order an arcsecond are negligible $(<0.5 \%)$.
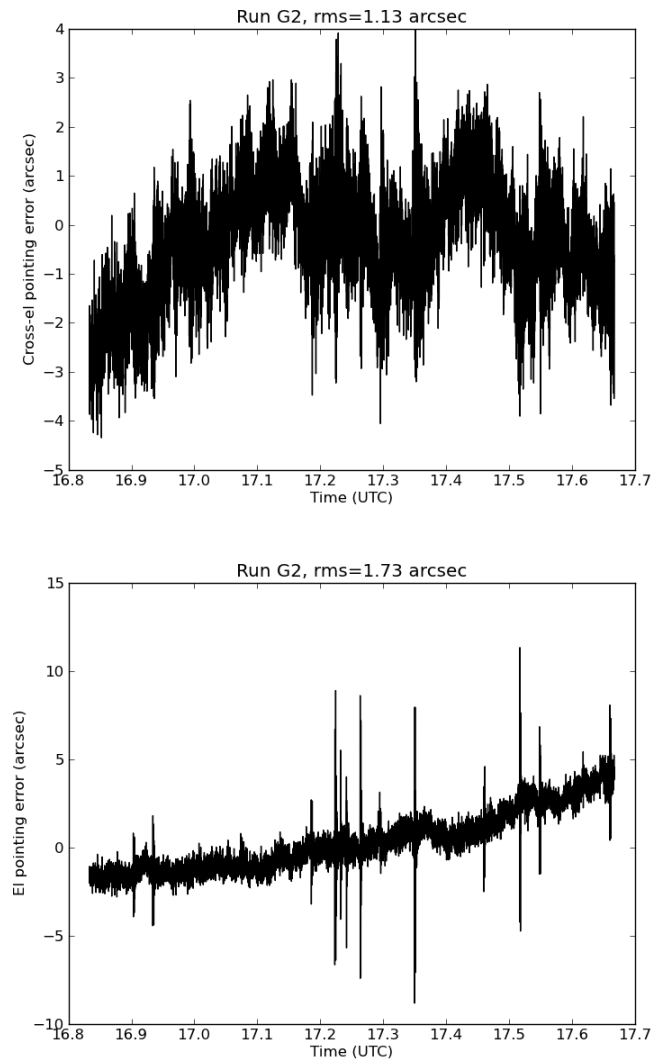

Figure 3.1: Pointing errors in cross-elevation (left) and elevation (right) as inferred from the quadrant detector instrument during one of the important CCB science observing runs on $11 / 19 / 2011$, which also happened to be one of the windiest Kaband runs.

\subsubsection{MUSTANG Observations}

The fifth run was a MUSTANG observation (referred to as run Cm in the observing chapters). The winds were also reasonably strong during the observing run. Did the 
winds have a substantial effect on the observations? The answer is also an unequivocal "no." This time the most compelling proof comes not from the QD, but from observations of Iapetus itself. Every MUSTANG observation creates a map of the target object and also allows for measurement of the beam shape, which degrades in the presence of wind. The beamwidth for the Iapetus observation over the entire observing run estimated by fitting a gaussian to the source is about $9.5 \operatorname{arcsec}(9.8 \mathrm{in}$ cross-elevation and 9.0 in elevation), which is not far off the typical good beamwidth of about $9 \operatorname{arcsec}^{1}$. In Figure 3.2, the QD shows only a gradual drift $(\approx 5 \operatorname{arcsec} / \mathrm{hr})$ over the duration of the observing run in cross-elevation, and negligible pointing errors in elevation. Quadrant detector corrections were used during the data reduction with a median computed on an a scan-by-scan basis, which substantially reduces the effect of structural resonance observed at the start of each scan in cross-elevation.

\subsection{Additional Science Data}

While the Iapetus data did not provide any opportunity to use the QD with the Kaband and definitively windy data, some subsequent observations for another project (AGBT12A_381) did. In February and March of 2012, data were acquired for a new project observing a multitude of solar system objects with the Ka-band receiver with the CCB backend. For the final run of the observing program on March 10, 2012, the winds ranged from 3-8 $\mathrm{m} \mathrm{s}^{-1}$ with variable direction but generally out of the north. In the case of this observing run, pointing errors were significant and predictable using the quadrant detector. Figure 3.4 shows the cross-elevation and elevation pointing errors inferred from the QD and those calculated during the AutoPeak pointing scans

\footnotetext{
${ }^{1}$ The diffraction limit is about 8 arcseconds, but this is not reached in practice. See, for instance, http://www.gb.nrao.edu/mustang/ for the 9 arcsec figure.
} 

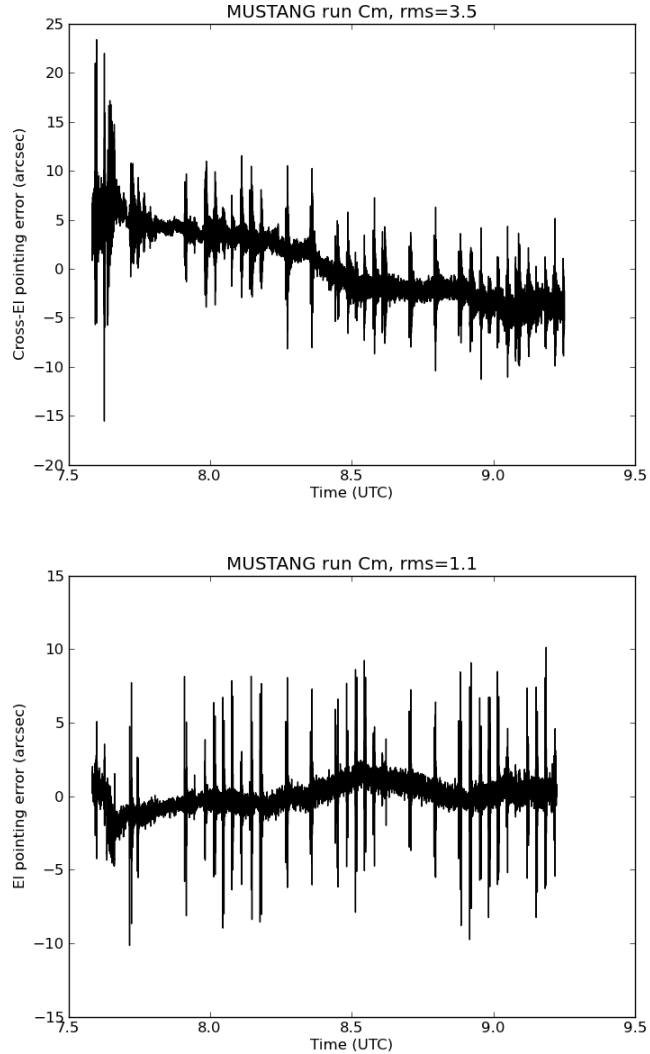

Figure 3.2: Pointing errors in cross-elevation (left) and elevation (right) as inferred from the quadrant detector instrument during the windiest MUSTANG observing run on $02 / 16 / 2012$. Note that the rms in cross-elevation is only 1.4 arcsec once the data are detrended. Spikes in the data are feedarm resonance during daisy scans. 
performed during that run. It was obvious during the original observing session that pointing was a problem, although not necessarily that it was being caused by the wind. Since a large number of sources were observed in very different locations on the sky, pointing errors as a result of large slews could have also been responsible for the large pointing errors. However, the agreement of the QD cross-el observations with the observed pointing errors clearly indicates that wind was the dominant source of pointing errors for cross elevation (although not in elevation). The QD cross-elevation observations also show a similar pattern (see Fig. 3.3) with respect to relative wind direction as seen in the the previous chapter, although wind gusts seem to be more relevant during this run (i.e. significant changes in pointing offset at similar relative wind directions).

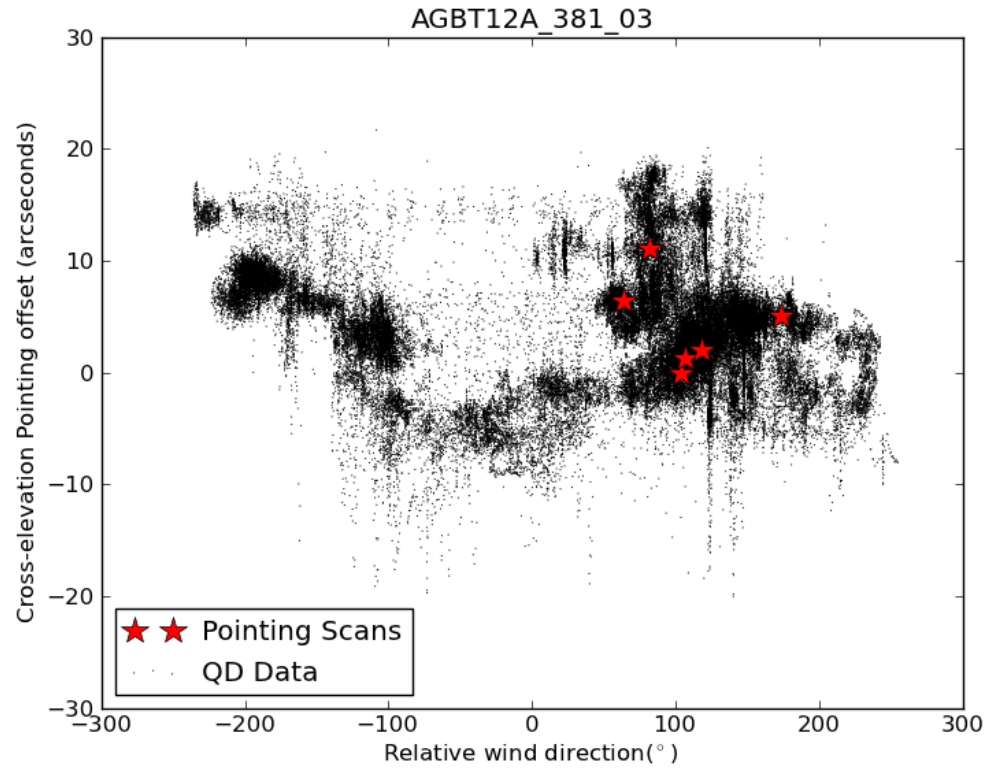

Figure 3.3: Pointing errors in cross-elevation vs. wind direction both from the QD and from the AutoPeak scans. The pattern seen here is similar to the sinusoidal pattern observed in the previous chapter. 
In a particularly interesting subset of this run, science measurements of a target source at approximately 19:45 showed that the source was only half as bright as expected in the shortest wavelength band. Table 3.2 shows the flux densities of the science source obtained before and after running an AutoPeak pointing scan. The QD cross-elevation reading indicated a pointing shift of about 10 arcsec since the previous AutoPeak pointing scan, which is substantial compared to the beam of 20 arcsec beam of the telescope at the shortest-wavelength band. Table 3.2 indicates that the decrement occurred exactly as would be expected in the other observing bands. After the next AutoPeak pointing scan (which made minimal change in the elevation local pointing correction and about a 10 arcsecond shift in the cross-elevation local pointing correction), the source flux was back to the expected value. The elevation QD results are not in great agreement with the AutoPeak pointing scans, but this result is not unexpected given that the elevation calibration is more tenuous. The result that both the QD and the elevation pointing coefficients mostly reflect a linear trend is an intriguing result that may merit further study.
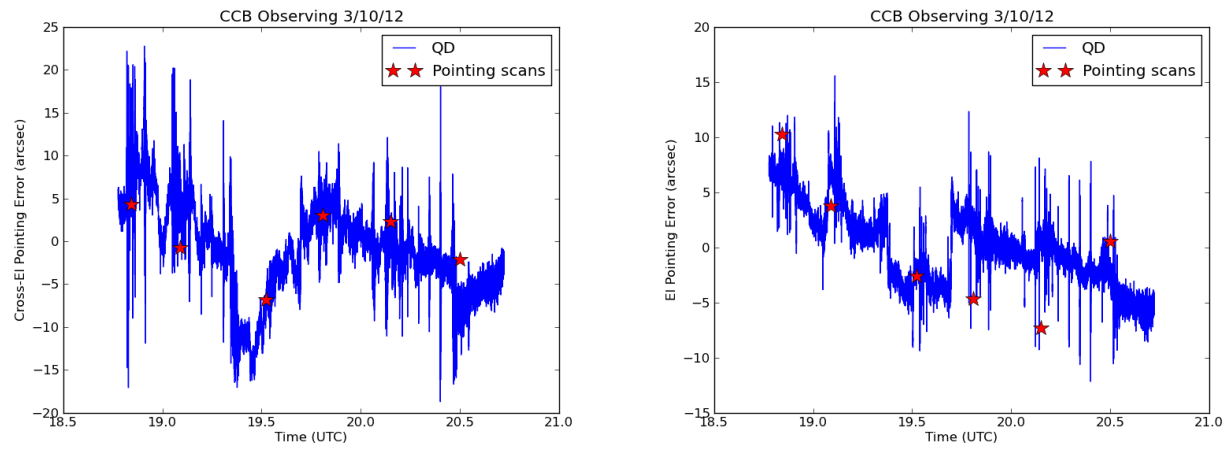

Figure 3.4: Pointing errors in cross-elevation (left) and elevation (right) as inferred from the quadrant detector instrument during a windy CCB observing run. The cross-elevation pointing errors measured by the QD seem to match up well with the observed AutoPeak procedure pointing offset results. 


\begin{tabular}{|l|c|c|c|c|}
\hline $\begin{array}{l}\lambda \\
(\mathrm{mm})\end{array}$ & $\begin{array}{c}\text { Before } \\
(\mathrm{mJy})\end{array}$ & $\begin{array}{c}\text { After } \\
(\mathrm{mJy})\end{array}$ & $\begin{array}{c}\text { \% decrement } \\
\text { (observed) }\end{array}$ & $\begin{array}{c}\text { \% decrement } \\
\text { (computed) }\end{array}$ \\
\hline 10.8 & 1.89 & 2.57 & 27 & 27 \\
9.6 & 2.42 & 3.69 & 36 & 33 \\
8.6 & 2.90 & 5.05 & 43 & 39 \\
7.8 & 3.57 & 6.40 & 44 & 45 \\
\hline
\end{tabular}

Table 3.2: Flux densities from two single nods on a science target (az: 205 el: 46) before and after an AutoPeak pointing scan (az:215 el:47). Decrements observed based on "after" fluxes and computed decrements based on a gaussian with FWHM of $740 / \nu(\mathrm{GHz})$ and an assumed pointing error of 9 arcsec derived from the QD data and AutoPeak pointing procedure.

In conclusion, the quadrant detector clearly indicates that wind-induced pointing errors are not a large influence on the thesis science results presented later in this thesis. However, during a non-thesis science observing run, the quadrant detector successfully predicted a drop in flux due to wind-induced pointing errors. In addition, the QD pointing results correspond well with cross-elevation AutoPeak pointing scans over the duration of an entire two-hour observing run, although somewhat poorly with elevation scans. In the future, the QD data stream may be used by the telescope control system to apply real-time cross-elevation corrections via the subreflector servo system. Such corrections would improve the effectiveness of observing sessions such as this one, thus expanding the amount of time available for high-frequency observations. Alternately, the QD may soon be used in the offline data reduction software (GBTIDL) to flag data when the pointing error is larger than some threshold appropriate to the beam size at the observing frequency. 


\section{Chapter 4}

\section{Observations}

\subsection{Data acquisition}

The science focus of this thesis is an observing program targeting Iapetus in order to obtain a rotational light-curve at $\mathrm{Ka}-\operatorname{Band}(1 \mathrm{~cm})$ and $\mathrm{W}$-band $(3.3 \mathrm{~mm})$ with the GBT that lasted from March 2010 to February 2012. The original plan was to acquire observations evenly spaced approximately every 45 degrees of longitude. A diagram of the desired observing geometry is shown in Figure 4.1. Longitude is defined in terms of degrees west of the sub-Saturn longitude on Iapetus when Saturn is directly between Iapetus and the Sun. The listing of all observations by longitude is given in Table 4.1 and chronologically in Table 4.2.

\subsubsection{Instrument description}

The Ka-band receiver uses a special backend called the Caltech Continuum Backend $(\mathrm{CCB})^{1}$ which is able to measure four continuum bands simultaneously: 26-29.5, 29.5$33,33-36.5$, and $36.5-40 \mathrm{GHz}$, with band centers of $27.75 \mathrm{GHz}(10.8 \mathrm{~mm}), 31.25 \mathrm{GHz}$

\footnotetext{
${ }^{1}$ http://www.astro.caltech.edu/ tjp/GBT/
} 
(9.6 mm), 34.75 GHz (8.6 mm), and 38.25 GHz (7.8 mm), respectively. The Ka-Band receiver has an unusual polarization design, with one beam linear horizontally polarized and the other beam linear vertically polarized. However, since beam-switching was used in all observations, all measurements should effectively be unpolarized, as any polarization would be averaged out. ${ }^{2}$ The Digital Continuum Receiver (DCR) backend (with $1.28 \mathrm{GHz}$ bandwidth) was used also used when observing at Ka-band for pointing and focusing scans.

MUSTANG is a W-band $8 \times 8$ bolometer array centered at $90 \mathrm{GHz}(3.3 \mathrm{~mm})$ with a bandwidth of $18 \mathrm{GHz}$ (Dicker et al. 2008). As a bolometer, MUSTANG only measures total flux, so it is unaffected by any polarization in the source.

\subsubsection{Observing Strategy}

With the Ka-band runs, we used three different techniques for observing. The primary observing method was with beam-switching nods (Conway et al. 1965). The Kaband receiver is a dual-beam receiver with a separation of 178.8 " between beams. Measurements were made by taking the difference between the signals from the two beams in order to remove atmospheric fluctuations. In a single nod, the telescope begins with the source in beam 1, integrates for 30 seconds, then moves beam 1 offsource and beam-2 on-source for about 30 seconds. The resulting change in difference between the two receivers is twice the strength of the source. Figure 4.2 shows an example of the resulting plot of difference in the difference between beams during a single nod. The second technique used was that of nutated nods. Nutated nods are like normal nods, but have some deliberate offsets introduced in order to detect and

\footnotetext{
${ }^{2}$ At least in the case of solar system observations, where the sum of the polarizations is conserved in various polarization mechanisms. Furthermore, for unresolved mostly spherical objects, any polarization present should average out(e.g. the polarization at one point on the edge of the disk should cancel with the point on the opposite side of the disk).
} 
compensate for any unknown pointing errors. Figure 4.3 shows the nod pattern and nutated nod pattern from real observations of 3C286. Instead of simply staring at the source, the telescope is slewed slightly off the source in three different directions for each beam. This movement results in changes in flux density which should be identical to each other if the telescope has negligible pointing errors. Two-dimensional scans across the source were also used, and consist of slewing the telescope in a rectangular pattern centered on the source.

Table 4.2 gives a chronological listing of all of the observations. The Ka-band observation strategy evolved somewhat over the course of this project. Originally the strategy was to make maps on the location of Iapetus, and then to make maps later when Iapetus was not present in order to remove sidelobe contamination. This strategy was abandoned after it became apparent in the first few observing runs that Iapetus was not detectable in a map of any appreciable size. The GBT sensitivity calculator indicated a $10 \sigma$ detection of a $1 \mathrm{mJy}$ source in the middle Ka-bands in less than two minutes of observing. However, the sensitivity was worse than expected. On most of our observing runs, we only had a 1-2 $\sigma$ detection for each individual two-minute measurement. By the fourth observing run, all observations were done as nods rather than two dimensional patterns which could be turned into maps. Starting at the sixth observing run, "null nods" were added to the observing runs ( described in more detail in a later section). Their usage is listed in Table 4.3.

For the Ka-band observations, pointing scans on bright quasars were periodically performed to ensure pointing accuracy. For this project, the initial pointing and focus scans were done on either 1256-0547 (3c279), which was very near to Saturn or $1310+3220$, which was near the primary calibrator 3c286. Subsequent pointing scans were done about every half-hour on either $1310+3220$ or Saturn itself. 
All MUSTANG observing techniques are various types of two-dimensional patterns which cross the source. The only type of scan pattern used for this project was a Lissajous daisy pattern (Dicker et al. 2008) with a diameter of 3 arcmin and a period of 15 seconds.

All of the runs were dynamically scheduled (O'Neil et al. 2009). The weather at the Green Bank site is frequently poor for high-frequency observing, which includes both Ka- and W-band observations. Observers are alerted 24 hours in advance when the weather forecast and schedule allow observations. This project added an additional complication in that there were specific time windows in which observations needed to occur in order to align with Iapetus' orbital phase. The time windows were defined using project-based blackout dates, which prevented the project from being scheduled more than 15 degrees away from the desired longitude or from being scheduled to reobserve a previously-observed longitude. As a result, the project took about 1.5 years to complete, as poor weather prevented observing during some orbital phases.

\subsection{Data Reduction}

\subsubsection{Flux Density Calibration}

For flux density calibration, the bright quasar 3C286 was observed on each night of the Ka-band observations. We performed nods and nutated nods on the calibrator. 3C286 was assumed to be a $1.9 \mathrm{Jy}$ source at $32 \mathrm{GHz}$ with a spectral index of $\nu^{-0.8}$ (Wright et al. 2009). 3C286 was observed in all four bands each night in order to obtain a conversion between system temperature and flux density. Flux densities for Iapetus were then converted into brightness temperatures of the source using the formulae outlined in Chapter 1 so that the measurements would be independent of 

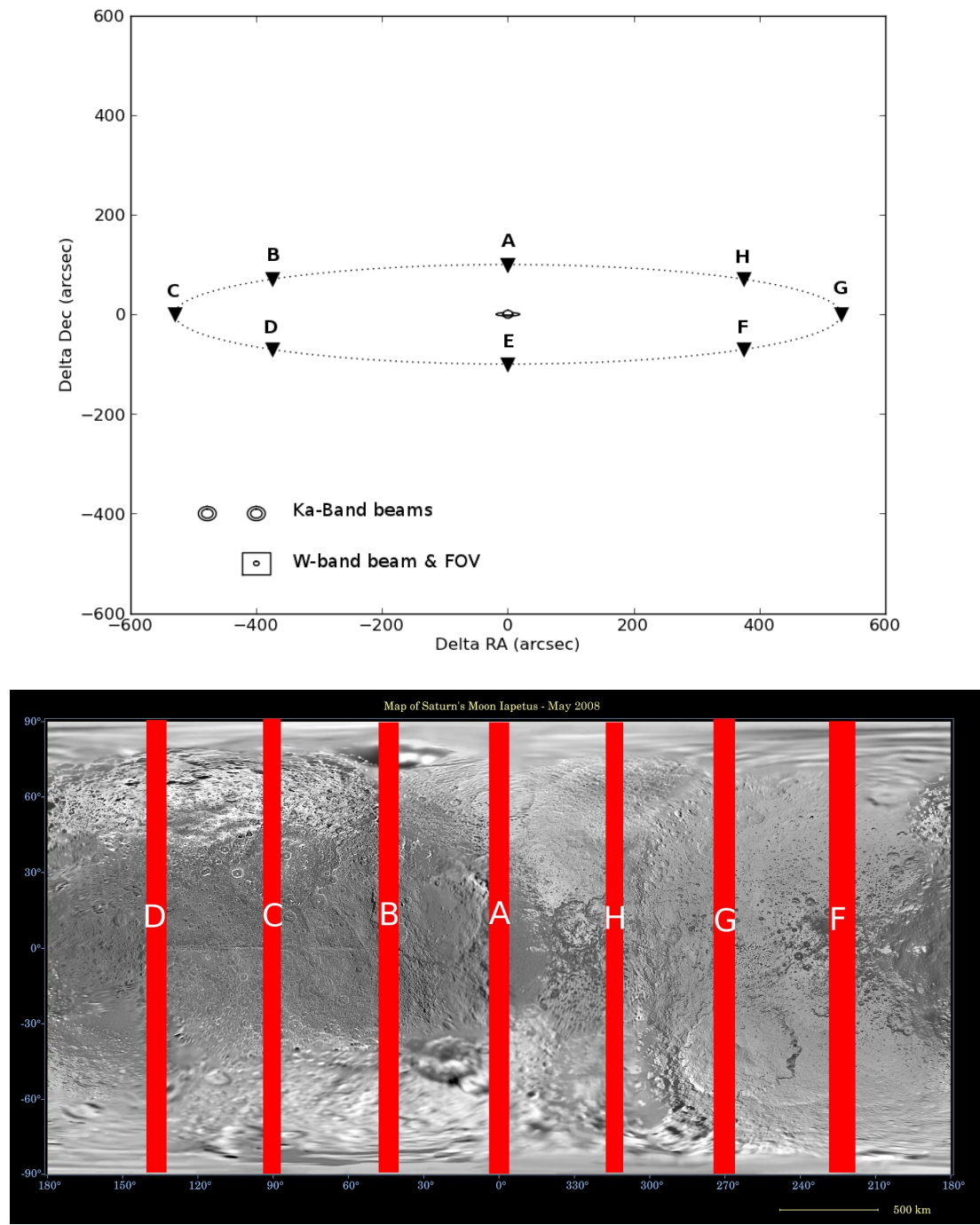

Figure 4.1: Layout of observations. A $\sim 0^{\circ}$ longitude, $\mathrm{B} \sim 45^{\circ}, \mathrm{C} \sim 90^{\circ}$, etc. Upper image shows layout in terms of Iapetus' orbit around Saturn. Lower images shows observations relative to Iapetus' terrain. The background image is NASA Public Image Archive \#11116. 


\begin{tabular}{|l|r|r|r|r|r|r|}
\hline Phase & Chron. Order & Ideal Lon. & Actual Lon. & Actual Lat. & r (AU) & $\Delta(\mathrm{AU})$ \\
\hline A & 1 & 0 & 1 & 12 & 9.59 & 10.42 \\
B & 2 & 45 & 41 & 13 & 9.59 & 10.33 \\
C & 7 & 90 & 103 & 12 & 9.63 & 8.76 \\
Cm & 5 & 90 & 99 & 13 & 9.59 & 8.93 \\
D1 & 3 & 135 & 125 & 13 & 9.56 & 10.06 \\
D2 & 8 & 135 & 140 & 14 & 9.65 & 10.64 \\
F1 & 6 & 225 & 218 & 13 & 9.59 & 8.66 \\
F2 & 11 & 225 & 226 & 15 & 9.69 & 9.45 \\
G1 & 4 & 270 & 282 & 13 & 9.60 & 9.53 \\
G2 & 9 & 270 & 265 & 15 & 9.68 & 10.50 \\
Gm & 0 & 315 & 326 & 10 & 9.51 & 8.58 \\
H1 & 10 & 315 & 322 & 15 & 9.70 & 10.39 \\
H2 & 12 & 315 & 316 & 15 & 9.73 & 9.18 \\
\hline
\end{tabular}

Table 4.1: List of various observing runs by longitude, as well as the distance of Iapetus from the Sun (r) and the distance from the Earth $(\Delta)$ at the time of the observation. Phases observed multiple times with the CCB are numbered. All observations are with the CCB instrument, except for those with the lower-case " $m$ " designating a MUSTANG observing run.

\begin{tabular}{|l|l|r|r|r|r|r|r|r|}
\hline Date & Phase & $\#$ & Band & $\Delta$ RA & $\Delta$ Dec & $\Delta$ X-El & $\Delta$ El & Total \\
\hline $2010 / 03 / 03$ & Gm $/ H m$ & 0 & $\mathrm{~W}$ & $\mathrm{x}$ & $\mathrm{x}$ & $\mathrm{x}$ & $\mathrm{x}$ & 300 \\
$2010 / 11 / 06$ & $\mathrm{~A}$ & 1 & $\mathrm{Ka}$ & 61 & 84 & -99 & 31 & 104 \\
$2010 / 11 / 15$ & $\mathrm{~B}$ & 2 & $\mathrm{Ka}$ & 343 & -5 & -264 & 217 & 343 \\
$2010 / 12 / 04$ & $\mathrm{D} 1$ & 3 & $\mathrm{Ka}$ & 359 & -142 & -258 & -286 & 386 \\
$2011 / 01 / 08$ & $\mathrm{G} 1$ & 4 & $\mathrm{Ka}$ & -471 & 146 & 487 & 70 & 493 \\
$2011 / 02 / 16$ & $\mathrm{Cm}$ & 5 & $\mathrm{~W}$ & $\mathrm{x}$ & $\mathrm{x}$ & $\mathrm{x}$ & $\mathrm{x}$ & 540 \\
$2011 / 03 / 14$ & $\mathrm{~F} 1$ & 6 & $\mathrm{Ka}$ & -397 & 10 & 379 & -117 & 397 \\
$2011 / 05 / 06$ & $\mathrm{C}$ & 7 & $\mathrm{Ka}$ & 526 & -138 & -537 & -83 & 543 \\
$2011 / 10 / 22$ & $\mathrm{D} 2$ & 8 & $\mathrm{Ka}$ & 243 & -141 & -112 & -256 & 280 \\
$2011 / 11 / 19$ & $\mathrm{G} 2$ & 9 & $\mathrm{Ka}$ & -455 & 119 & 446 & -140 & 470 \\
$2011 / 12 / 02$ & $\mathrm{H} 1$ & 10 & $\mathrm{Ka}$ & -233 & 156 & 212 & 287 & 280 \\
$2012 / 01 / 30$ & $\mathrm{~F} 2$ & 11 & $\mathrm{Ka}$ & -415 & 33 & 400 & -95 & 416 \\
$2012 / 02 / 19$ & $\mathrm{H} 2$ & 12 & $\mathrm{Ka}$ & -309 & 183 & 212 & 287 & 359 \\
\hline
\end{tabular}

Table 4.2: Chronological list of observing runs, as well, as offset of Iapetus from Saturn during those observing runs both in RA and Dec, and cross-elevation and elevation. Offsets not calculated for W-band observing. All units are arcseconds. The March 2010 observation was made as part of project AGBT10A_058, but all subsequent observations were part of project AGBT10C_043. 


\begin{tabular}{|l|c|c|c|c|c|c|c|c|c|c|}
\hline $\begin{array}{l}\text { Original/ } \\
\text { New Run }\end{array}$ & $1-\mathrm{A}$ & $2-\mathrm{B}$ & $3-\mathrm{D} 1$ & $4-\mathrm{G} 1$ & $6-\mathrm{F} 1$ & $7-\mathrm{C}$ & $8-\mathrm{D} 2$ & $9-\mathrm{G} 2$ & $11-\mathrm{F} 2$ & $12-\mathrm{G} 2$ \\
\hline 6 & 2 & 2 & 2 & 2 & - & - & - & - & - & - \\
7 & 2 & 1 & 1 & 1 & 2 & - & - & - & - & - \\
8 & 0 & 0 & 0 & 2 & 0 & 2 & - & - & - & - \\
9 & 0 & 0 & 2 & 2 & 0 & 3 & 0 & - & - & - \\
10 & 0 & 2 & 0 & 0 & 0 & 0 & 2 & 2 & - & - \\
11 & 0 & 2 & 0 & 0 & 0 & 2 & 2 & 2 & - & - \\
12 & 0 & 0 & 0 & 0 & 0 & 0 & 0 & 0 & 4 & 4 \\
\hline
\end{tabular}

Table 4.3: List of all observations of the null field performed. Each column corresponds to an original CCB observing run. Each row corresponds to a CCB observing run where observations were performed on the null field. For instance, this chart shows that during the 10th run, 2 null observations were made corresponding to the second run, which was position "B". The dashes represent observations which could not be made because the original observation had not yet occurred. Run 12 is an exception to this rule: since it was the last run, the null field used was on the precise opposite side of Saturn, which in the case of a symmetric beam would return the same sidelobe contamination, but inverted in sign.
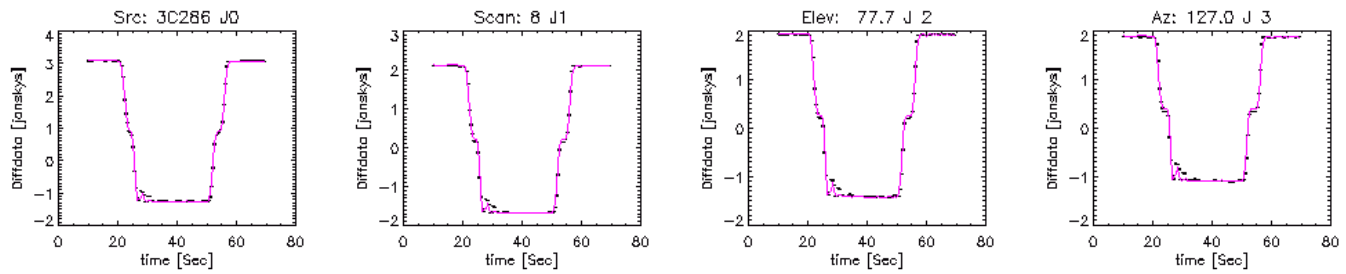

Figure 4.2: An example of a nod observation of the bright quasar 3C286 from run C. Longest wavelength observation is on the left. From 0-20 second, the first beam is on-source. From 20-25 seconds, the telescope is slewing to move the first beam off-source and the second beam on-source. From 25-50 seconds, the second beam is on-source and the first beam is off-source. From 50-55 seconds the beams are switched again and from 55 seconds onward, the first beam is again on-source with the second beam off-source. 


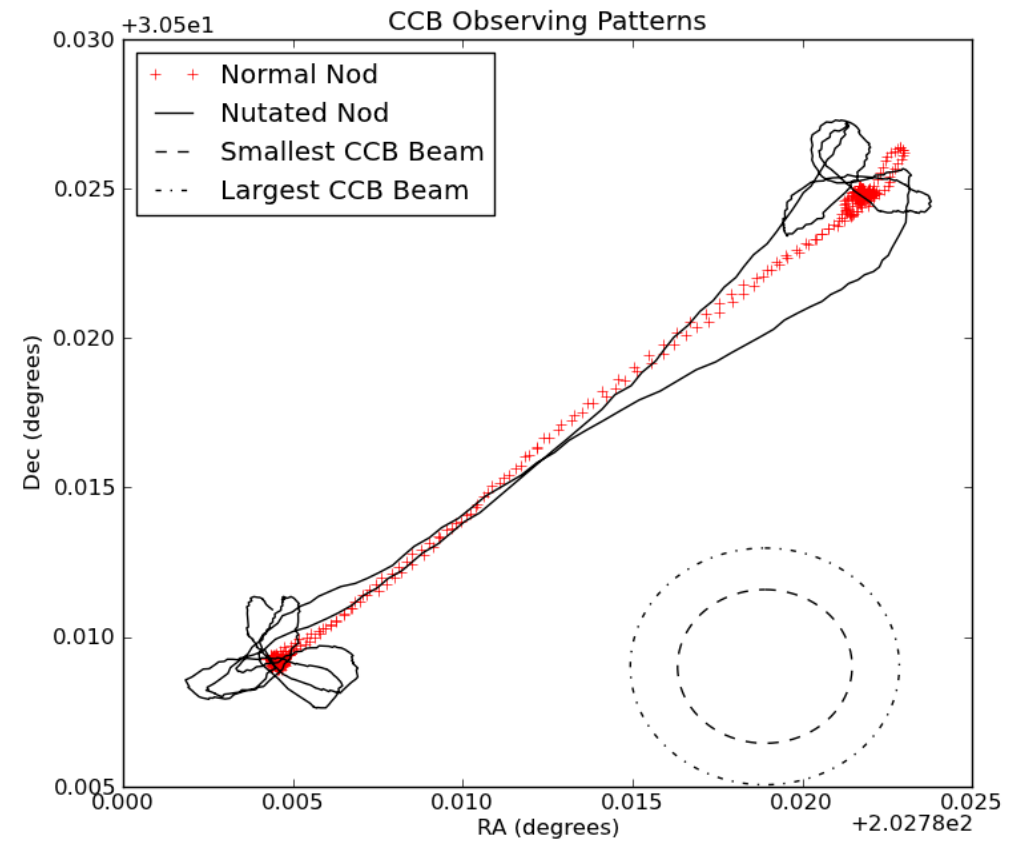

Figure 4.3: A comparison of the nutated and normal nod observing patterns for an observation of 3C286 during run $\mathrm{C}$, along with the beam sizes for the shortest and longest wavelengths observed by the CCB. 
distance and also easy to compare relative to a blackbody spectrum over multiple wavelengths.

One of the W-band observations (run $\mathrm{Cm}$ ) also used 3c286 as a calibrator, assuming a flux density of $746 \mathrm{mJy}$ (Steppe et al. 1995) at $90 \mathrm{GHz}$. However, the other MUSTANG observing run (run Gm) used less than ideal calibrators. One of the calibrators observed was asteroid 4 Vesta. However, as mentioned in Chapter 1, Vesta is a problematic calibrator at $3 \mathrm{~mm}$ as it has had a light curve detected at $3.2 \mathrm{~mm}$ (Müller and Barnes 2007), although the existence of this light-curve is still controversial. The problematic portion of Vesta's millimeter rotational light curve lies near $0^{\circ}$ longitude on its surface, and the most stable portion is centered near a longitude of $180^{\circ}$. Our own observations for this observing run fell near $115^{\circ}$ longitude, and thus they were unlikely affected by Vesta's rotational light-curve. Figure 4.4 shows our observations of Vesta during a different observing run along with the observations of Müller and Barnes (2007), with our observation near the edge of the light- curve drop scaled to $150 \mathrm{~K}$. Our data support the existence of scientifically interesting rotational light-curve for Vesta at $3 \mathrm{~mm}$, however, our data also demand caution when using Vesta as a calibrator.

On run Gm, we also serendipitously observed Titan, as it happened to be in the same 3 arcmin field-of-view as Iapetus. Could Titan act as a better calibrator? Titan has a nearly uniform disk-averaged brightness temperature of about 85K (Janssen et al. 2009) at $2 \mathrm{~cm}$. Unfortunately, observations at shorter wavelengths are complicated by the presence of Titan's atmosphere which has many spectral lines, as well as broad absorption. For example, early continuum measurements of Titan at 3.3 mm yielded a brightness temperature of 180K (Conklin et al. 1977) and a continuum brightness temperature measurement at $112 \mathrm{GHz}(2.6 \mathrm{~mm})$ of $69 \mathrm{~K}$ and a spectral 


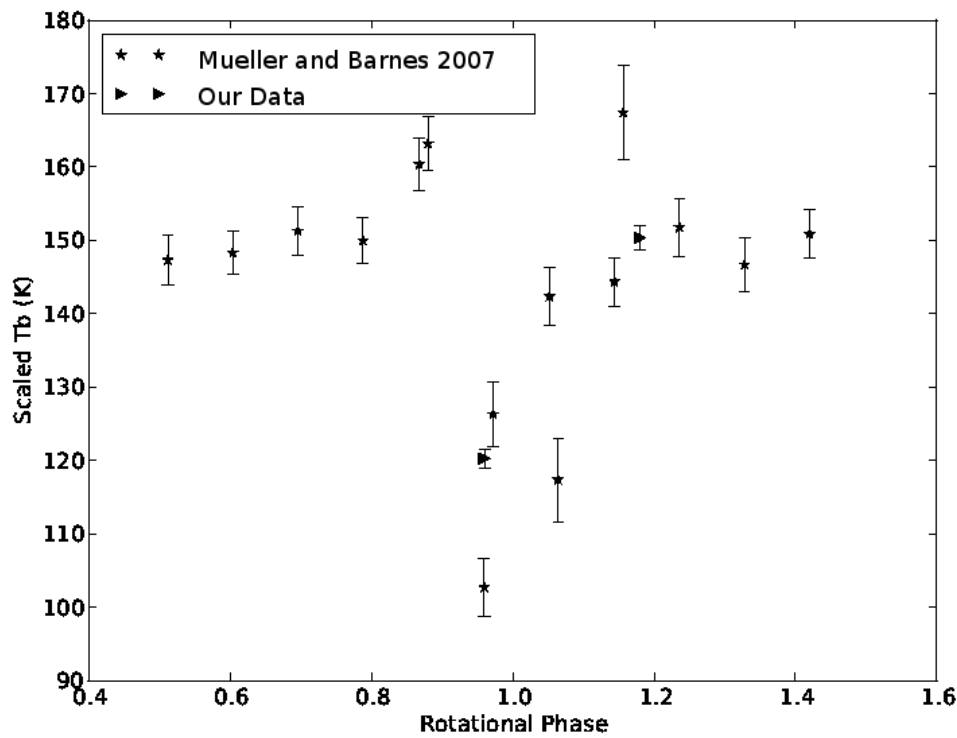

Figure 4.4: Plot of the rotational light curve of Vesta at $3.2 \mathrm{~mm}$ from Müller and Barnes (2007) along with our own observations from a MUSTANG observing run at $3.3 \mathrm{~mm}$. Our data are consistent with the existence of a rotational light-curve for Vesta at $\approx 3 \mathrm{~mm}$. 
line with brightness temperature 156K (Muhleman et al. 1984), which all produce substantially different results. Furthermore, there do not appear to be any more recent flux density measurements of Titan's continuum emission in the $3 \mathrm{~mm}$ range (see e.g. the review of Coustenis et al. (2010)), although additional spectral lines have been measured at $88.6 \mathrm{GHz}$ (Marten et al. 2002). Using an atmospheric model ${ }^{3}$, we calculate that the expected brightness temperature across the MUSTANG band, accounting for known and predicted spectral lines, is $82 \mathrm{~K}$.

To compare the calibrations, we used the observation of Titan to calibrate the brightness temperature of Vesta. Unfortunately, the two results were not in agreement, with the brightness temperature of Vesta derived from an $82 \mathrm{~K}$ Titan being 190K, whereas the brightness temperature derived from the measurements of Müller and Barnes (2007) is around 150K. Going the opposite direction, the observed $\mathrm{T}_{b}$ of Titan calibrated from Vesta is $62 \mathrm{~K}$.

So which calibrator is to be preferred? The answer is Vesta, as there are multiple reasons to think that the Titan observations might yield a lower brightness temperature than predicted, but none that would cause Vesta to have a higher brightness temperature than predicted. The atmosphere of Titan has already been described. The continuum brightness temperature of Titan might be lower than that predicted from the model as no comparable observations exist to constrain the $80-100 \mathrm{GHz}$ brightness temperature, and the main effect of the atmosphere on continuum observations is to lower the observed brightness temperature as higher frequency observation probe higher in the atmosphere. Another possible reason is an observational one. Titan was near the edge of the field of view of a map centered on Iapetus. The MUSTANG reduction algorithms may smooth out (i.e. reduce the brightness of) any source outside the inner 30 or so arcsec of the field, as they use the outer data of the

\footnotetext{
${ }^{3}$ This particular model is a part of the CASA 3.4 software package.
} 
field to model the sky background (i.e. much like a nod is used in the CCB). Vesta's only problem is its light curve, which involves reduced brightness temperature, rather than an increased one as a Titan calibration would require. Furthermore, Vesta was observed at "boring" geometry with regards to the observed light curve. Therefore, since the Vesta observations are of higher quality, with Vesta in the center of the field, and since Titan has substantial observational and model uncertainty, Vesta will be used for calibration of run Gm.

\subsubsection{Saturn as a Sanity Check}

Saturn was observed repeatedly on several nights of CCB observations to ensure that all bands of the instrument were functioning properly. Saturn is mostly a blackbody at microwave frequencies,(Weiland et al. 2011) so any frequency-dependent calibration error should appear in the Saturn data. For these calculations, Saturn was assumed to be a blackbody of $140 \mathrm{~K}$ at all four frequencies. The radius of Saturn was assumed to be $57240 \mathrm{~km}$, which is a circular source with equivalent area to the reference solid angle of $5.096 \times 10^{-9}$ sr at $9.5 \mathrm{AU}$ used by Weiland et al. (2011). The contribution of the rings was neglected, although at the ring opening angles during this project $\left(10-15^{\circ}\right)$, they may cause a slight, uniform absorption at Ka-band of no more than 7-8K (Weiland et al. 2011). These values from the literature were then converted to flux density using the Rayleigh-Jeans limit equations from 1.3. Figure 4.5 shows observations of Saturn during the last four of Ka-band observing runs. Saturn is clearly a blackbody, except in the case of run 10 (H1), where the instrument suffered a malfunction. In the case of run 9 (G2), Saturn's predicted flux density was carefully calculated based on the brightness temperature of Weiland et al. (2011) and the change in beam coupling with frequency. The resulting residuals were less than $3 \%$ in all bands, implying 

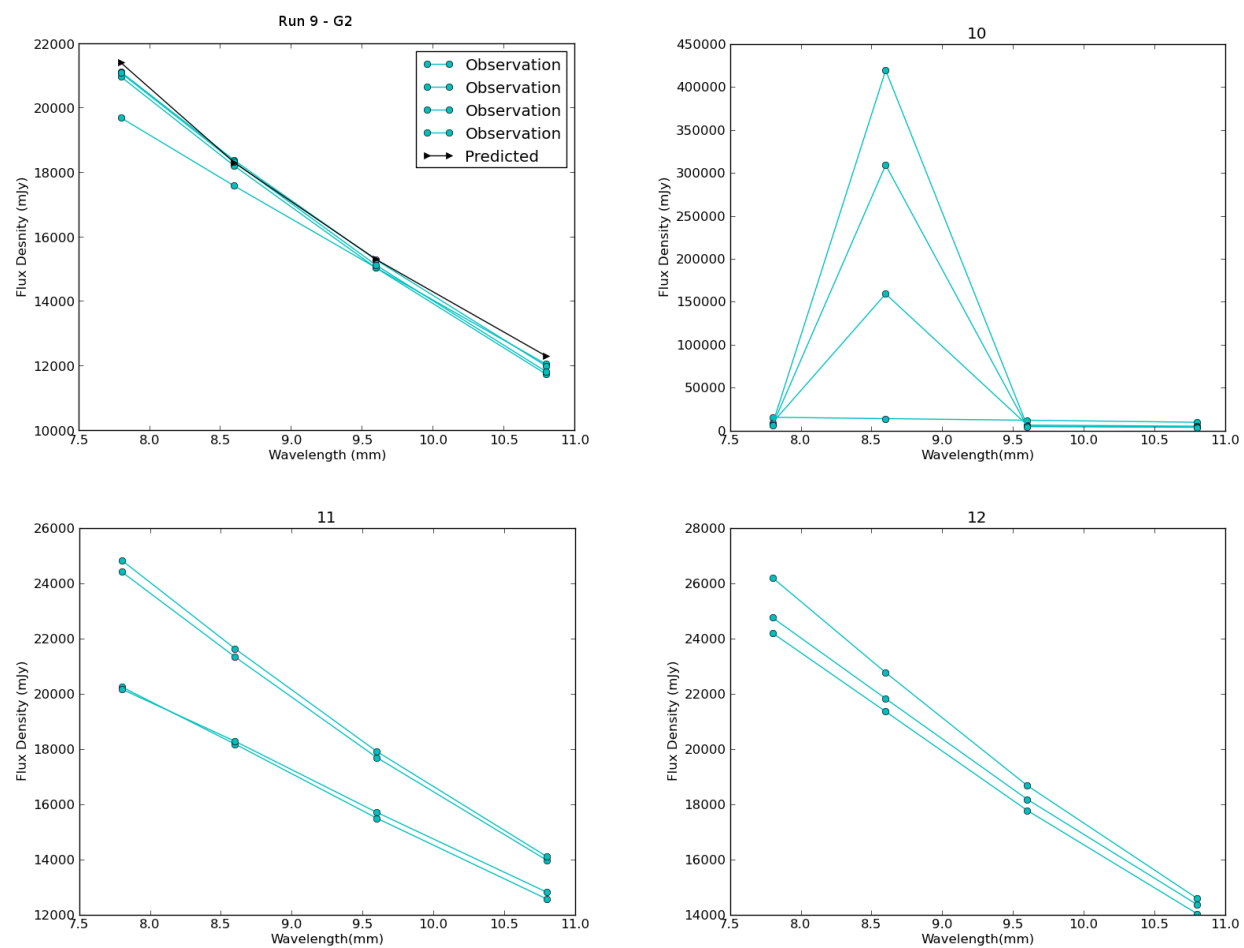

Figure 4.5: Sanity checks on Saturn. In all of the runs except run 10 (where there was an instrument malfunction), Saturn came back as a blackbody, ruling out instrumental artifacts. In run 9, the predicted flux density of Saturn was also calculated based on the data of Weiland et al. (2011) and accounting for beam coupling. The residuals were less than $3 \%$ in all bands. There is a possible opacity change or pointing error during run 11. Runs 9 and 11 correspond to longitudinal locations G2 and F2, respectively.

that both the blackbody fidelity and flux density calibration from 3C286 are accurate to that level. The Saturn observations provide strong evidence against any unusual spectral shapes being the result of instrumental error, and also corroborate the flux density calibration from 3C286. 


\subsection{Sidelobe Mitigation Technique}

\subsubsection{Definitions and overview}

This technique was developed primarily in order to measure the flux density of various moons in the solar system. As such, the descriptions in this section will reference a "planet" and "moon" generically, where the "planet" is a source which is many orders of magnitude brighter than the "moon" and may possibly be resolved. The main concern in obtaining flux density of the moon is the contamination of the moon's signal with the signal of the planet due to the planet being located in a sidelobe of the telescope's main beam. While sidelobes are several orders of magnitude weaker than the main beam, they can still be relevant for a moon which is also similar orders of magnitude fainter than the planet.

One way to look for significant sidelobe contamination is to conduct observations at multiple epochs. Since the moon moves around the planet, we can conduct observations when the moon is existent at a location and when the moon is non-existent at a location and compare the two in order to determine if sidelobes are playing a role. Figure 4.6 illustrates the technique and the outline of the algorithm used is given below:

1. Obtain Ra and Dec offset of moon relative to planet at the time of original observation from HORIZONS ephemeris system. ${ }^{4}$

2. Using the time of observation, location of the GBT, Ra/Dec offset of the moon, and J2000 ephemeris of the moon during the first epoch of observations calculate the elevation and cross-elevation offset of moon relative to the planet at the midpoint of the first epoch of observations.

\footnotetext{
${ }^{4}$ http://ssd.jpl.nasa.gov/horizons.cgi
} 

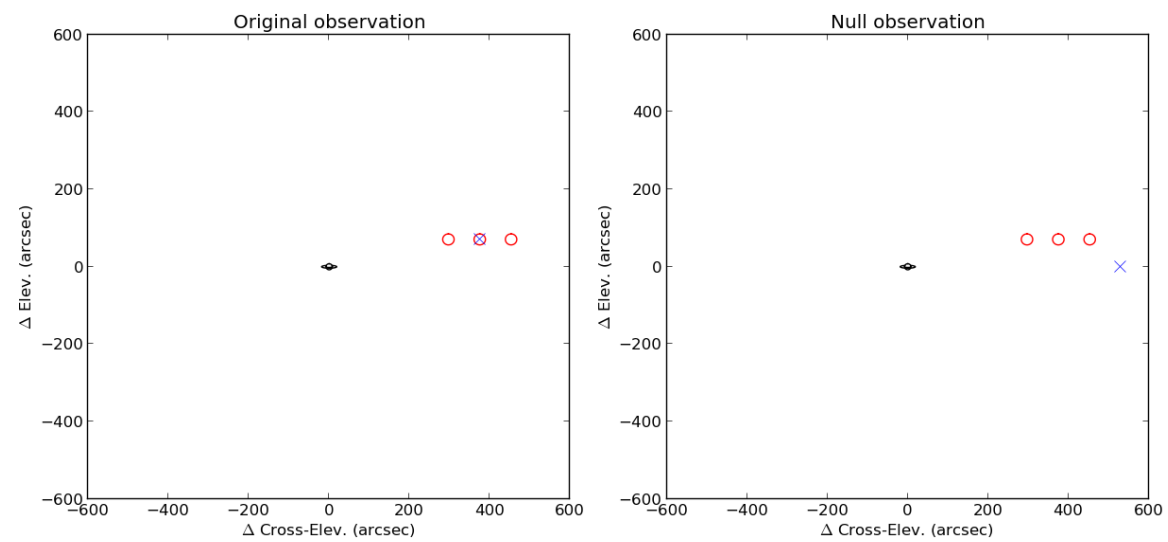

Figure 4.6: Illustrative plot of the sidelobe technique. In the figures, the red circles represent the positions of the two telescope beams during a beam switching nod, and the $\mathrm{x}$ represents the location of the target moon. The figure on the left shows a normal CCB nod on a moon. The figure on the right shows the corresponding check for sidelobe contamination ("null observation") where the relative el and crossel observing locations are the same, but the moon is no longer located in any of the beam locations, either due to orbital motion or a change in the parallactic angle.

3. Obtain Ra and Dec ephemeris of the planet at the time of a subsequent observing epoch from HORIZONS.

4. Create a new ephemeris file with an RA and Dec corresponding to a constant elevation and cross-elevation offset from the planet at the time of the second epoch of observation.

5. Nod on previous location at same relative elevation/cross-elevation to the planet as previous scan.

In addition to the fact that the moon is no longer at the observed location, the parallactic angle will likely be different, which is especially relevant for objects which do not change positions and will be discussed in detail later. 


\subsubsection{Specific Example: Saturn's moon Iapetus}

This technique was developed as a part of a GBT observing project (AGBT10C-043) to measure Iapetus' rotational light curve. The checks for sidelobe contamination were conducted and were also compared to "theoretical" results. Figure 4.7 shows the location of the various nods on Iapetus compared to a "theoretical" beam map at CCB frequencies. The reason "theoretical" is in quotes is because the model used is based on both observational and theoretical results. The primary data used to construct the map comes from a 2-degree, high dynamic range map obtained at 11.7 GHz in the early morning of September 11, 2009(Hunter et al. 2011). This map was then converted, using holographic techniques, into a model of the telescope surface (Hunter et al. 2011). This holographic data was then Fourier transformed into beam maps at many different frequencies within the CCB range of 26-40 GHz. These maps were convolved with a Saturn-sized (15.8 arcsecond diameter) disk. Beam maps were produced for each band by stacking 30 single-frequency beam maps at evenly spaced frequencies across each of the four individual CCB bands (26-29.5 GHz, 29.5-33 GHz, 33-36.5 GHz, 36.5-40 GHz). ${ }^{5}$ The resulting model for each CCB band predicts the expected contribution of contamination from Saturn to measured flux out to a distance of 15-20 arcminutes from Saturn, which is more than adequate for our observations, which extend no farther than 9 arcmin from Saturn. This model should be representative of a good, thermally stable nighttime surface.

Saturn has a flux density of order 10-20 Jy in the CCB bands, whereas Iapetus has a flux density of only 1-4mJy, leading to a contrast between the two of order $10^{4}$. The only location where the contamination from Saturn is expected to be at or

\footnotetext{
${ }^{5}$ Experiments were conducted as to the number of single-frequency maps necessary to fully account for the smoothing of sharp beam features created by the large bandwidth of the CCB, and it was determined that there was little variation in the combined maps above ten single-frequency maps combined.
} 

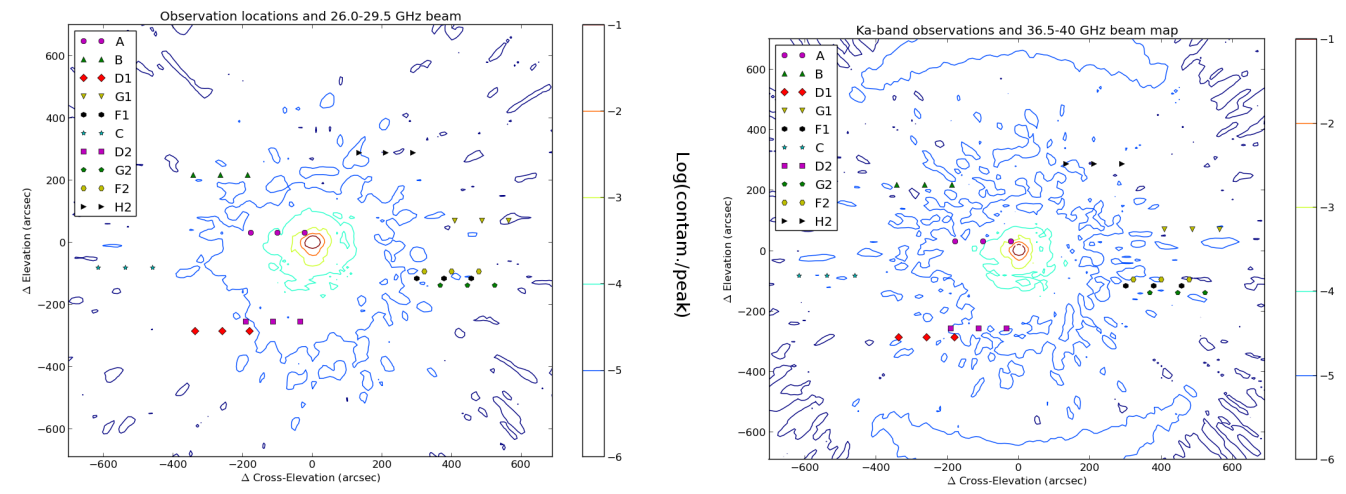

Figure 4.7: Maps of the GBT beam at the lowest (left) and highest (right) frequency bands observable with the CCB convolved with a 16 arcsecond uniform disk.

greater than this value is at location "A." Figure 4.8 shows several null observations. The only location with qualitatively obvious sidelobes detected was location "A."

The predicted contribution of sidelobes is the difference in the difference of the right offset beam and on-source and the difference of left offset beam and on-source. Figure 4.9 shows some example predicted sidelobes. Theory predicts that sidelobe contamination at point A will be of order 30-40 mJy. The actual measured sidelobe contamination is of the same order of magnitude (10-50 mJy), which indicates that the theory results are plausible and useful. At other all other locations, the greatest theoretical contribution from sidelobes is of order a few tenths of a mJy.

The parallactic angle is not constant over the course of an observing run. Could the change in parallactic angle result in encounters with more sidelobe contamination? Figure 4.10 shows the evolution of of the sidelobe contamination over time at two different ra/dec offsets from Saturn. The change in sidelobe contamination is substantial close to Saturn, but the changes are minimal at more distant (and more scientifically interesting) locations. Tables 4.4, 4.5, and 4.6 show the maximum deviation, the mean deviation, and the extra noise, respectively contributed by sidelobes 

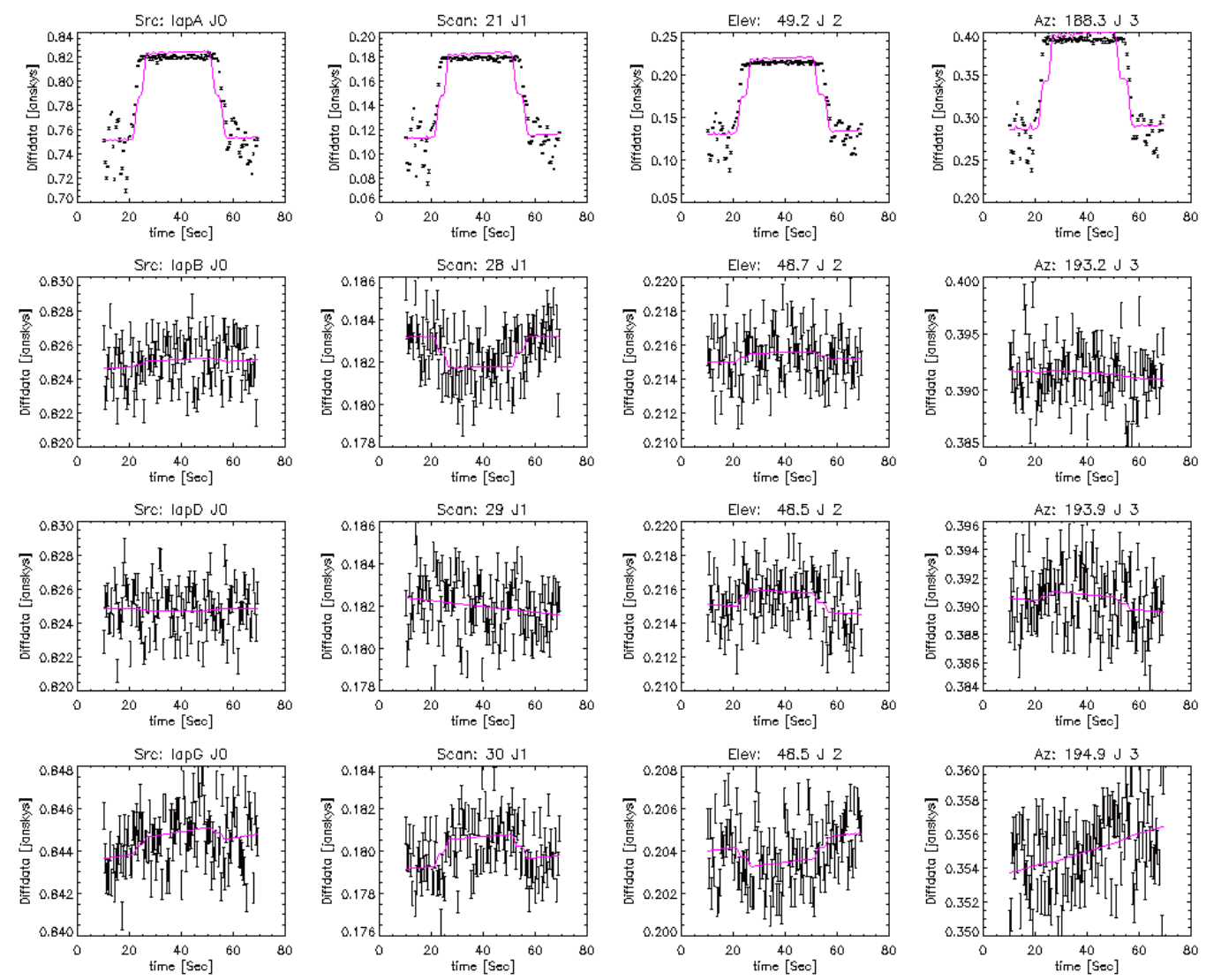

Figure 4.8: Example sidelobe checking observations. Columns show the four different frequency bands of the CCB, with the left being lowest frequency/longest wavelength. Each row shows a different sidelobe-checking observation. The magenta lines show the best fit to the data. The only clear detection of sidelobe contamination is in the first case, when Iapetus was closest to Saturn on the sky. 

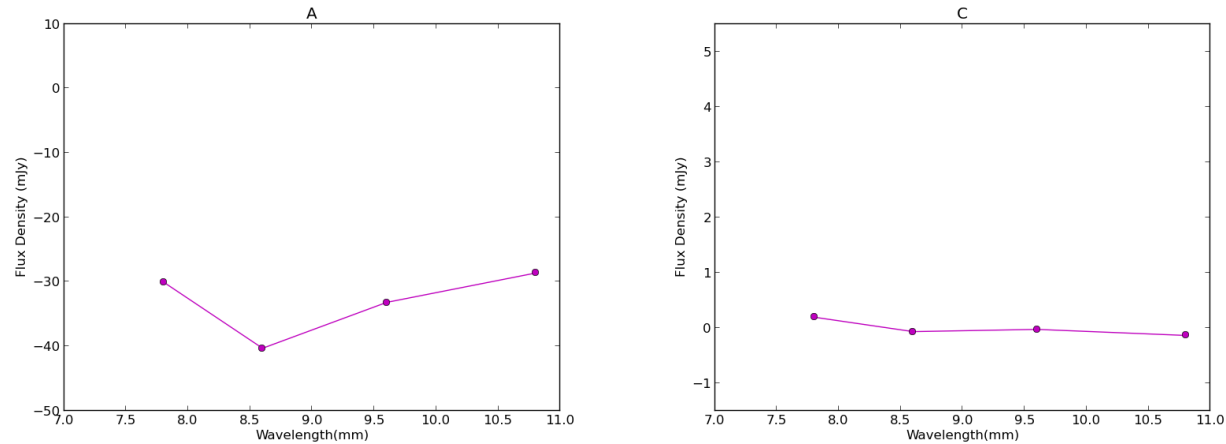

Figure 4.9: Examples of expected sidelobe contamination at the closest $(\mathrm{A})$ and farthest (C) points from Saturn observed.

\begin{tabular}{|l|r|r|r|r|}
\hline Run let. & $10.8 \mathrm{~mm}$ & $9.6 \mathrm{~mm}$ & $8.6 \mathrm{~mm}$ & $7.8 \mathrm{~mm}$ \\
\hline A & 464.9979 & 408.3088 & 509.7012 & 436.6017 \\
G1 & 0.1114 & 0.1262 & 0.1848 & 0.3701 \\
F1 & 0.1487 & 0.2418 & 0.1769 & 0.2108 \\
C & 0.0905 & 0.1320 & 0.3735 & 0.3457 \\
D2 & 0.3429 & 0.4696 & 0.3656 & 0.4147 \\
G2 & 0.1095 & 0.1245 & 0.1464 & 0.2501 \\
F2 & 0.1244 & 0.2843 & 0.2387 & 0.3112 \\
\hline
\end{tabular}

Table 4.4: Maximum predicted contribution of Saturn flux to Iapetus due to sidelobes in mJy during the course of an observing run.

according to theory. The mean and noise contributions are at most about $0.1 \mathrm{mJy}$ per beam, except in the case of location "A", and thus no significant sidelobe contamination is expected from the known beam map. It is possible that actual sidelobe contamination may be worse than predicted, as several observations occurred during daylight hours where asymmetric solar heating can deform the dish shape away from the stable nighttime shape used in the beammap.

The final test for sidelobe contamination in the Iapetus data is to observationally look for statistically significant sidelobes at locations when Iapetus is not present, as described above. For statistical significance, a z-test was used. The main usage of the z-test is to determine if two normally distributed data sets of similar $\sigma$ are 

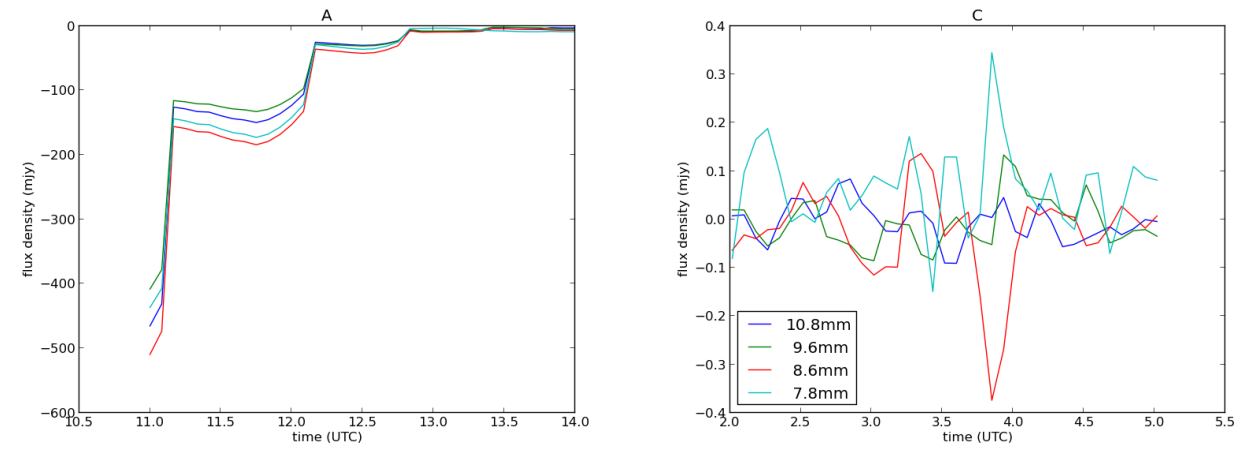

Figure 4.10: Examples of expected sidelobe contamination at the closest (A) and farthest (C) points from Saturn observed and their evolution over the course of their respective observing runs. $\mathrm{C}$ is a prototypical example in that the variation is centered around 0 and less than $10 \%$ of the expected flux density of Iapetus.

\begin{tabular}{|l|r|r|r|r|}
\hline Run let. & $10.8 \mathrm{~mm}$ & $9.6 \mathrm{~mm}$ & $8.6 \mathrm{~mm}$ & $7.8 \mathrm{~mm}$ \\
\hline A & -76.1347 & -68.7826 & -91.4973 & -82.2498 \\
G1 & 0.0011 & -0.0071 & -0.0357 & -0.0583 \\
F1 & -0.0249 & -0.0116 & -0.0247 & -0.0265 \\
C & -0.0064 & -0.0085 & -0.0275 & 0.0651 \\
D2 & 0.1402 & 0.2136 & -0.0451 & -0.1162 \\
G2 & -0.0059 & -0.0181 & -0.0152 & -0.0173 \\
F2 & 0.0132 & -0.0081 & -0.0245 & 0.0074 \\
\hline
\end{tabular}

Table 4.5: Mean predicted contribution of Saturn flux to Iapetus due to sidelobes in mJy during the course of an observing run.

\begin{tabular}{|l|r|r|r|r|}
\hline Run let. & $10.8 \mathrm{~mm}$ & $9.6 \mathrm{~mm}$ & $8.6 \mathrm{~mm}$ & $7.8 \mathrm{~mm}$ \\
\hline A & 105.4480 & 92.7994 & 118.4126 & 104.2482 \\
G1 & 0.0479 & 0.0646 & 0.0617 & 0.1234 \\
F1 & 0.0545 & 0.1188 & 0.0698 & 0.1220 \\
C & 0.0384 & 0.0494 & 0.0941 & 0.0871 \\
D2 & 0.1077 & 0.1864 & 0.1092 & 0.1429 \\
G2 & 0.0549 & 0.0609 & 0.0710 & 0.1027 \\
F2 & 0.0487 & 0.1158 & 0.0916 & 0.1336 \\
\hline
\end{tabular}

Table 4.6: Noise contribution from Saturn flux (standard deviation) at Iapetus due to sidelobes in mJy during the course of an observing run. 
significantly different from each other. The most useful and important feature of the z-test, however, is the fact that it can be used on very small data sets (it reduces to the normal distribution for a single data point). The formula for the z-test is $z=\frac{\left|\overline{x_{0}}-\overline{x_{1}}\right|}{\sigma} \sqrt{n}$, where $\overline{x_{0}}$ is the mean of the first data set (in this case assumed to be zero, as gaussian noise should average to zero), $\overline{x_{1}}$ is the mean of the sidelobe contamination, $\sigma$ is the standard deviation of the noise measurements, and $n$ is the number of sidelobe observations. The resulting $\mathrm{p}$-value is then just $p=1-\operatorname{erf}\left(\frac{z}{\sqrt{2}}\right)$, where $\operatorname{erf}()$ is the error function. On each night, only 1-3 sidelobe contamination checks on each of the previously observed locations were performed. The precise statistical result sought was to determine if the sidelobe contamination data were significantly different from the noise on the night they were observed. The noise was calculated by subtracting mean value observed fluxes of Iapetus on each night in each channel from each individual Iapetus observation. This residual distribution was used to calculate the noise on each night. Tables 4.7, 4.8, 4.9, 4.10 show the statistical significance of sidelobe contamination. Essentially, the only statistically significant contamination was found at location A, although location D2 also showed some significant sidelobe contamination.

The detected contamination at D2 is slightly troubling, in that it is several times larger than predicted by theory. On the one hand, the D2 science measurements are of little scientific value to begin with due to poor weather on the day of the original observation. However, the fact that the detected sidelobe contamination is stronger than predicted by theory at one location implies that there might be stronger sidelobes elsewhere. This concern is alleviated by the sidelobe contamination checks performed at the other Iapetus observation locations which showed no statistically significant sidelobe contamination. Therefore, except for locations D2 and A, sidelobe 
contamination can be ruled out as a substantial contribution to the observations.

\subsubsection{General Sidelobe mitigation technique}

The sidelobe mitigation technique is not solely applicable to solar system objects, which conveniently move to new locations at regular intervals. For any astronomical object, the fact that the GBT is set up on an altazimuth mount leads to the possibility of using changing parallactic angle to achieve a similar outcome. For any pair of sources in the sky with a fixed offset in RA and Dec., the same sources will change their offset in altitude and azimuth as they trace their path through the sky. The maximum change in parallactic angle is a function of the observer's latitude and the declination of the source. This produces a limit on what sources for which this technique is applicable. For determining the limiting declination at various different

frequencies, we assume that the minimum required rotation is the angle required to move one beam at a distance of 2 beams from the brighter source. This angle is $29.0^{\circ}$, which is then divided by two in order to account for the fact the parallactic angle is reversed as an object crosses the meridian. Fig. 4.11 shows parallactic angles for sources at several different limiting elevations at the location of the GBT versus declination. This technique should therefore be useful on almost any source realistically observable by the GBT. This technique should be applicable to all but the lowest declination of sources, although it would be somewhat more constrained in practice by length of observing run than absolute parallactic angle limit.

\subsubsection{Application to mapping}

This technique is applicable to mapping as well. Most astronomical maps are produced in celestial coordinates. However, if two different maps are produced at different 
parallactic angles and in horizontal coordinates instead, one map can be subtracted from the other to remove any effects of the antenna beam pattern created by the brighter of the two sources. 


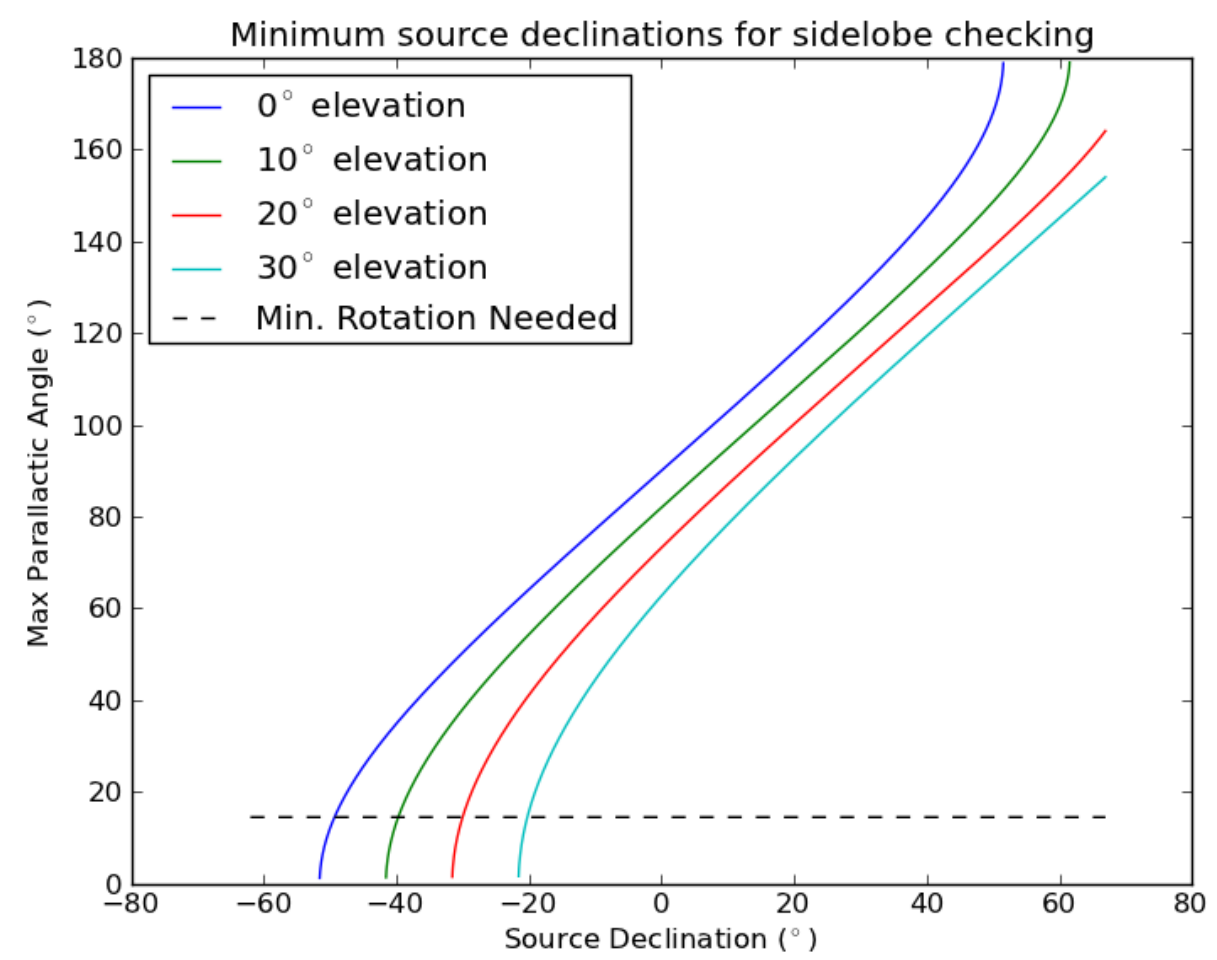

Figure 4.11: Plot of maximum parallactic angle versus declination for several different minimum elevation observing limits at the location of the GBT. Almost any source should rotate enough in azimuth and elevation for this technique to be applicable. Note the limits at low declination are caused by sources not rising above the horizon and the limit at high declination is caused by an object becoming circumpolar at the GBT longitude. Note that while an object of declination exactly equal to the latitude of the telescope $\left(38^{\circ} \mathrm{N}\right)$ will exist at all azimuths as it transits the zenith, this is a special case as all other declinations do not allow for parallactic angles other than $0^{\circ}$ or $180^{\circ}$ at or near transit. 


\begin{tabular}{|l|c|c|c|c|c|c|c|c|}
\hline $\begin{array}{l}\text { Original/ } \\
\text { New Run }\end{array}$ & 1-A & $2-\mathrm{B}$ & $3-\mathrm{D} 1$ & $4-\mathrm{G} 1$ & $6-\mathrm{F} 1$ & $7-\mathrm{C}$ & $8-\mathrm{D} 2$ & $9-\mathrm{G} 2$ \\
\hline 6 & 0.0 & 0.97 & 0.62 & 0.63 & - & - & - & - \\
7 & 0.0 & 0.50 & 0.75 & 0.10 & 0.14 & - & - & - \\
8 & - & - & - & 0.93 & - & 0.98 & - & - \\
9 & - & - & 0.26 & 0.90 & - & 0.75 & - & - \\
11 & - & 0.55 & - & - & - & 0.28 & 0.06 & 0.44 \\
\hline
\end{tabular}

Table 4.7: P-value of sidelobe contamination determined via z-test at $10.8 \mathrm{~mm}$.

\begin{tabular}{|l|c|c|c|c|c|c|c|c|}
\hline $\begin{array}{l}\text { Original/ } \\
\text { New Run }\end{array}$ & 1-A & 2-B & $3-\mathrm{D} 1$ & $4-\mathrm{G} 1$ & $6-\mathrm{F} 1$ & $7-\mathrm{C}$ & $8-\mathrm{D} 2$ & $9-\mathrm{G} 2$ \\
\hline 6 & 0.0 & 0.47 & 0.38 & 0.31 & - & - & - & - \\
7 & 0.0 & 0.03 & 0.95 & 0.04 & 0.67 & - & - & - \\
8 & - & - & - & 0.55 & - & 0.99 & - & - \\
9 & - & - & 0.22 & 0.16 & - & 0.16 & - & - \\
11 & - & 0.14 & - & - & - & 0.56 & $1 \mathrm{e}-3$ & 0.49 \\
\hline
\end{tabular}

Table 4.8: P-value of sidelobe contamination determined via z-test $9.6 \mathrm{~mm}$.

\begin{tabular}{|l|c|c|c|c|c|c|c|c|}
\hline $\begin{array}{l}\text { Original/ } \\
\text { New Run }\end{array}$ & 1-A & $2-\mathrm{B}$ & $3-\mathrm{D} 1$ & $4-\mathrm{G} 1$ & $6-\mathrm{F} 1$ & $7-\mathrm{C}$ & $8-\mathrm{D} 2$ & $9-\mathrm{G} 2$ \\
\hline 6 & 0.0 & 0.18 & 0.22 & 0.40 & - & - & - & - \\
7 & 0.0 & 0.39 & 0.10 & 0.27 & $6 \mathrm{e}-3$ & - & - & - \\
8 & - & - & - & 0.44 & - & 0.47 & - & - \\
9 & - & - & 0.50 & 0.06 & - & 0.86 & - & - \\
11 & - & 0.34 & - & - & - & 0.64 & $6 \mathrm{e}-3$ & 0.27 \\
\hline
\end{tabular}

Table 4.9: P-value of sidelobe contamination determined via z-test at $8.6 \mathrm{~mm}$.

\begin{tabular}{|l|c|c|c|c|c|c|c|c|}
\hline $\begin{array}{l}\text { Original/ } \\
\text { New Run }\end{array}$ & 1-A & $2-\mathrm{B}$ & $3-\mathrm{D} 1$ & $4-\mathrm{G} 1$ & $6-\mathrm{F} 1$ & $7-\mathrm{C}$ & $8-\mathrm{D} 2$ & $9-\mathrm{G} 2$ \\
\hline 6 & 0.0 & 0.30 & 0.40 & 0.40 & - & - & - & - \\
7 & 0.0 & 0.89 & 0.48 & 0.76 & 0.51 & - & - & - \\
8 & - & - & - & 0.82 & - & 0.97 & - & - \\
9 & - & - & 0.48 & 0.76 & - & 0.67 & - & - \\
11 & - & 0.29 & - & - & - & 0.91 & 0.03 & 0.95 \\
\hline
\end{tabular}

Table 4.10: P-value of sidelobe contamination determined via z-test $7.8 \mathrm{~mm}$. 


\begin{tabular}{|c|c|c|c|c|c|c|}
\hline Run Let. & $10.8 \mathrm{~mm} \sigma$ & $9.6 \mathrm{~mm} \sigma$ & $8.6 \mathrm{~mm} \sigma$ & $7.8 \mathrm{~mm} \sigma$ & Run No. & Qual. \\
\hline $\mathrm{C}$ & 0.169435 & 0.251282 & 0.17184 & 0.386994 & 7 & $\mathrm{G}$ \\
$\mathrm{B}$ & 0.388449 & 0.309896 & 0.314386 & 0.74521 & 2 & $\mathrm{M}$ \\
$\mathrm{G} 2$ & 0.643781 & 0.203079 & 0.364742 & 0.548368 & 9 & $\mathrm{G}$ \\
$\mathrm{D} 1$ & 0.268068 & 0.206257 & 0.505029 & 0.316111 & 3 & $\mathrm{G}$ \\
$\mathrm{F} 1$ & 0.176948 & 1.05724 & 0.556116 & 0.68693 & 6 & $\mathrm{M}$ \\
$\mathrm{F} 2$ & 0.375467 & 0.300658 & 0.779319 & 1.08666 & 11 & $\mathrm{M}$ \\
$\mathrm{G} 1$ & 0.425032 & 0.646723 & 1.00922 & 0.850972 & 4 & $\mathrm{M}$ \\
$\mathrm{D} 2$ & 0.450878 & 0.657438 & 1.23496 & 1.17662 & 8 & $\mathrm{~F}$ \\
$\mathrm{~A}$ & 100 & 100 & 100 & 100 & 1 & $\mathrm{~F}$ \\
$\mathrm{H} 2$ & 100 & 100 & 100 & 100 & 12 & $\mathrm{~F}$ \\
\hline
\end{tabular}

Table 4.11: Noise for each observing run, sorted by noise in the $8.6 \mathrm{~mm}$ band calculated on the basis of the the procedure outlined in section 4.4 of Mason et al. (2009). All noises are in mJy. The quality of each run is either good $(G)$, marginal (M), or failed (F). Good=good signal to noise detection. Marginal=clear detection of Iapetus, but with poor constraint on flux density. Fail=dubious detection or non-detection. The run at location B was marginal since it had only a small number of observations rather than a high noise level.

\section{$4.41 \mathrm{~cm}$ Observational Results}

Our Ka-band/CCB observations generally fall into three categories: good, marginal, and failed. The good observations are shown in Figure 4.13, the marginal observations are shown in Figures 4.14 and 4.15, and the failed observations are shown in Figures 4.17 and 4.18. The primary criterion dividing these categories is weather and thus the measurement uncertainty on each night. Table 4.11 shows the runs sorted by noise level in the $8.6 \mathrm{~mm}$ band. Figure 4.12 shows the three longitudes with good data plotted on a map of Iapetus.

The average flux density and brightness temperature data from each night are given in Table 4.4. Some plot characteristics are shared in common with all of the runs. All are shown with all of the individual observations (cyan), the average and error derived from those observations (black) and the re-observations of the null field (magenta) taken during later observation runs at the same elevation and 


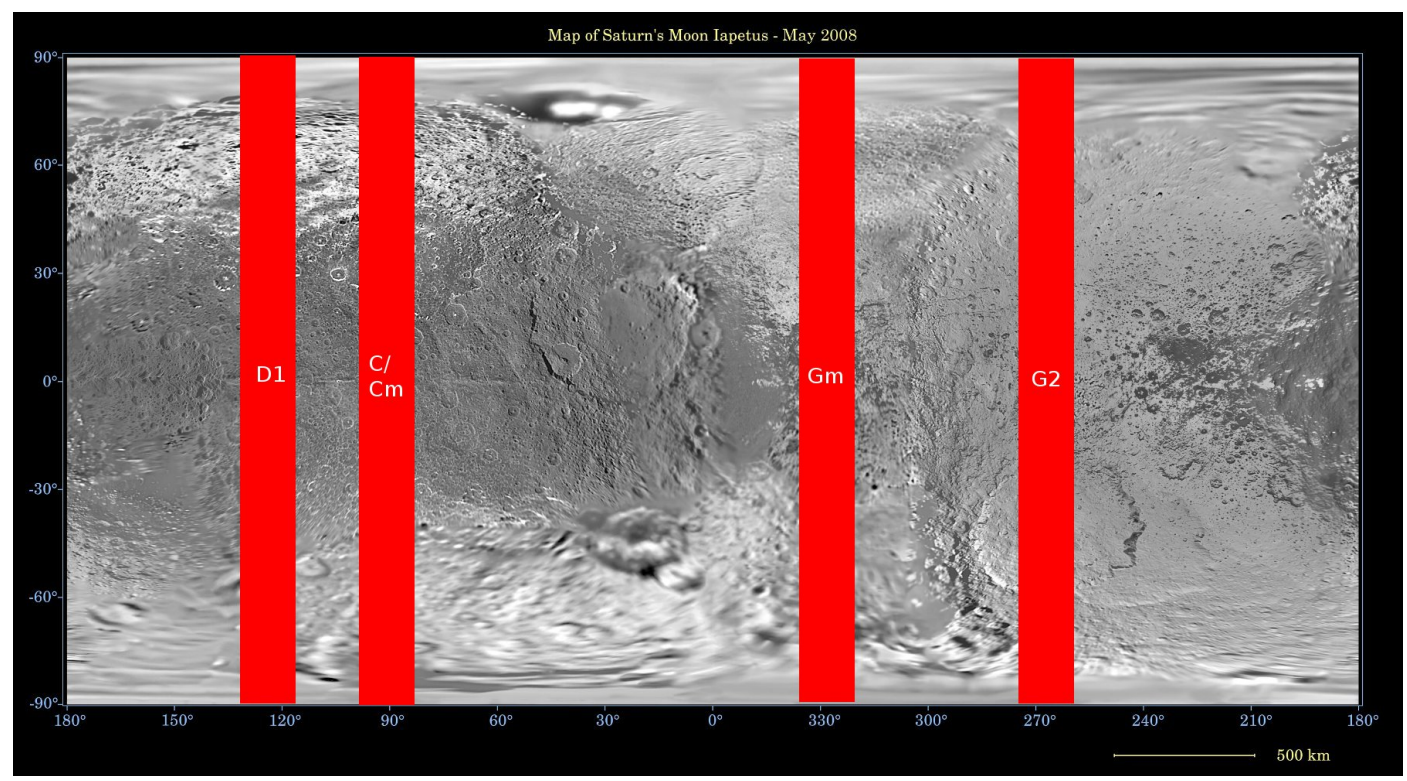

Figure 4.12: Plot of locations of all of the good observations of Iapetus with the CCB as well as with the MUSTANG bolometer array.

cross-elevation offsets from Saturn.

Some observations indicate a "negative" flux density. Obviously, a negative flux density is unphysical, but these results are still "real" to an extent. As described earlier, a null observation takes the difference in difference between when the first beam is on source and when the second beam is on source. In some cases, the flux density in the first beam when the first beam is off-source may be greater than than the flux density of the source itself, such as when a sidelobe hits Saturn. A nondetection can also show up as a negative flux density, as the fitting routines are effectively just fitting the noise in a difference, and the result can be either slightly positive or slightly negative (the run 12 observation seen in Figure 4.17 is a good example).

The best three observations are self-consistent. Observations on Iapetus' dark leading side seem to be showing roughly constant brightness temperature across the 

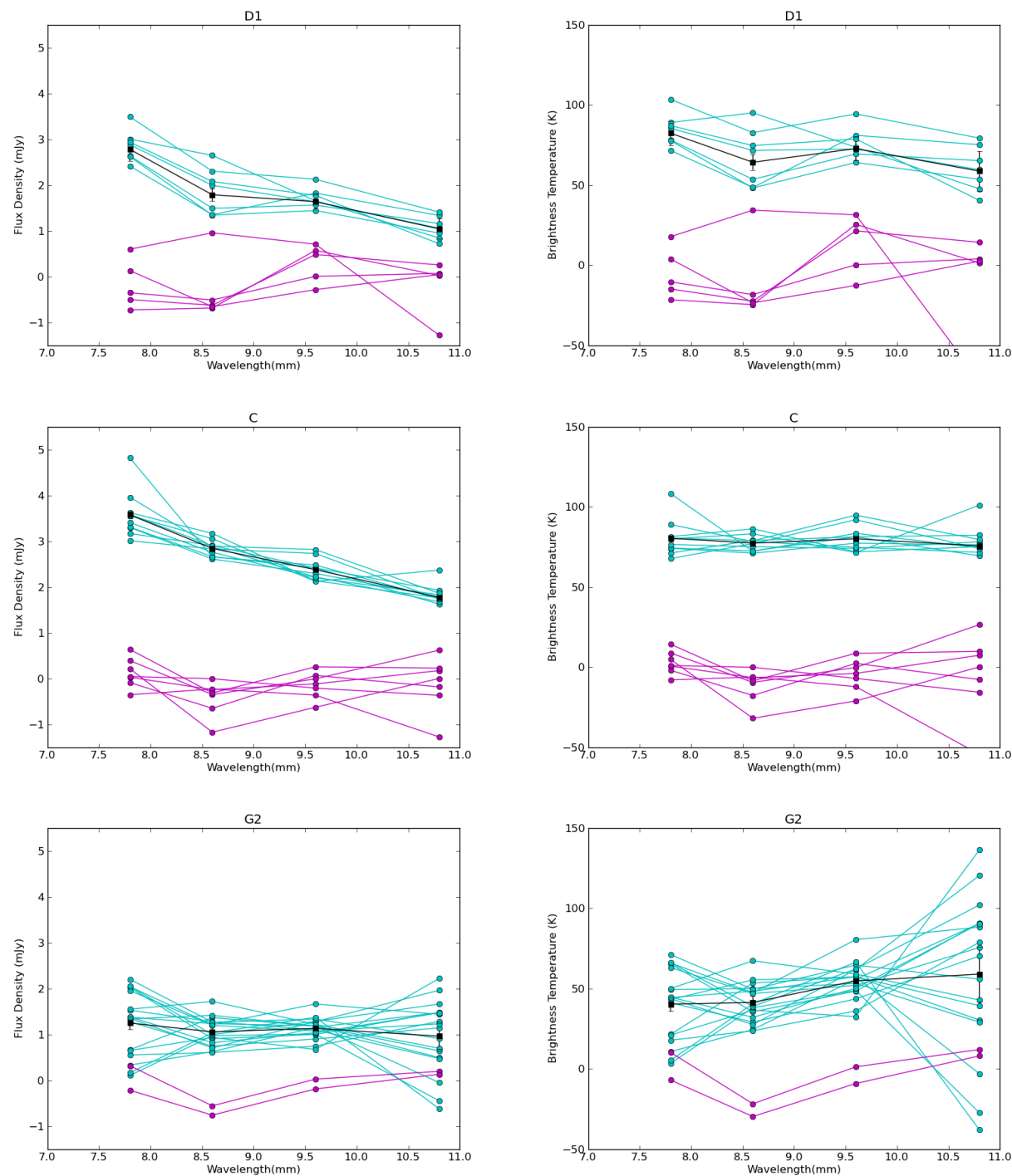

Figure 4.13: The left column shown in this plot and several similar plots below shows flux densities, which will vary with distance between Earth and Iapetus. The right column shows brightness temperatures, which are independent of distance and also show any variation relative to a blackbody. Good observing runs. Magenta: Null observations. Cyan: Individual observations. Black: Average measurements with error bars. In all of these cases the scatter in the observed data is modest and the null observations are not inconsistent with zero. Run $\mathrm{C}$ is consistent with the same spectral energy distribution in run D1, which is to be expected given that they are both dominated by the dark material. 
$1 \mathrm{~cm}$ band. Observations on Iapetus' trailing hemisphere show a roughly constant flux density across the $1 \mathrm{~cm}$ band, which corresponds to a steep drop in brightness temperature at shorter wavelengths. The $\mathrm{C}$ and D1 observations are not statistically distinguishable using a two-distribution $\chi^{2}$ test, with p-values of $0.05,0.14,0.04,0.23$ in the four different wavelength bands from longest to shortest. This particular test accounts for shape as well as the central value of the probability distribution function, and thus some of the p-values are marginally significant. The observation at location G2, particularly in the two middle bands clearly has a substantially lower brightness temperature than the observations at location $\mathrm{C}$. The p-values for comparing the observed brightness temperatures at G2 and C are 0.05, 2e-4, 4e-5, and 3e-3 from longest to shortest wavelength, which show the difference between the $\mathrm{C}$ and $\mathrm{G}$ flux densities are strongly statistically significant except in the $10.8 \mathrm{~mm}$ band.

The marginal runs are labeled as such mostly for having intermediate weather quality. The marginal runs are shown in Figures 4.14 and 4.15. In most, the noise in each individual measurement is of the same order of magnitude as the brightness of Iapetus. Run B is an exception, as its problem is simply the small number of observations. One marginal run of note is run G1, which shows a similar pattern to that observed in run G2 at the same location, although with much greater noise (the second worst run with an actual detection of Iapetus). Runs F1 and F2 are also marginally useful runs, as their measurements are not incompatible with an intermediate value between the brightness temperatures of the pure dark and pure light equatorial regions. The plot shown in Figure 4.16 shows that F1 and F2 are detected and that their probability distribution functions (PDFs) encompass the PDFs of runs $\mathrm{C}$ and G2, thus providing a useful sanity check, although not providing any useful constraint on the steepness of the light-dark transition. 

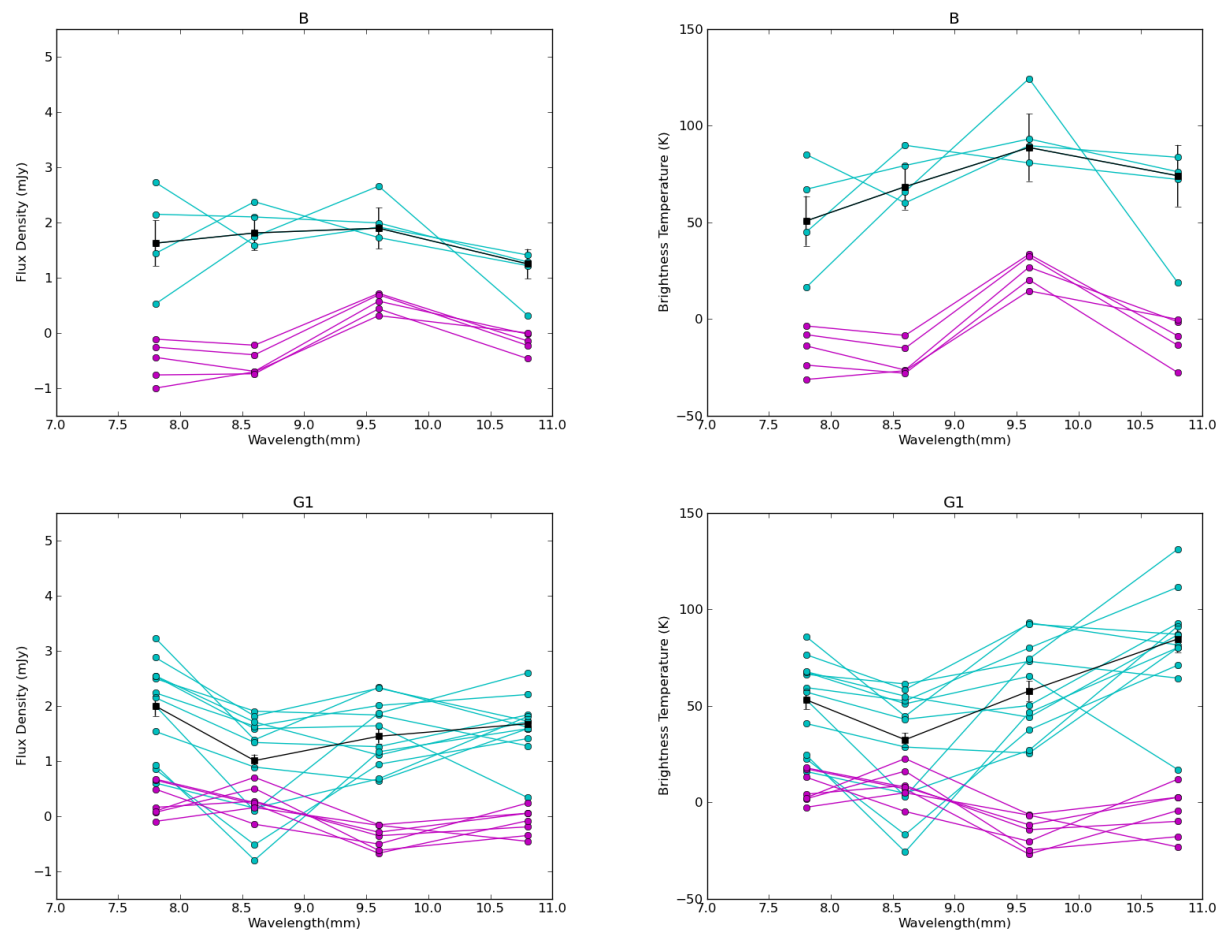

Figure 4.14: Left: Flux densities. Right: Brightness Temperatures. Two examples of marginal observing runs. Run $\mathrm{B}=$ small number of observations, although the noise for the night was quite good. Run G1 = higher noise level. While run G1 has low signal-to-noise, the pattern of observations is consistent with that seen in run G2 (Fig. 4.13) which is at an almost identical longitude. 

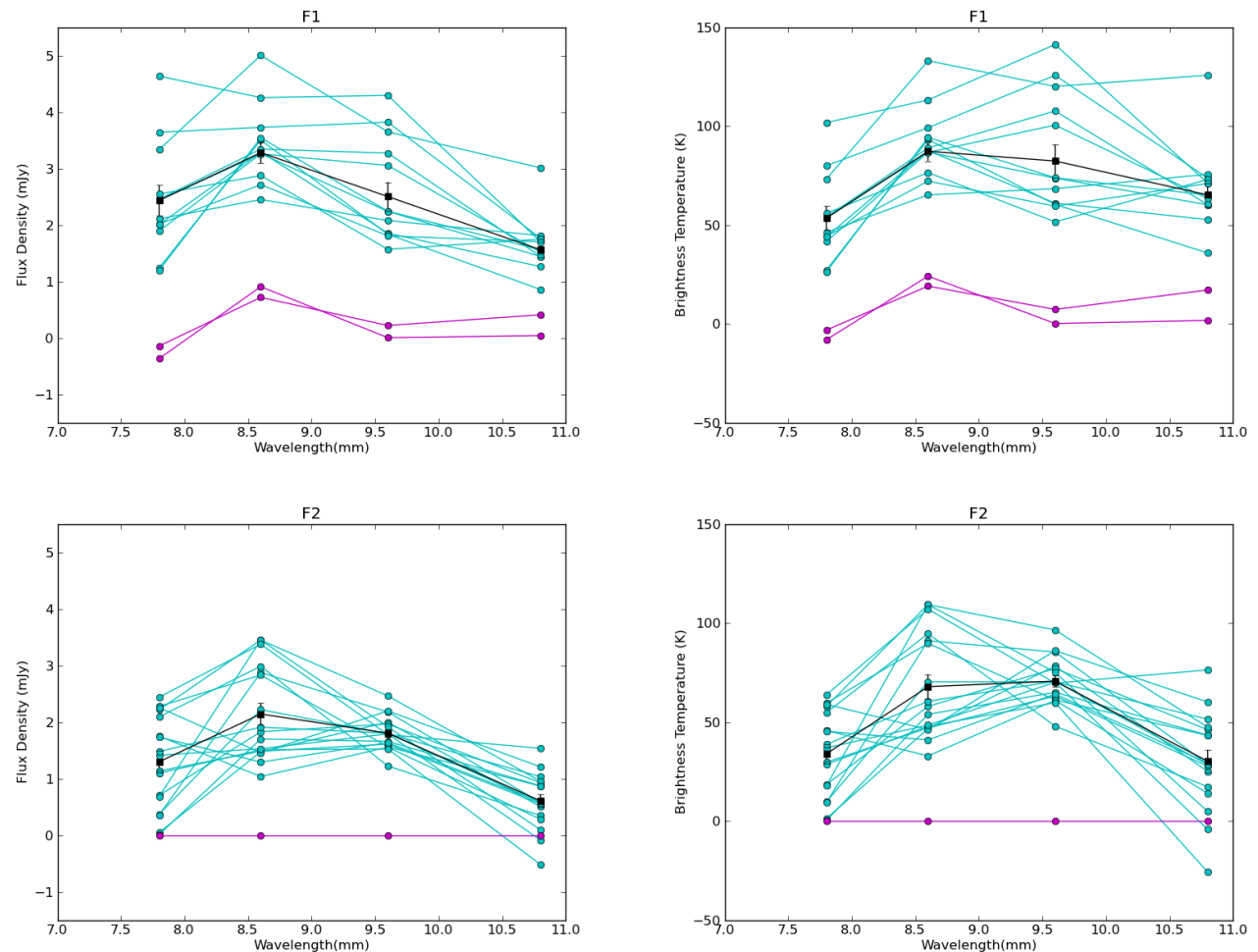

Figure 4.15: Left: Flux densities. Right: Brightness Temperatures. Two additional examples of marginal observing runs. Runs F1 and F2 are both the same longitude $\approx 225^{\circ}$ and both had low signal to noise, although run F2 has substantially more data points. 

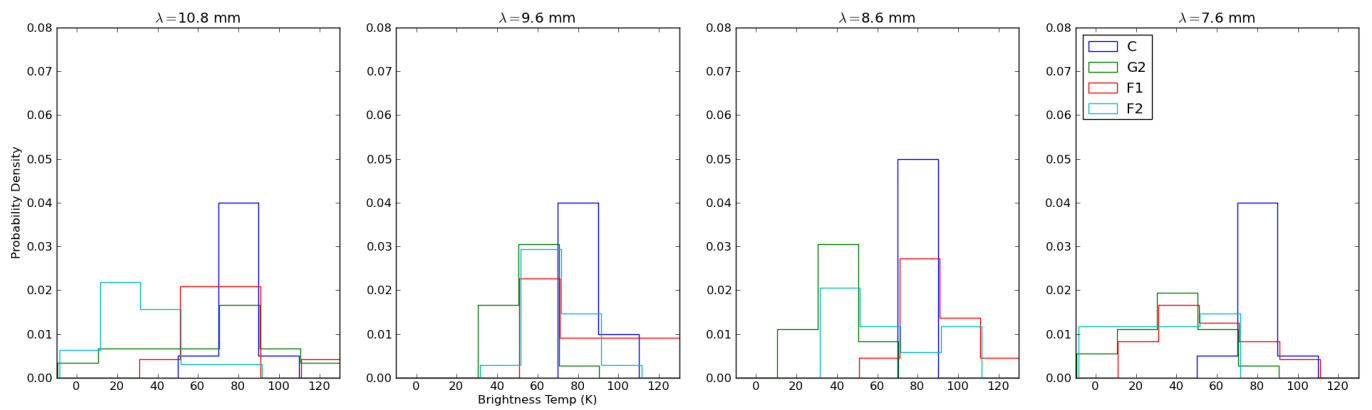

Figure 4.16: Plot of probability distributions for F1 and F2 (the only marginally successful intermediate longitude runs) as well as run C and G2 (the best all-dark and all-light material runs, respectively). The data for F1 and F2 are too noisy to make any conclusive judgments, but they both appear compatible with the values of $\mathrm{C}, \mathrm{G} 2$, and intermediate values of brightness temperature.

The failed observations are ones whose results have no relation to the physical conditions on Iapetus' surface and are shown in Figure 4.17 and 4.18. An attempted observation of Iapetus at its closest approach to Saturn (position A) failed spectacularly due to the presence of sidelobe contamination 10x brighter than Iapetus (Fig. 4.17). This result was not unexpected, given that Iapetus was less than 5 beamwidths from Saturn and that the geometry was particularly unfavorable, with Saturn and Iapetus separated almost entirely in cross-elevation. The second failed observation (position D2) was originally thought to have failed due to the fact that Saturn was only about 15 degrees from the Sun at the time of the observation. However, the main cause turned out to be that run D2 had the 3rd worst weather of any of the observing runs, as seen in Table 4.11, while Saturn was also near its farthest distance from Earth. In addition, as discussed in the previous section, sidelobe contamination may have also contributed.

The latter two failed observations (Fig. 4.18) were both at the $\mathrm{H}$ location (runs $\mathrm{H} 1$ and H2). This failure is unfortunate, as the only MUSTANG observation of the 

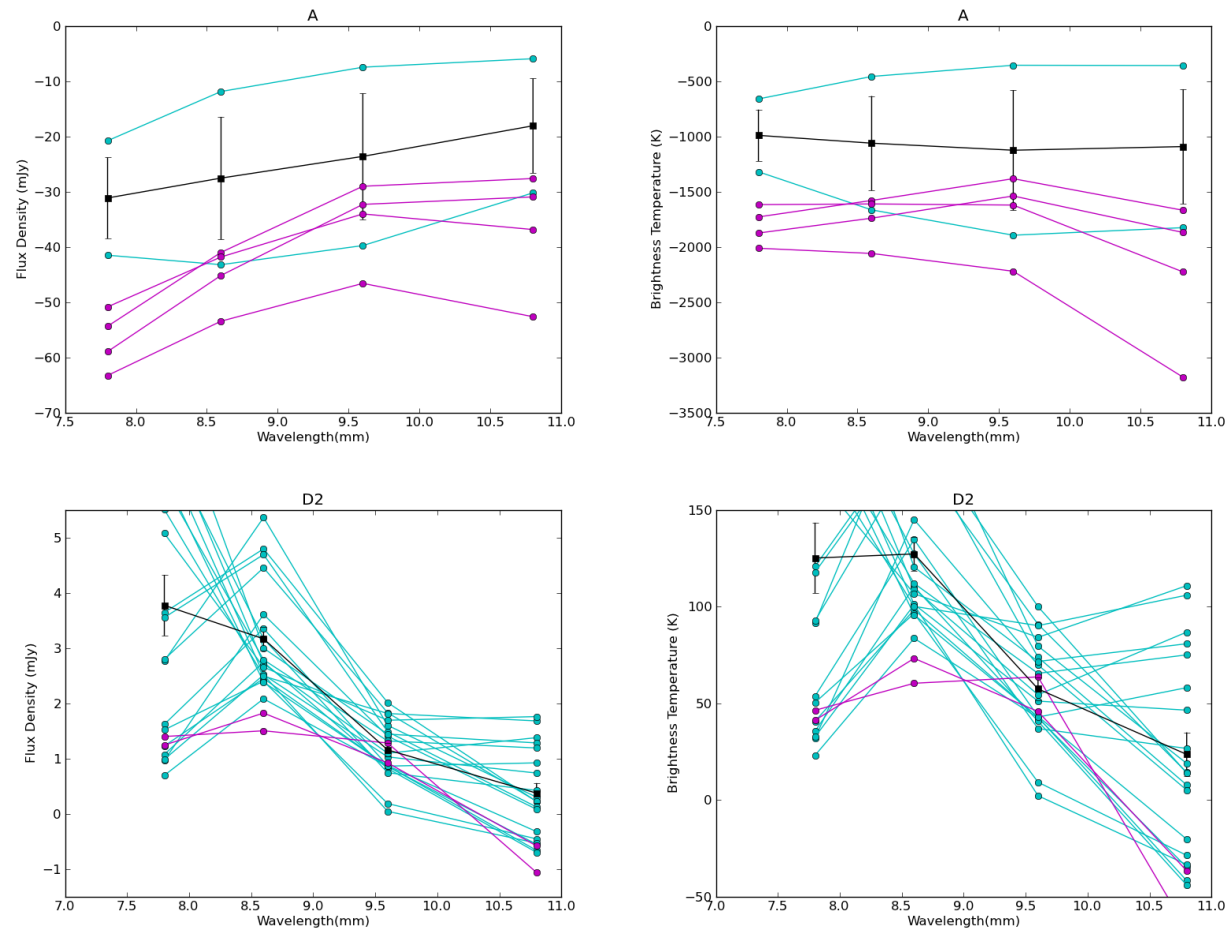

Figure 4.17: Left: Flux densities. Right: Brightness Temperatures. Observations that failed to produce any usable science. Run $\mathrm{A}=$ extreme sidelobe contamination. Run D2=more subtle sidelobe contamination.

trailing hemisphere was also at this location. In the case of run H1, the instrument suffered a major malfunction which resulted in meaningless data. In the case of run $\mathrm{H} 2$, the weather was exceedingly poor and thus Iapetus was not detected at all. Run H2 provides a good example of what a non-detection looks like, however.

\section{5 $3 \mathrm{~mm}$ Observational Results}

In terms of the MUSTANG results, the two observations show that the optically dark material is microwave bright $(\approx 100 \mathrm{~K})$ whereas the optically bright material is relatively dim (only $65 \mathrm{~K}$ ) at $3.3 \mathrm{~mm}$. As mentioned earlier, the calibration of the bright 

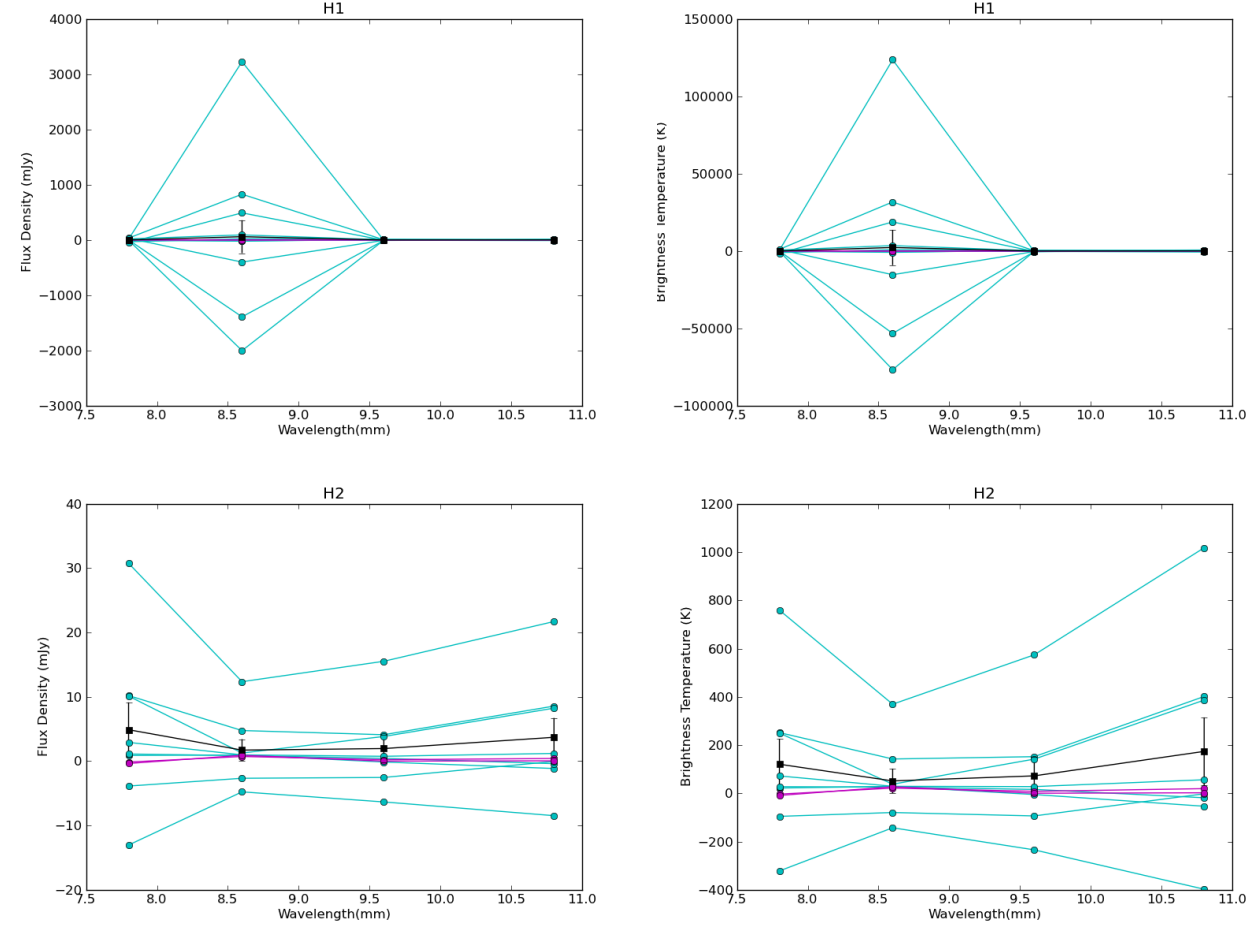

Figure 4.18: Left: Flux densities. Right: Brightness Temperatures. More observations that failed to produce usable science, both at location $\mathrm{H}\left(315^{\circ}\right.$ longitude). Run $\mathrm{H} 1=$ instrument failure. Run $\mathrm{H} 2=$ Bad weather/non-detection 


\begin{tabular}{|c|c|c|c|c|}
\hline $\begin{array}{c}\text { Obs. Longitude } \\
{ }^{\circ} \text { (run\#) }\end{array}$ & $\begin{array}{c}10.8 \mathrm{~mm} \mathrm{~S} \mathrm{~S}_{\nu} \\
(\mathrm{mJy})\end{array}$ & $\begin{array}{c}9.6 \mathrm{~mm} \mathrm{~S}_{\nu} \\
(\mathrm{mJy})\end{array}$ & $\begin{array}{c}8.6 \mathrm{~mm} \mathrm{~S}_{\nu} \\
(\mathrm{mJy})\end{array}$ & $\begin{array}{c}7.8 \mathrm{~mm} \mathrm{~S}_{\nu} \\
(\mathrm{mJy})\end{array}$ \\
\hline $41(2)$ & $1.25 \pm 0.27$ & $1.89 \pm 0.37$ & $1.81 \pm 0.31$ & $1.62 \pm 0.41$ \\
\hline $103(7)^{*}$ & $1.77 \pm 0.05$ & $2.34 \pm 0.05$ & $2.84 \pm 0.05$ & $3.57 \pm 0.08$ \\
\hline $125(3)^{*}$ & $1.04 \pm 0.22$ & $1.64 \pm 0.16$ & $1.79 \pm 0.13$ & $2.78 \pm 0.25$ \\
\hline $218(6)$ & $1.56 \pm 0.14$ & $2.50 \pm 0.26$ & $3.28 \pm 0.19$ & $2.43 \pm 0.27$ \\
\hline $226(11)$ & $0.61 \pm 0.11$ & $1.80 \pm 0.07$ & $2.14 \pm 0.19$ & $1.30 \pm 0.19$ \\
\hline $265(9)^{*}$ & $0.94 \pm 0.28$ & $1.13 \pm 0.13$ & $1.06 \pm 0.11$ & $1.25 \pm 0.13$ \\
\hline $282(4)$ & $1.67 \pm 0.13$ & $1.44 \pm 0.13$ & $1.01 \pm 0.11$ & $2.00 \pm 0.18$ \\
\hline $\begin{array}{c}\text { Obs. Longitude } \\
\quad \text { o(run\#) }\end{array}$ & $\begin{array}{c}10.8 \mathrm{~mm} \mathrm{~T} \mathrm{~T}_{b} \\
(\mathrm{~K})\end{array}$ & $\begin{array}{c}9.6 \mathrm{~mm} \mathrm{~T}_{(\mathrm{K})} \\
\mathrm{T}_{b}\end{array}$ & $\begin{array}{c}8.6 \mathrm{~mm} \mathrm{~T}_{(\mathrm{K})} \\
\mathrm{T}_{b}\end{array}$ & $\begin{array}{c}7.8 \mathrm{~mm} \mathrm{~T} \mathrm{~T}_{b} \\
(\mathrm{~K})\end{array}$ \\
\hline $41(2)$ & $74.1 \pm 15.9$ & $88.7 \pm 17.4$ & $68.4 \pm 11.8$ & $50.7 \pm 12.9$ \\
\hline $103(7)^{*}$ & $75.7 \pm 2.00$ & $80.1 \pm 1.64$ & $77.4 \pm 1.38$ & $80.4 \pm 1.83$ \\
\hline $125(3)^{*}$ & $58.7 \pm 12.5$ & $72.7 \pm 7.1$ & $64.2 \pm 4.8$ & $82.4 \pm 7.5$ \\
\hline $218(6)$ & $65.2 \pm 5.82$ & $82.4 \pm 8.4$ & $87.3 \pm 5.0$ & $53.5 \pm 6.02$ \\
\hline $226(11)$ & $30.3 \pm 5.82$ & $70.7 \pm 2.75$ & $67.9 \pm 6.21$ & $34.0 \pm 5.07$ \\
\hline $265(9)^{*}$ & $59.0 \pm 17.1$ & $54.8 \pm 6.30$ & $41.3 \pm 4.21$ & $40.3 \pm 4.41$ \\
\hline $282(4)$ & $84.6 \pm 6.89$ & $57.7 \pm 5.19$ & $32.4 \pm 3.57$ & $53.1 \pm 4.89$ \\
\hline
\end{tabular}

Table 4.12: List of all marginal or good observations of Iapetus taken over the course of this project. All brightness temperatures are derived based on a radius of 735.6 $\mathrm{km}$ (Thomas et al. 2007). Uncertainties given are error of the mean for measured flux densities and exclude calibration and other systematic uncertainties. ${ }^{*}=$ Best weather. Note that "best weather" is not correlated with lowest errors, since the magnitude of the errors is also a function of number of observations on each night. Table 4.11 gives the measurement noise for individual observations on each night. 


\begin{tabular}{|l|c|c|}
\hline Phase & $\begin{array}{c}\mathrm{S}_{\nu} \\
(\mathrm{mJy})\end{array}$ & $\begin{array}{c}\mathrm{T}_{b} \\
(\mathrm{~K})\end{array}$ \\
\hline $\mathrm{C}$ & $23.1 \pm 1.0$ & $97.4 \pm 4.0$ \\
$\mathrm{H}$ & $16.5 \pm 2.5$ & $64 \pm 10.0$ \\
\hline
\end{tabular}

Table 4.13: MUSTANG observations of Iapetus. Note that H observation uncertainty given is not measurement uncertainty, but rather uncertainty in the calibration, which is substantially larger.

material observation was less than ideal, as no standard calibrators were observed. Using Vesta, the better of the two calibrators, results in a measured $\mathrm{T}_{b}$ of around 55 $\mathrm{K}$. There is also an approximately $10 \%$ uncertainty in the measured $\mathrm{T}_{b}$ owing to measurement noise. Unfortunately, for logistical reasons, no direct observation of Iapetus' trailing hemisphere (location G) was performed with MUSTANG, so some modeling is required to extrapolate the appropriate brightness temperature from point $\mathrm{H}$ to compare with $\mathrm{CCB}$ observations at point $\mathrm{G}$, and both attempted $\mathrm{CCB}$ observations at point $\mathrm{H}$ failed (instrument failure, inadequate signal-to-noise). 


\section{Chapter 5}

\section{Modeling}

\section{$5.1 \quad$ Modeling}

\subsubsection{Thermal Modeling}

In order to relate these results to $\epsilon_{\text {eff }}$, I first had to calculate a physical surface temperature. I calculated this temperature using data obtained from the Cassini mission in the infrared (Carvano et al. 2007; Howett et al. 2010). ${ }^{1}$ Rather than use an approximation such as the beaming factor, $\eta$ used in the STM (Spencer et al. 1989), this model explicitly modeled the surface temperature distribution over time. I created a model of the surface of Iapetus that took as input the sun's position over Iapetus and the observer's position over Iapetus and returned a snapshot in time of the temperature distribution across Iapetus seen by the observer. An average surface temperature of Iapetus for that observer was then calculated. Figure 5.1 shows an

\footnotetext{
${ }^{1}$ The model of Rivera-Valentin et al. (2011) was not used for this calculation, as that model derived the minimum, mean, and maximum temperatures for each location on Iapetus. For this work, the biggest concern was modeling the temperature distribution at a given time, which is better modeled by Howett et al. (2010). However, the results of my model for minimum and maximum temperatures show reasonable agreement with the values of Rivera-Valentin et al. (2011), with the main difference being that their model is slightly colder in the polar regions.
} 
example of this solar heating model. This model is not a physical one, but rather an empirical one which attempts to replicate the observed temperatures on Iapetus' surface rather than constrain the underlying physical properties of Iapetus' surface.

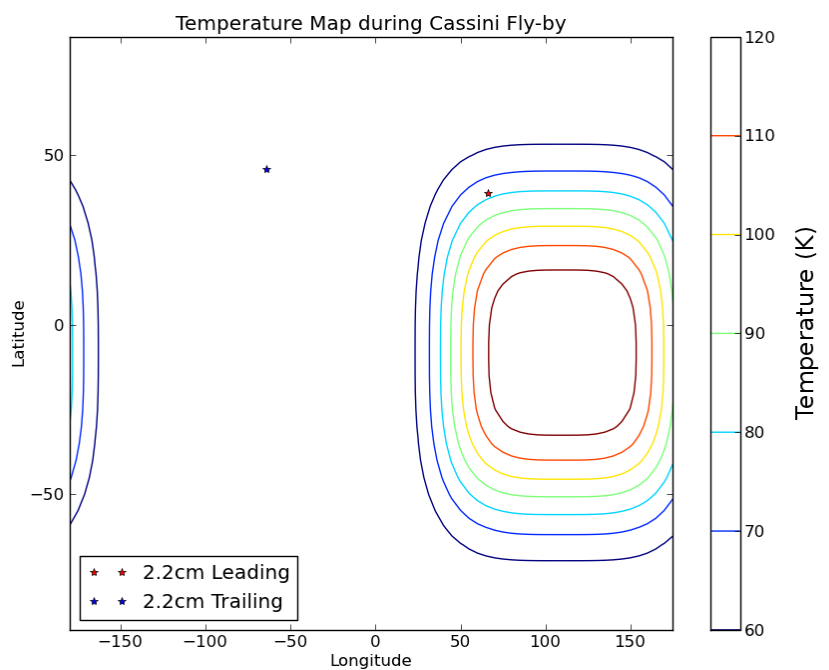

Figure 5.1: Plot of an example thermal map of Iapetus' surface that replicates the surface temperature of Iapetus during the Cassini flyby of $12 / 31 / 2004-1 / 1 / 2005$. Also marked on the map are the locations of RADAR observations made by the Cassini spacecraft during that flyby. The rotation of Iapetus is slow, with the sub-solar longitude changing only by $10^{\circ}$ over the course of the flyby.

The temperature model was then projected onto the disk facing the observer, and adjusted for a resolved surface when necessary (Cassini RADAR data). Example projected disks are shown in Figure 5.2 for both this thermal model and the three-tone model described below.

The main result of the model is that despite the fact that Iapetus' equatorial region can vary in temperature by up to $10 \mathrm{~K}$, the average temperature of the surface as seen from Earth only shows about a $1 \mathrm{~K}$ variation and averages approximately $100 \mathrm{~K}$ on the Earth-facing side (see Fig. 5.3). The other important result of this model is that the 

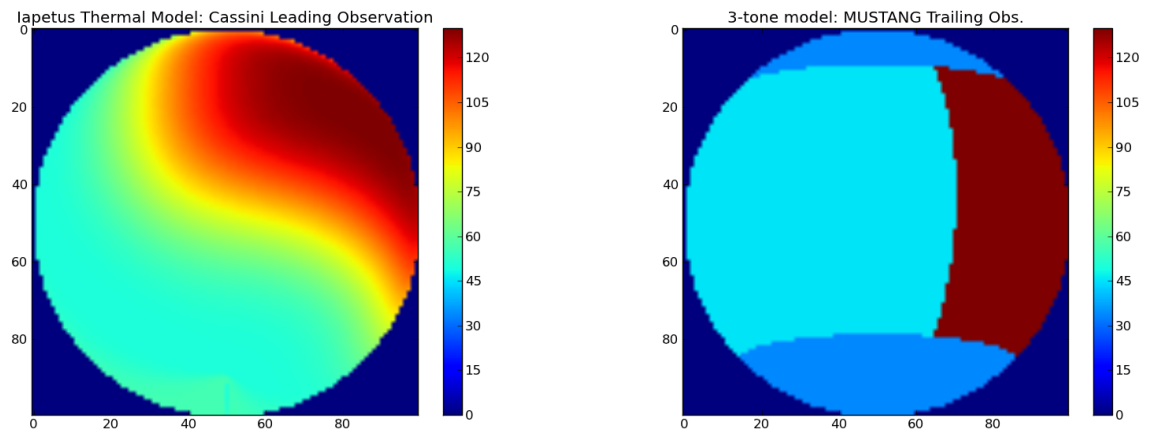

Figure 5.2: Plots of thermal models projected on to the observed disk of Iapetus. The figure on the left is a projection of the thermal model of the disk as seen by Cassini during its observation of the dark leading hemisphere of Iapetus and the figure on the right is a projection of the three-tone model of Iapetus as seen by MUSTANG during observing run $\mathrm{Gm}$ in the intermediate region with some dark and some light material.

night side of Iapetus shows a nearly-uniform temperature of approximately 60K. I then used this data to calculate $\epsilon_{\text {eff }}$ for my data from 3-11 $\mathrm{mm}$ and additional radiometric data obtained on Iapetus from Cassini with its RADAR instrument operating at 22 $\mathrm{mm}$ (Ostro et al. 2006), which is discussed below in the section on emission models.

\subsubsection{Three-tone modeling}

In order to to obtain the $3.3 \mathrm{~mm}$ brightness temperature of Iapetus' trailing hemisphere, I had to derive a realistic model for the brightness temperature of Iapetus in order to translate the observations at location $\mathrm{H}$ (an intermediate region) and location $\mathrm{C}$ (the middle of the dark equatorial region) into a $\mathrm{T}_{b}$ for location $\mathrm{G}$ (the middle of the bright equatorial region). Iapetus effectively consists of three regions: a bright polar region, a bright equatorial region, and a dark equatorial region. For my model, I used a $\mathrm{T}_{b}$ of $40 \mathrm{~K}$ for the bright equatorial region, $115 \mathrm{~K}$ for the dark equatorial region, and 30K for the polar region. Figure 5.4 shows this model. Using this model, 


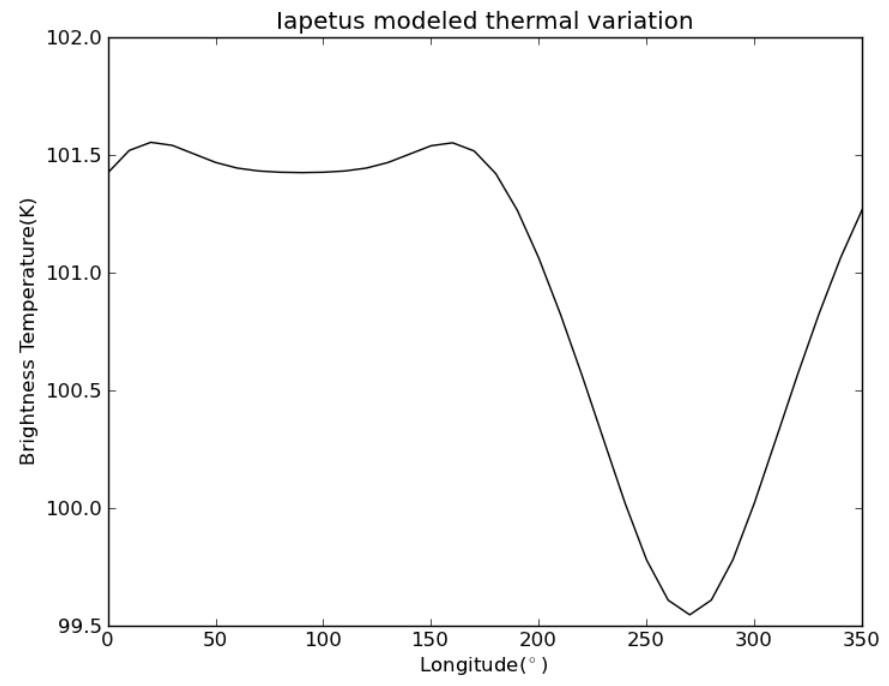

Figure 5.3: Iapetus' thermal light curve at the sub-solar and sub-observer $12^{\circ}$ of latitude.

I computed that $98 \mathrm{~K}$ would be the expected brightness temperature at point $\mathrm{C}, 58 \mathrm{~K}$ at point $\mathrm{H}$, and $42 \mathrm{~K}$ at point $\mathrm{G}$. The $\mathrm{C}$ and $\mathrm{H}$ points used in this model match my MUSTANG $(\mathrm{H}=55 \mathrm{~K}, \mathrm{C}=97.4 \mathrm{~K})$ data well.
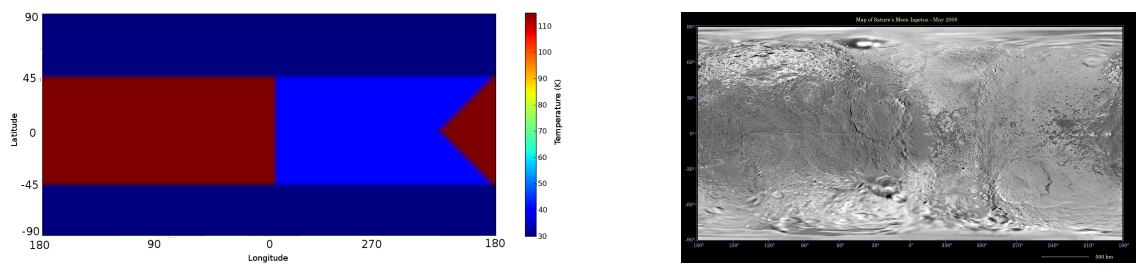

Figure 5.4: Plot of the three-tone model used to interpolate MUSTANG data. Left: Model. Right: Visible Map of Surface from Voyager (Smith et al. 1982) and Cassini (Porco et al. 2005) probes. Image Credit:NASA/JPL/Space Science Institute. 


\subsubsection{SED modeling}

\section{Dark Leading Hemisphere}

So what physical mechanism is responsible for the variations in $\epsilon_{\mathrm{eff}}$ ? As detailed in the introduction, the two most common explanations for deviation from an $\epsilon_{\text {eff }}$ of 1.0 for astronomical bodies were given in Redman et al. (1992): thermal depth effect and scattering. The thermal depth effect works as follows. For a slow-rotating moon like Iapetus, the thermal skin depth on Iapetus should be approximately $10 \mathrm{~m}$ assuming a $100 \mathrm{~K}$ surface of pure water ice using the equations previously outlined in the introduction. The expected depth probed by $1 \mathrm{~cm}$ measurements is of order tens of $\mathrm{cm}$, and thus should therefore not show a thermal depth effect, using the 10-30 $\lambda$ rule of thumb. However, a more explicit calculation can be made by using the dielectric constant for water and the surface model of Muhleman (1972). Muhleman (1972) derived the radio absorption length of a surface as:

$$
l_{\mathrm{R}, \nu}=\frac{\lambda}{2 \pi \sqrt{\epsilon_{\mathrm{d}}} \tan \Delta}
$$

where $\epsilon_{\mathrm{d}}$ is the dielectric constant, $\tan \Delta$ is the loss tangent, and $\lambda$ is wavelength. Essentially, the denominator of this equation provides a multiplier for wavelength. The dielectric constant of water ice at 100K is approximately 160 (Hobbs et al. 1966) and the loss tangent is 0.0014 in glacier ice (Chang et al. 1976). The resulting radio skin depth is therefore $9 \lambda$, so about $2.7 \mathrm{~cm}$ at $\lambda=3.3 \mathrm{~mm}$ and about $9 \mathrm{~cm}$ at $\lambda=1 \mathrm{~cm}$ for a surface of pure water ice. Therefore, we should not be able to detect a thermal depth effect with our observations, if the surface is pure water ice. However, the upper surface of Iapetus' dark Cassini Regio is mostly a contaminant material, identified as tholin or hematite (Clark et al. 2012). For hematite, the thermal depth is expected to 
be more like $45 \mathrm{~cm}$ (using $C_{p}=40$ (extrapolated from (Jayasuriya et al. 1985) to $100 \mathrm{~K}$ temperature), $K_{T}=12.42 \mathrm{~W} \mathrm{~m}^{-1} \mathrm{~K}^{-1}$ (Clauser and Huenges 1995) and $\rho=5300 \mathrm{~kg}$ $\mathrm{m}^{-3}{ }^{2}$ ), which should lead to detection of a thermal depth effect at CCB wavelengths assuming a 10-30 $\lambda$ penetration depth. ${ }^{3}$

A thermal depth effect appears to be exactly what is going on in terms of Iapetus' dark, leading hemisphere. Figure 5.5 shows the combination of observations taken with MUSTANG, the CCB, and the RADAR instrument on Cassini (Ostro et al. 2006) which show a fairly shallow decline from almost precisely the surface temperature $(100 \mathrm{~K})$ at $3 \mathrm{~mm}$ to about $73 \mathrm{~K}$ at $2.2 \mathrm{~cm}$ as well as a plot of a thermal depth curve using the thermal length of pure hematite and $20 \lambda$ penetration depth. Given the sharp change between the MUSTANG and CCB data points, this model is clearly not adequate, requiring a sharper temperature difference between the surface and subsurface and also possibly caused by a smaller than expected penetration depth, a change in $\epsilon$ with $\lambda$, or a lower value of $K_{T}$ than that of pure hematite.

Figure 5.6 gives a broader perspective of the emission spectrum of Iapetus, combining the microwave data with infrared data obtained from the CIRS instrument from 25-200 $\mu \mathrm{m}$ (Carvano et al. 2007), which shows no deviation from a blackbody. The data from this work fills a large gap in wavelength coverage between the RADAR observations (Ostro et al. 2006) and the CIRS observations, approximately half-way between the two existing data sets in log space.

\footnotetext{
${ }^{2}$ Source: Wolfram Alpha http://www.wolframalpha.com

${ }^{3}$ Pure hematite is a microwave insulator, so its absorption length may be as short as a fraction of a wavelength (Ostro et al. 2006). $\epsilon_{\mathrm{d}}$ for hematite is 87.0, and the loss tangent is 0.07 when derived from (Tikhonov et al. 2010), which yields a penetration depth of .03 $\lambda$, however this value does not account for other contaminants.
} 


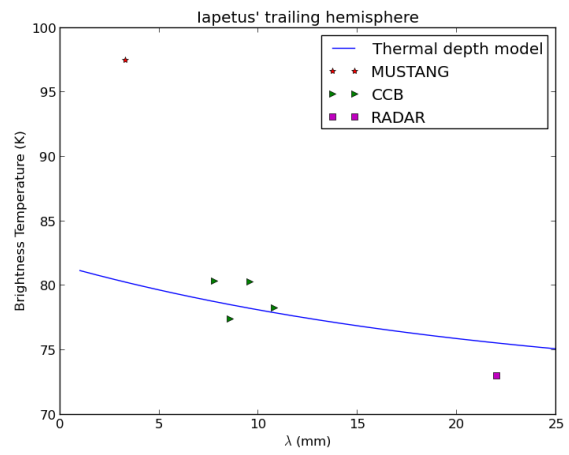

Figure 5.5: Brightness temperature of the leading hemisphere of Iapetus showing a shallow decline as wavelength increases, consistent with a thermal depth effect. The model curve has $T_{\text {surf }}=102.0 \mathrm{~K}$ (from thermal model), $T_{\text {eq }}=89.9 \mathrm{~K}$ (Ostro et al. 2006), $\epsilon=0.8$ (Ostro et al. 2006), $L_{T}=45 \mathrm{~cm}$ (the calculated value for pure hematite on Iapetus), and penetration depth $20 \lambda$ (approximation).

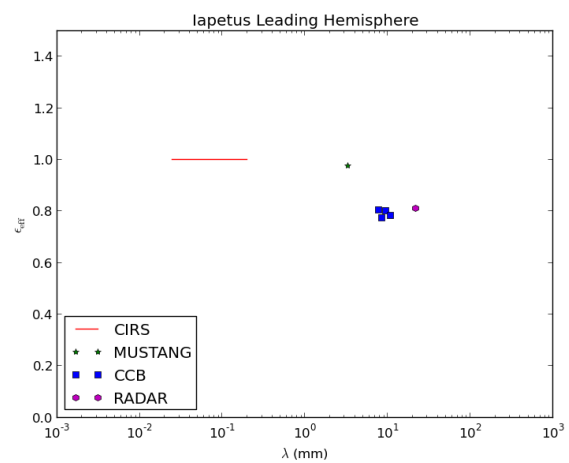

Figure 5.6: Effective emissivity vs. wavelength including data from the CIRS instrument (Carvano et al. 2007) and the original emissivity from (Ostro et al. 2006). 


\section{Bright Trailing Hemisphere (Roncevaux Terra)}

The scattering model of Redman et al. (1992) produces a U-shaped drop in brightness temperature over two orders of magnitude in wavelength in asteroid 4 Vesta. Unfortunately, their model cannot account for the trailing hemisphere observations of Iapetus, as the drop in brightness temperature in the $1 \mathrm{~cm}$ region is too rapid (drop of $30 \%$ or more in less than a factor of two in wavelength). However, such drops in brightness temperature have been observed previously on Earth. A substantial amount of research has been done at wavelengths ranging from a bit less than $2 \mathrm{~mm}$ to as long as $10 \mathrm{~cm}$ on icy surfaces here on Earth, as these wavelengths correspond to the bands of receivers in the Microwave Sounding Units (MSUs) and Advanced Microwave Sounding Units (AMSUs) (Vangasse et al. 1996) used on NOAA satellites. In fact, bands $16(89 \mathrm{GHz})$ and $2(31.4 \mathrm{GHz})$ of the AMSUs correspond almost precisely to my observations with MUSTANG and the CCB, respectively. These sensors are used in climate research to measure the Earth's global surface and atmospheric temperature as well as numerous other important climate variables. Observations by aircraft and satellites have revealed some similar drops in brightness temperature on Earth (Hewison et al. 2002; Weng et al. 2001; Harlow 2007) to those seen on Iapetus in the bright Roncevaux Terra.

Of particular interest is the terrestrial snowpack model of Wiesmann and Mätzler (1999) called the Microwave Emission Model for Layered Snowpacks (MEMLS) which takes as input various physical parameters of snow(depth of scattering layer, temperature, density, grain size) and produces brightness temperatures from $3 \mathrm{~mm}$ to several $\mathrm{cm}$ in wavelength. The model is a semi-empirical model which uses a combination of experimental data and theory. In particular, the scattering within and between layers is based on laboratory measurements performed on Earth rather than various 
scattering theories. The model uses the theoretical framework of the two-stream approximation to calculate radiative transfer. Is this model relevant for the celestial as well as the terrestrial? There are some approximations made which are linear with respect to temperature, however these linearizations should hold so long as the temperature is significantly greater than absolute zero, which is true even for a satellite as cold as Iapetus. The model is also relatively limited in terms of grain sizes (100$3000 \mu \mathrm{m})$, whereas astrophysical grain sizes can range anywhere from hundreds of $\mathrm{nm}$ to tens of $\mathrm{cm}$. These limitations are therefore worth keeping in mind, but not severe enough to rule out the model being relevant to Iapetus.

I ran numerous models varying grain size, depth of scattering layer, volume fraction, subsurface temperature. The left half of Figure 5.7 shows a fit of MEMLS to my data on the trailing hemisphere from MUSTANG and the CCB, and the data from Cassini. While the slope of the CCB data is well-fit, neither the Cassini nor MUSTANG data are. However, there are reasons that both data points should be driven towards something resembling this model. First, as mentioned earlier, the MUSTANG observation of the trailing hemisphere was made in an intermediate region, rather than just bright material. Applying the three-tone model above reduces the brightness temperature from $65 \mathrm{~K}$ to $42 \mathrm{~K}$ and brings the curve to within the error bars on the data.

The second problem is with the Cassini data, which has a similar problem to the MUSTANG data of not-comparable observing conditions. Unlike when observing from Earth, Cassini can sometimes observe the night side of moons in the Saturn system. It turns out that the trailing RADAR observation was made deep in the night side of Iapetus, where, as mentioned in the thermal modeling section, the physical surface temperature is $60 \mathrm{~K}$. The measured brightness temperature for the trailing 

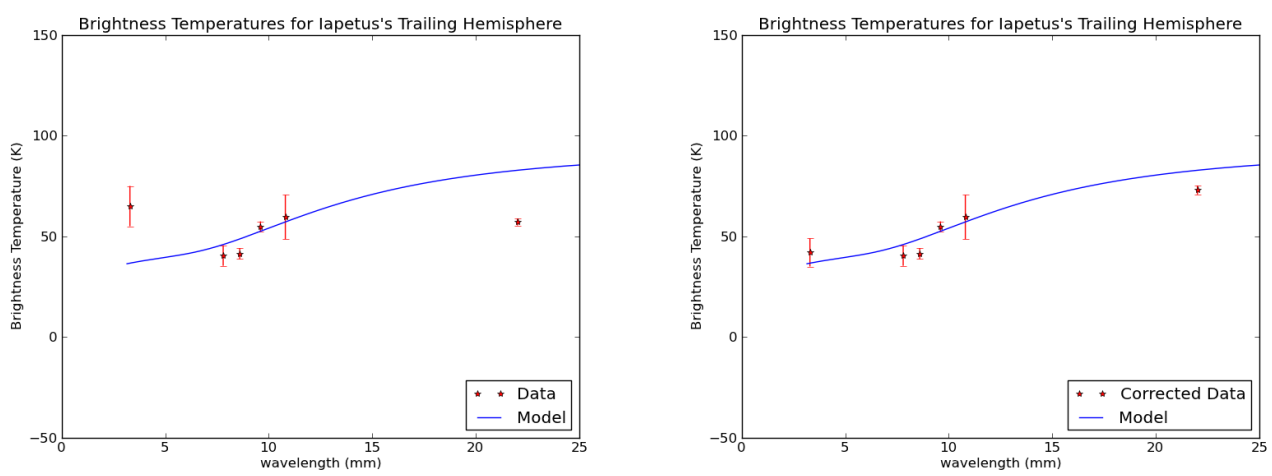

Figure 5.7: Left.) Brightness temperature of the trailing hemisphere of Iapetus showing steep incline in brightness temperature around $1 \mathrm{~cm}$ with original data points for $2.2 \mathrm{~cm}$ and $3.3 \mathrm{~mm}$ and MEMLS model. Right.) After applying corrections to MUSTANG data to compensate for change in longitude and Cassini data to compensate for observation of night side which provide a much better fit to the data.

side at $2.2 \mathrm{~cm}(57 \mathrm{~K})$, which implies an $\epsilon_{\text {eff }}$ of approximately 1.0 (although some factors could lead to a modestly lower value). The calculated changes in effective emissivity are given in table 5.1. Given that the daytime surface temperature on the the trailing side should be approximately $100 \mathrm{~K}$, a $2.2 \mathrm{~cm}$ brightness temperature of $80 \mathrm{~K}$ is entirely plausible, if not one even higher, given $\epsilon_{\mathrm{eff}}$ of 1.0 at $2.2 \mathrm{~cm}$. At the very minimum, the daytime trailing brightness temperature at $2.2 \mathrm{~cm}$ should be at least as great as the daytime leading hemisphere at that same wavelength. The right half of Figure 5.7 shows this and the MUSTANG observation corrected via the threetone model described above. This model was derived by searching for the best fit to the corrected data in parameter space, and an example of the search in scattering layer depth and grain size is shown in Figure 5.8. The model used has an isothermal surface of temperature $100 \mathrm{~K}$, a volume fraction of 0.8 , a grain size of $1-2 \mathrm{~mm}$, and a scattering layer of depth $\approx 1 \mathrm{~m}$.

Regarding polarization, the MEMLS model actually produces the expected brightness temperature in both linear polarizations, rather than just the total. When ob- 


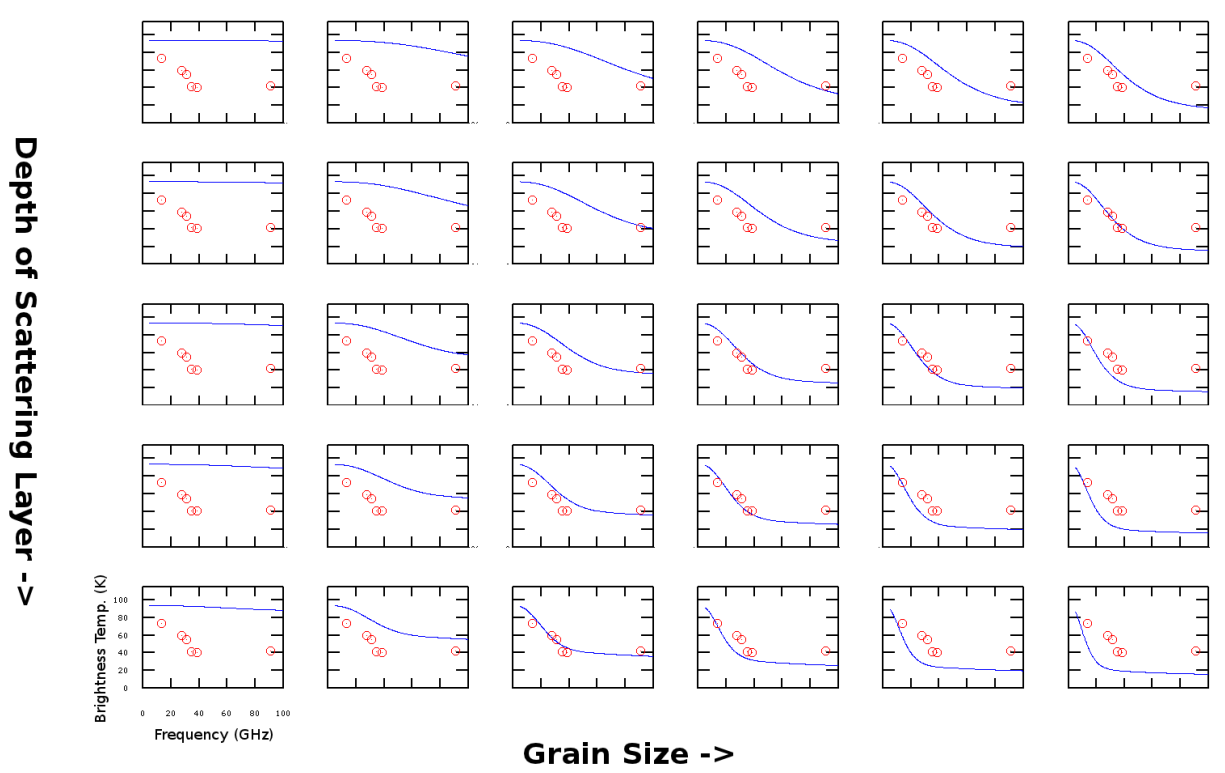

Figure 5.8: Attempting to fit the steepness of the $1 \mathrm{~cm}$ decline in brightness temperature with variants of the MEMLS model. Depth of scattering layer increases down and grain size increases to the right. The model chosen was the bottom row, third from the left, which was unequivocally the best choice.

\begin{tabular}{|c|c|c|c|c|c|c|}
\hline Side & $\begin{array}{c}2.2 \mathrm{~cm} \mathrm{\textrm {T } _ { b }} \\
(\mathrm{K})\end{array}$ & $\begin{array}{c}\mathrm{T}_{\text {ref,orig }} \\
(\mathrm{K})\end{array}$ & $\epsilon_{\text {eff,orig }}$ & $\begin{array}{c}\mathrm{T}_{\text {ref,new }} \\
(\mathrm{K})\end{array}$ & $\epsilon_{\text {eff,new }}$ & SL Albedo \\
\hline Leading & $73.0 \pm 2.2$ & 89.9 & 0.81 & 80.27 & 0.91 & 0.31 \\
Trailing & $57.1 \pm 1.7$ & 83.3 & 0.69 & 53.6 & 1.06 & 0.57 \\
\hline
\end{tabular}

Table 5.1: Results of re-calculating the physical temperature used for determining emissivity from Ostro et al. (2006). Ostro et al. (2006) used an equilibrium temperature, however, this re-calculation uses surface temperatures calculated from infrared observations of Iapetus (Howett et al. 2010). SL (scattering law) albedo results from the same are also listed for convenience. 
serving straight down upon a surface, the emission from the surface is completely unpolarized in the MEMLS model, regardless of what other input parameters are chosen. However, when observing at an angle, the emission can become substantially polarized. Nonetheless, the average brightness temperature and total flux density are unaffected by incidence angle in the MEMLS model. Thus brightness temperatures derived from CCB beam-switching nod observations, which measure the average of the two linear polarizations, should not be affected by this predicted polarization when making comparisons with the total brightness temperature produced by the MEMLS model.

In the context of previous observations, Figure 5.9 shows all of the observations of Iapetus' trailing hemisphere including from CIRS and the corrected MUSTANG (with the three-tone model) and RADAR observations (accounting for night-side observing). The result is a sharp and deep absorption feature centered near $3 \mathrm{~mm}$. Future observations in the 1-3 mm range with ALMA or the SMA will likely reveal interme-

diate emissivities and the short wavelength end of the absorption trough caused by scattering in the ice. While MEMLS cuts of at $100 \mathrm{GHz}$, other Earth microwave observations show that most forms of ice return to near unity $\epsilon_{\text {eff }}$ at shorter wavelengths (Hewison et al. 2002).

\subsection{Science Conclusions}

\subsubsection{Variations in Effective Emissivity}

Iapetus shows tremendous variation in $\epsilon_{\text {eff }}$ with both longitude and wavelength between 3 and $11 \mathrm{~mm}$. These observations filled an important gap in existing wavelength coverage of Iapetus. The detected variation can be fit with a thermal depth effect on 


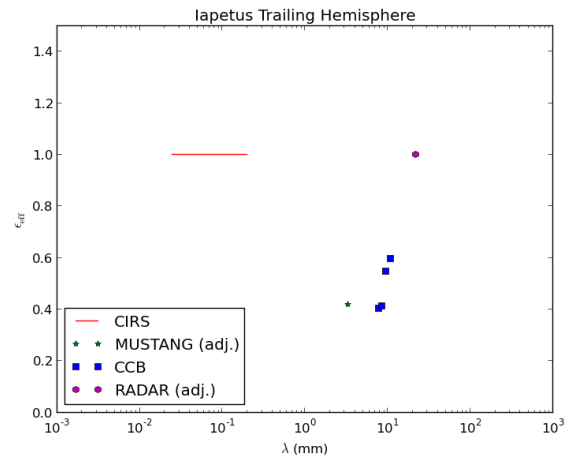

Figure 5.9: Effective emissivity vs. wavelength for the bright trailing hemisphere of Iapetus (Roncevaux Terra/Saragossa Terra) including data from the CIRS instrument (Carvano et al. 2007) and the updated emissivity from (Ostro et al. 2006).

the leading side and with a water-ice scattering model on the trailing side.

The water-ice scattering is especially interesting thanks to the upcoming completion of the Atacama Large Millimeter Array (ALMA). ALMA will be the most sensitive telescope for the detection of thermal emission from of trans-Neptunian objects (TNOs), as can be seen in Figure 5.10. TNOs are astrophysically interesting objects, as they are an important clue in understanding the formation of the solar system. Of particular relevance to work with ALMA is a break that has been detected in the optical size distribution of the Kuiper Belt (Fraser and Kavelaars 2009). It is unclear if this break corresponds to albedo or size, but this break is important in determining the collisional history of the solar system(Kenyon et al. 2008). Only a few tens of TNOs have had sizes determined from thermal emission in the IR(Stansberry et al. 2008), although more than 100 will be detected as part of the "TNOs are Cool" program with Herschel (Müller et al. 2010). Neither Herschel nor Spitzer has the sensitivity to reach the break in the magnitude distribution, but ALMA will(Moullet et al. 2011).

Water ice is common on the surface of TNOs. As a result of the complications 


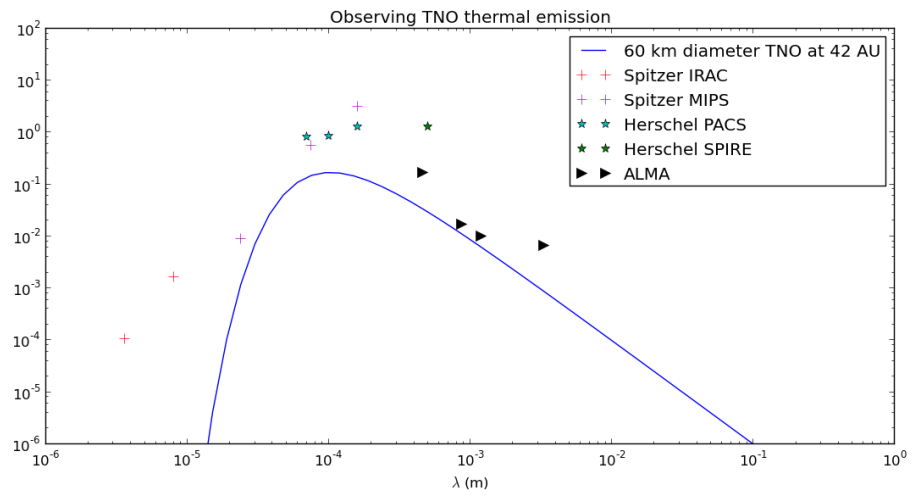

Figure 5.10: Comparison of the 8 hour sensitivities of ALMA, Spitzer, and Herschel to a $60 \mathrm{~km}$ diameter TNO at $50 \mathrm{~K}, \epsilon=1.0$, and 42 AU distance for a $3 \sigma$ detection. ALMA is the best choice for studies of large numbers of TNOs, particularly in the middle ALMA bands.

encountered with water ice on Iapetus, future observers should exercise extreme caution in assuming that TNOs objects are blackbodies in the microwave and submm regions. However, such deviations from a blackbody may be useful in constraining the surface properties.

\subsubsection{Grain Size}

The exponential correlation length used for the MEMLS model on the trailing side is $0.3 \mathrm{~mm}$, which corresponds to a grain size of 1-2 mm (Wiesmann et al. 1998). Near-IR observations with the VIMS instrument on the Cassini spacecraft yield a surface grain size of 0.1-5 mm (Filacchione et al. 2012). A comparison between these two grain size derivations is important for several reasons. First, it provides validation that the water ice model of Wiesmann and Mätzler (1999) is in fact a reasonable physical model for the microwave emission of Iapetus (grains of the correct size actually exist on Iapetus). The grain size is also more tightly constrained by the microwave emissivity and is at the larger end of the grain size distribution. Both the narrower range 
and larger grain size may be a product of the subsurface being more homogeneous (i.e. narrower range of grain sizes) than the surface due to surface processes (e.g. sublimation, micrometeoroid gardening) creating a multitude of grain sizes.

Lastly, and most importantly, this observation is the first time ever that the surface or subsurface grain size has ever been constrained on an extraterrestrial body using microwave emission. This technique opens the possibility of constraining surface properties of other icy bodies (especially TNOs) which are too faint for visible or IR spectroscopy using continuum observations with ALMA, the JVLA, and eventually perhaps even the SKA.

\subsubsection{Informing earlier RADAR results}

RADAR data is complementary to brightness temperature/effective emissivity data. If there is no substantial thermal variation with depth (i.e. the effective emissivity is approximating the true emissivity), then $(1-r=\epsilon=\alpha)$, where $r$ is the albedo/reflectivity. This equation contains many important subtleties when comparing to observations. In addition to the important caveats about emissivity which have already been discussed at length, the albedo in this equation is that which is determined from bistatic radar experiments (i.e. the receiver is in a separate physical location from the emitter and ideally multiple angles of reflection are measured from the target surface). However, all of the observations of Iapetus are single scattering observations (i.e. the receiver and emitter are co-located) and thus only measure a single angle of scattering. As a result, just as $\epsilon \neq \epsilon_{\mathrm{eff}}, r \neq r_{\mathrm{SL}}$ due to various complicating factors such as the coherent backscatter effect, which allows $r_{\mathrm{SL}}$ to be as high as 4 , rather than range $0-1$ to which $\epsilon$ and $r$ are confined. In fact, RADAR observations of Titan have revealed some surface regions with $r_{\mathrm{SL}}$ of 2 which are barely fit 
by the most extreme physical models (Janssen et al. 2011).

In spite of all the subtleties, previous comparisons of Cassini RADAR albedos and emissivities have shown a linear relationship similar to Kirchhoff's law but with slightly different slope (Ostro et al. 2006). RADAR reflection experiments on Iapetus show similar results between the leading and trailing hemispheres at $10 \mathrm{~cm}$ (Black et al. 2004), but not $2 \mathrm{~cm}$ (Ostro et al. 2006) wavelengths, implying a depth of similarity of $\approx 100 \mathrm{~cm}$. My result that the depth of the scattering layer on the trailing side is $100 \mathrm{~cm}$ deep agrees with this earlier result, although it is derived via a completely different method.

However, my brightness temperature measurements at $1.1 \mathrm{~cm}$ (the longest wavelength CCB observation) are not terribly dissimilar, thus they naively imply similarity at a depth of $\approx 10 \mathrm{~cm}$. However, this similarity arises from different physical mechanisms and should not be taken to imply similar subsurface structures. The brightness temperatures on the trailing hemisphere near $1.1 \mathrm{~cm}$ are rising towards longer wavelengths owing to a decrease in scattering effect, whereas the leading hemisphere brightness temperature is declining owing to a lower physical temperature at deeper depth or the beginning of a scattering effect.

Could something similar happen in the reflection measurements with RADAR? One unexpected consequence of my thermal modeling was discovering that the Cassini RADAR reflection and brightness temperature/emissivity results at $2.2 \mathrm{~cm}$ are potentially contradictory in terms of Kirchhoff's law. The published results for leading hemisphere RADAR albedo and emissivity are 0.31 and 0.81 respectively, while for the trailing hemisphere they are 0.58 and 0.70 (Ostro et al. 2006). As expected, the two are anti-correlated. However, I recalculated $\epsilon_{\text {eff }}$ with my thermal model in Table 5.1 resulting in emissivity of $\approx 1.0$ on the trailing side and 0.91 on the leading side, 
implying that both $\epsilon$ and $r$ are greater on the trailing side. Kirchhoff's laws indicate these values should be anti-correlated, so the RADAR results now pose an intriguing mystery. Therefore, my results both confirm and question the earlier $2.2 \mathrm{~cm}$ observations. My own results provide a limit on similarity of $100 \mathrm{~cm}$ for the light and dark material, based on the depth of the scattering layer required to explain the trailing hemisphere brightness temperatures which is in agreement with that provided by $2.2 \mathrm{~cm}$ observations. However, updating the calculated RADAR emissivities during thermal modeling uncovered a discrepancy (likely caused by subtleties in $r_{\mathrm{SL}}$ and $\epsilon_{\mathrm{eff}}$ ) between the $2.2 \mathrm{~cm}$ emission and reflection which merits a more detailed investigation.

\subsubsection{Future work}

In the immediate future, I have already observed six other solar system objects with the CCB instrument on the GBT. I hope to examine this data and look for similar deviations from a blackbody to those observed for Iapetus. Four of the objects observed are Vesta, Callisto, Ganymede, and Ceres. Vesta (Redman et al. 1992)and Ganymede(Muhleman and Berge 1991) have spectral attributes previously attributed to scattering, whereas Ganymede and Ceres do not. Ganymede and Callisto have icy surfaces, whereas Ceres ${ }^{4}$ and Vesta are not. Thus these four objects provide a perfect combination of icy/not icy and scattering/not scattering objects to search for smaller scale scattering than that observed previously.

For a different project, a substantial amount of RADAR observations of Saturn's icy moons remain un-analyzed. In my postdoctoral work I hope to to examine this data in great detail in order to explore the relationship between brightness temperature and RADAR returns at $2.2 \mathrm{~cm}$, which has already shown some puzzling results

\footnotetext{
${ }^{4}$ Ceres likely contains a substantial amount of water ice, but not on its surface(Zolotov 2009).
} 
in regards to Titan (Janssen et al. 2011), primarily for Iapetus. In addition, I plan to create models simulating the physical surface temperature of the moons of Saturn, so as to gain more insight in the relationship between brightness temperature, physical temperature at depth, and physical surface temperature.

Lastly, with the advent of ALMA and the JVLA, opportunities abound for detailed studies of numerous objects in the solar system. For nearby/large objects (such as icy moons or Pluto), ALMA will actually be capable of resolving their surfaces at wavelengths of $0.6-3 \mathrm{~mm}$, which will allow for the precise mapping of thermal and non-thermal variations across their surfaces and comparison with terrestrial ice emission models. For smaller objects, ALMA will be able to provide measurements of thermal emission, which could be used to determine size, but are much more likely to be dominated by non-thermal effects. The JVLA will allow for $1 \mathrm{~cm}$ measurements which can help provide useful constraints on subsurface structure of icy bodies when combined with ALMA and terrestrial ice models. 


\section{References}

Altenhoff, W. J., Chini, R., Hein, H., Kreysa, E., Mezger, P. G., Salter, C., and Schraml, J. B.: 1988, A\&̈A 190, L15

Aumann, H. H. and Walker, R. G.: 1987, AJ 94, 1088

Bayley, A. M., Davis, R. J., Haggis, J. S., and Karcher, H.: 1994, A\&A 283, 1051

Bell, J. F., Cruikshank, D. P., and Gaffey, M. J.: 1985, Icarus 61, 192

Black, G. J., Campbell, D. B., Carter, L. M., and Ostro, S. J.: 2004, Science 304, 553

Buratti, B. J., Hicks, M. D., Tryka, K. A., Sittig, M. S., and Newburn, R. L.: 2002, Icarus $\mathbf{1 5 5}, 375$

Buratti, B. J. and Mosher, J. A.: 1995, Icarus 115, 219

Burns, J. A., Hamilton, D. P., Mignard, F., and Soter, S.: 1996, in B. A. S. Gustafson and M. S. Hanner (eds.), IAU Colloq. 150: Physics, Chemistry, and Dynamics of Interplanetary Dust, Vol. 104 of Astronomical Society of the Pacific Conference Series, p. 179

Burns, J. A., Lamy, P. L., and Soter, S.: 1979, Icarus 40, 1 
Carvano, J. M., Migliorini, A., Barucci, A., Segura, M., and CIRS Team: 2007, Icarus 187,574

Cassini, G. D.: 1677, Royal Society of London Philosophical Transactions Series I $\mathbf{1 2}, 831$

Chamberlain, M. A., Lovell, A. J., and Sykes, M. V.: 2009, Icarus 202, 487

Chang, A., Gloersen, P., Schmugge, T., Wilheit, T., and Zwally, H.: 1976, J. Glaciology 16, 23

Clark, R. N., Cruikshank, D. P., Jaumann, R., Brown, R. H., Stephan, K., Dalle Ore, C. M., Eric Livo, K., Pearson, N., Curchin, J. M., Hoefen, T. M., Buratti, B. J., Filacchione, G., Baines, K. H., and Nicholson, P. D.: 2012, Icarus 218, 831

Clauser, C. and Huenges, E.: 1995, Thermal Conductivity of Rocks and Minerals, pp 105-126, AGU

Condon, J. J. and Yin, Q. F.: 2001, PASP 113, 362

Conklin, E. K., Ulich, B. L., and Dickel, J. R.: 1977, in Bulletin of the American Astronomical Society, Vol. 9 of Bulletin of the American Astronomical Society, p. 471

Constantikes, K.: 2004, in F. Ochsenbein, M. G. Allen, \& D. Egret (ed.), Astronomical Data Analysis Software and Systems (ADASS) XIII, Vol. 314 of Astronomical Society of the Pacific Conference Series, pp 689-+

Constantikes, K.: 2007, in USNC/CNC/URSI North American Radio Science Meeting in Ottawa, Canada, July 2007

Conway, R. G., Daintree, E. J., and Long, R. J.: 1965, MNRAS 131, 159 
Cook, A. F. and Franklin, F. A.: 1970, Icarus 13, 282

Cotton, W. D., Mason, B. S., Dicker, S. R., Korngut, P. M., Devlin, M. J., Aquirre, J., Benford, D. J., Moseley, S. H., Staguhn, J. G., Irwin, K. D., and Ade, P.: 2009, ApJ 701, 1872

Cotton, W. D., Tody, D., and Pence, W. D.: 1995, A\&AS 113, 159

Coustenis, A., Lellouch, E., Sicardy, B., and Roe, H.: 2010, Earth-Based Perspective and Pre-Cassini-Huygens Knowledge of Titan, p. 9

Cruikshank, D. P., Bell, J. F., Gaffey, M. J., Brown, R. H., Howell, R., Beerman, C., and Rognstad, M.: 1983, Icarus 53, 90

Cruikshank, D. P., Wegryn, E., Dalle Ore, C. M., Brown, R. H., Bibring, J.-P., Buratti, B. J., Clark, R. N., McCord, T. B., Nicholson, P. D., Pendleton, Y. J., Owen, T. C., Filacchione, G., Coradini, A., Cerroni, P., Capaccioni, F., Jaumann, R., Nelson, R. M., Baines, K. H., Sotin, C., Bellucci, G., Combes, M., Langevin, Y., Sicardy, B., Matson, D. L., Formisano, V., Drossart, P., and Mennella, V.: 2008, Icarus 193, 334

Culhane, J. L.: 1991, Nuclear Instruments and Methods in Physics Research A 310, 1

Cuzzi, J. N. and Muhleman, D. O.: 1972, Icarus 17, 548

de Pater, I. and Lissauer, J. J.: 2001, Planetary Sciences

Denk, T., Neukum, G., Roatsch, T., Porco, C. C., Burns, J. A., Galuba, G. G., Schmedemann, N., Helfenstein, P., Thomas, P. C., Wagner, R. J., and West, R. A.: 2010, Science 327, 435 
Dicker, S. R., Korngut, P. M., Mason, B. S., Ade, P. A. R., Aguirre, J., Ames, T. J., Benford, D. J., Chen, T. C., Chervenak, J. A., Cotton, W. D., Devlin, M. J., Figueroa-Feliciano, E., Irwin, K. D., Maher, S., Mello, M., Moseley, S. H., Tally, D. J., Tucker, C., and White, S. D.: 2008, in Society of Photo-Optical Instrumentation Engineers (SPIE) Conference Series, Vol. 7020 of Presented at the Society of Photo-Optical Instrumentation Engineers (SPIE) Conference

Dicker, S. R., Mason, B. S., Korngut, P. M., Cotton, W. D., Compiègne, M., Devlin, M. J., Martin, P. G., Ade, P. A. R., Benford, D. J., Irwin, K. D., Maddalena, R. J., McMullin, J. P., Shepherd, D. S., Sievers, A., Staguhn, J. G., and Tucker, C.: 2009, ApJ 705, 226

Filacchione, G., Capaccioni, F., Ciarniello, M., Clark, R. N., Cuzzi, J. N., Nicholson, P. D., Cruikshank, D. P., Hedman, M. M., Buratti, B. J., Lunine, J. I., Soderblom, L. A., Tosi, F., Cerroni, P., Brown, R. H., McCord, T. B., Jaumann, R., Stephan, K., Baines, K. H., and Flamini, E.: 2012, ArXiv e-prints

Fraser, W. C. and Kavelaars, J. J.: 2009, AJ 137, 72

Hall, R., Goldman, M. A., Parker, D. H., and Payne, J. M.: 1998, in T. G. Phillips (ed.), Society of Photo-Optical Instrumentation Engineers (SPIE) Conference Series, Vol. 3357 of Presented at the Society of Photo-Optical Instrumentation Engineers (SPIE) Conference, pp 265-276

Harlow, R.: 2007, J. of App. Met. and Clim. 46, 23

Heiken, G. H., Vaniman, D. T., and French, B. M.: 1991, Lunar sourcebook - A user's guide to the moon 
Hewison, T., Selbach, N., Heygster, N., Taylor, J., and McGrath, A.: 2002, in Geoscience and Remote Sensing Symposium, pp 2851-2855

Hobbs, M., Jhon, M., and Eyring, H.: 1966, PNAS 56, 31

Howett, C. J. A., Spencer, J. R., Pearl, J., and Segura, M.: 2010, Icarus 206, 573

Hunter, T. R., Mello, M., Nikolic, B., Mason, B. S., Schwab, F. R., Ghigo, F. D., and Dicker, S. R.: 2009, in USNC-URSI National Radio Science Meeting at University of Colorado, January 5-8, 2009

Hunter, T. R., Schwab, F. R., White, S. D., Ford, J. M., Ghigo, F. D., Maddalena, R. J., Mason, B. S., Nelson, J. D., Prestage, R. M., Ray, J., Ries, P., Simon, R., Srikanth, S., and Whiteis, P.: 2011, PASP 123, 1087

Hunter, T. R., Schwab, F. R., White, S. D., Ford, J. M., Ghigo, F. D., Maddalena, R. J., Mason, B. S., Nelson, J. D., Ray, J., and Simon, B.: 2010, in Bulletin of the American Astronomical Society, Vol. 42 of Bulletin of the American Astronomical Society, pp 408-+

Janssen, M. A., Le Gall, A., and Wye, L. C.: 2011, Icarus 212, 321

Janssen, M. A., Lorenz, R. D., West, R., Paganelli, F., Lopes, R. M., Kirk, R. L., Elachi, C., Wall, S. D., Johnson, W. T. K., Anderson, Y., Boehmer, R. A., Callahan, P., Gim, Y., Hamilton, G. A., Kelleher, K. D., Roth, L., Stiles, B., Le Gall, A., and the Cassini Radar Team: 2009, Icarus 200, 222

Jayasuriya, K., Stewart, A., and Campbell, S.: 1985, Journal of Physics and Chemistry of Solids 46(5), 625 
Jewell, P. R. and Prestage, R. M.: 2004, in J. M. Oschmann Jr. (ed.), Society of Photo-Optical Instrumentation Engineers (SPIE) Conference Series, Vol. 5489 of Presented at the Society of Photo-Optical Instrumentation Engineers (SPIE) Conference, pp 312-323

Jewitt, D. C.: 1994, $A J$ 107, 372

Johnson, T. V. and Lunine, J. I.: 2005, Nature 435, 69

Keihm, S. J.: 1984, Icarus 60, 568

Keihm, S. J., Peters, K., Langseth, M. G., and Chute, J. L.: 1973, Earth and Planetary Science Letters 19, 337

Kenyon, S. J., Bromley, B. C., O'Brien, D. P., and Davis, D. R.: 2008, Formation and Collisional Evolution of Kuiper Belt Objects, pp 293-313

Kimura, J., Kawamura, T., Morito, H., Morota, T., Honda, C., Kuramoto, K., and Okada, T.: 2011, Icarus 214, 596

Korngut, P. M., Dicker, S. R., Reese, E. D., Mason, B. S., Devlin, M. J., Mroczkowski, T., Sarazin, C. L., Sun, M., and Sievers, J.: 2010, ArXiv e-prints

Lacasse, R. J.: 1998, in H. Lewis (ed.), Society of Photo-Optical Instrumentation Engineers (SPIE) Conference Series, Vol. 3351 of Presented at the Society of PhotoOptical Instrumentation Engineers (SPIE) Conference, pp 310-319

Linsky, J. L.: 1966, Icarus 5, 606

Marchi, S., Barbieri, C., Dell'Oro, A., and Paolicchi, P.: 2002, A $\& A$ 381, 1059

Marten, A., Hidayat, T., Biraud, Y., and Moreno, R.: 2002, Icarus 158, 532 
Mason, B. S., Dicker, S. R., Korngut, P. M., Devlin, M. J., Cotton, W. D., Koch, P. M., Molnar, S. M., Sievers, J., Aguirre, J. E., Benford, D., Staguhn, J. G., Moseley, H., Irwin, K. D., and Ade, P.: 2010, ApJ 716, 739

Mason, B. S., Weintraub, L., Sievers, J., Bond, J. R., Myers, S. T., Pearson, T. J., Readhead, A. C. S., and Shepherd, M. C.: 2009, ApJ 704, 1433

Matthews, R. A. J.: 1992, QJRAS 33, 253

McCord, T. B., Hansen, G. B., Clark, R. N., Martin, P. D., Hibbitts, C. A., Fanale, F. P., Granahan, J. C., Segura, M., Matson, D. L., Johnson, T. V., Carlson, R. W., Smythe, W. D., and Danielson, G. E.: 1998, J. Geophys. Res. 103, 8603

Miller, C., Verbiscer, A. J., Chanover, N. J., Holtzman, J. A., and Helfenstein, P.: 2011, Icarus 212, 819

Mitchell, D. L. and de Pater, I.: 1994, Icarus 110, 2

Morrison, D., Owen, T., and Soderblom, L. A.: 1986, The satellites of Saturn, pp $764-801$

Moullet, A., Gurwell, M., and Carry, B.: 2010, A\&A 516, L10+

Moullet, A., Lellouch, E., Moreno, R., and Gurwell, M.: 2011, Icarus 213, 382

Mueller, T. G. and Lagerros, J. S. V.: 1998, A\&A 338, 340

Muhleman, D. O.: 1972, Progress in Astronautics and Aeronautics 28, 51

Muhleman, D. O. and Berge, G. L.: 1991, Icarus 92, 263

Muhleman, D. O., Berge, G. L., and Clancy, R. T.: 1984, Science 223, 393 
Müller, T. G. and Barnes, P. J.: 2007, A\&A 467, 737

Müller, T. G., Lellouch, E., Stansberry, J., Kiss, C., Santos-Sanz, P., Vilenius, E., Protopapa, S., Moreno, R., Mueller, M., Delsanti, A., Duffard, R., Fornasier, S., Groussin, O., Harris, A. W., Henry, F., Horner, J., Lacerda, P., Lim, T., Mommert, M., Ortiz, J. L., Rengel, M., Thirouin, A., Trilling, D., Barucci, A., Crovisier, J., Doressoundiram, A., Dotto, E., Gutiérrez, P. J., Hainaut, O. R., Hartogh, P., Hestroffer, D., Kidger, M., Lara, L., Swinyard, B., and Thomas, N.: 2010, A\&\&A 518, L146

Murphy, R. E., Cruikshank, D. P., and Morrison, D.: 1972, ApJ 177, L93

Nikolic, B., Hills, R. E., and Richer, J. S.: 2007a, A\&A 465, 679

Nikolic, B., Prestage, R. M., Balser, D. S., Chandler, C. J., and Hills, R. E.: 2007b, $A \mathscr{S} A 465,685$

O’Neil, K., Balser, D., Bignell, C., Clark, M., Condon, J., McCarty, M., Marganian, P., Shelton, A., Braatz, J., Harnett, J., Maddalena, R., Mello, M., and Sessoms, E.: 2009, in D. A. Bohlender, D. Durand, \& P. Dowler (ed.), Astronomical Society of the Pacific Conference Series, Vol. 411 of Astronomical Society of the Pacific Conference Series, pp 147-+

O’Neil, K., Shelton, A. L., Radziwill, N. M., and Prestage, R. M.: 2006, in C. Gabriel, C. Arviset, D. Ponz, \& S. Enrique (ed.), Astronomical Data Analysis Software and Systems XV, Vol. 351 of Astronomical Society of the Pacific Conference Series, pp $719-+$

Ostro, S. J., West, R. D., Janssen, M. A., Lorenz, R. D., Zebker, H. A., Black, G. J., Lunine, J. I., Wye, L. C., Lopes, R. M., Wall, S. D., Elachi, C., Roth, L., Hensley, 
S., Kelleher, K., Hamilton, G. A., Gim, Y., Anderson, Y. Z., Boehmer, R. A., Johnson, W. T. K., and the Cassini RADAR Team: 2006, Icarus 183, 479

Owen, T. C., Cruikshank, D. P., Dalle Ore, C. M., Geballe, T. R., Roush, T. L., de Bergh, C., Meier, R., Pendleton, Y. J., and Khare, B. N.: 2001, Icarus 149, 160

Parker, D. H. and Payne, J. M.: 1999, in ASPE Proceedings, October 31 - November 5, 1999, Monterey California, 14, p.21-24, pp 21-24

Peterson, C.: 1975, Icarus 24, 499

Porco, C. C., Baker, E., Barbara, J., Beurle, K., Brahic, A., Burns, J. A., Charnoz, S., Cooper, N., Dawson, D. D., Del Genio, A. D., Denk, T., Dones, L., Dyudina, U., Evans, M. W., Giese, B., Grazier, K., Helfenstein, P., Ingersoll, A. P., Jacobson, R. A., Johnson, T. V., McEwen, A., Murray, C. D., Neukum, G., Owen, W. M., Perry, J., Roatsch, T., Spitale, J., Squyres, S., Thomas, P. C., Tiscareno, M., Turtle, E., Vasavada, A. R., Veverka, J., Wagner, R., and West, R.: 2005, Science 307, 1237

Porco, C. C., West, R. A., Squyres, S., McEwen, A., Thomas, P., Murray, C. D., Del Genio, A., Ingersoll, A. P., Johnson, T. V., Neukum, G., Veverka, J., Dones, L., Brahic, A., Burns, J. A., Haemmerle, V., Knowles, B., Dawson, D., Roatsch, T., Beurle, K., and Owen, W.: 2004, Space Sci. Rev. 115, 363

Prestage, R. M., Constantikes, K. T., Balser, D. S., and Condon, J. J.: 2004, in J. M. Oschmann Jr. (ed.), Society of Photo-Optical Instrumentation Engineers (SPIE) Conference Series, Vol. 5489 of Presented at the Society of Photo-Optical Instrumentation Engineers (SPIE) Conference, pp 1029-1040 
Prestage, R. M., Constantikes, K. T., Hunter, T. R., King, L. J., Lacasse, R. J., Lockman, F. J., and Norrod, R. D.: 2009, IEEE Proceedings 97, 1382

Redman, R. O., Feldman, P. A., and Matthews, H. E.: 1998, AJ 116, 1478

Redman, R. O., Feldman, P. A., Matthews, H. E., Halliday, I., and Creutzberg, F.: 1992, AJ 104, 405

Ries, P., Hunter, T. R., Constantikes, K. T., Brandt, J. J., Ghigo, F. D., Mason, B. S., Prestage, R. M., Ray, J., and Schwab, F. R.: 2011, PASP 123, 682

Rivera-Valentin, E. G., Blackburn, D. G., and Ulrich, R.: 2011, Icarus 216, 347

Shirley, Y. L., Mason, B. S., Mangum, J. G., Bolin, D. E., Devlin, M. J., Dicker, S. R., and Korngut, P. M.: 2010, ArXiv e-prints

Showman, A. P. and Malhotra, R.: 1999, Science 296, 77

Simiu, E. and Scanlan, R.: 1996, Wind Effects on Structures, John Wiley and Sons, Inc., New York, NY

Smith, B. A., Soderblom, L., Batson, R. M., Bridges, P. M., Inge, J. L., Masursky, H., Shoemaker, E., Beebe, R. F., Boyce, J., Briggs, G., Bunker, A., Collins, S. A., Hansen, C., Johnson, T. V., Mitchell, J. L., Terrile, R. J., Cook, A. F., Cuzzi, J. N., Pollack, J. B., Danielson, G. E., Ingersoll, A. P., Davies, M. E., Hunt, G. E., Morrison, D., Owen, T., Sagan, C., Veverka, J., Strom, R., and Suomi, V. E.: 1982, Science 215, 504

Smith, D. R., Paglione, T. A., Lovell, A. J., Ukita, N., and Matsuo, H.: 2000, in H. R. Butcher (ed.), Society of Photo-Optical Instrumentation Engineers (SPIE) 
Conference Series, Vol. 4015 of Presented at the Society of Photo-Optical Instrumentation Engineers (SPIE) Conference, pp 467-475

Spencer, J. R. and Denk, T.: 2010, Science 327, 432

Spencer, J. R., Lebofsky, L. A., and Sykes, M. V.: 1989, Icarus 78, 337

Stansberry, J., Grundy, W., Brown, M., Cruikshank, D., Spencer, J., Trilling, D., and Margot, J.-L.: 2008, Physical Properties of Kuiper Belt and Centaur Objects: Constraints from the Spitzer Space Telescope, pp 161-179

Stansberry, J. A., Pisano, D. J., and Yelle, R. V.: 1996, Planet. Space Sci. 44, 945

Steppe, H., Jeyakumar, S., Saikia, D. J., and Salter, C. J.: 1995, A $6 A S$ 113, 409

Stilburn, J., Fletcher, M., Leckie, B., Barrick, G., and Monnet, G.: 1997, PASP 109, 1165

Stilburn, J. R.: 1992, PASP 104, 955

Symmes, A., Anderson, R., and Egan, D.: 2008, in Society of Photo-Optical Instrumentation Engineers (SPIE) Conference Series, Vol. 7012 of Presented at the Society of Photo-Optical Instrumentation Engineers (SPIE) Conference

Tabak, R. G. and Young, W. M.: 1989, Earth Moon and Planets 44, 251

Tamayo, D., Burns, J. A., Hamilton, D. P., and Hedman, M. M.: 2011, Icarus 215, 260

Thomas, P. C.: 2010, Icarus 208, 395 
Thomas, P. C., Burns, J. A., Helfenstein, P., Squyres, S., Veverka, J., Porco, C., Turtle, E. P., McEwen, A., Denk, T., Giese, B., Roatsch, T., Johnson, T. V., and Jacobson, R. A.: 2007, Icarus 190, 573

Tikhonov, V., Boyarskii, D. A., Polyakova, O. N., Dzardanov, A. L., and Gol'tsman, G.: 2010, Progress In Electromagnetics Research B 25, 349

Tikhonova, T. V. and Troitski, V. S.: 1969, Soviet Ast. 13, 120

Tryka, K. A., Brown, R. H., Chruikshank, D. P., Owen, T. C., Geballe, T. R., and Debergh, C.: 1994, Icarus 112, 513

van Helden, A.: 1984, in T. Gehrels and M. S. Matthews (eds.), Saturn, pp 23-43

Vangasse, P., Charlton, J., and Jarrett, M.: 1996, Advances in Space Research 17, 75

Verbiscer, A., French, R., Showalter, M., and Helfenstein, P.: 2007, Science 315, 815

Verbiscer, A. J., Skrutskie, M. F., and Hamilton, D. P.: 2009, Nature 461, 1098

Weiland, J. L., Odegard, N., Hill, R. S., Wollack, E., Hinshaw, G., Greason, M. R., Jarosik, N., Page, L., Bennett, C. L., Dunkley, J., Gold, B., Halpern, M., Kogut, A., Komatsu, E., Larson, D., Limon, M., Meyer, S. S., Nolta, M. R., Smith, K. M., Spergel, D. N., Tucker, G. S., and Wright, E. L.: 2011, ApJS 192, 19

Wells, D. C.: 2000, in N. Manset, C. Veillet, \& D. Crabtree (ed.), Astronomical Data Analysis Software and Systems IX, Vol. 216 of Astronomical Society of the Pacific Conference Series, pp 355-+

Weng, F., Fang, Y., and Grody, N.: 2001, J. Geophys. Res. 106, 20,115

Wiesmann, A. and Mätzler, C.: 1999, Remote. Sens. Environ. 70, 307 
Wiesmann, A., Mätzler, C., and T., W.: 1998, Radio Sci. 33, 273

Wilson, P. D. and Sagan, C.: 1996, Icarus 122, 92

Wright, E. L., Chen, X., Odegard, N., Bennett, C. L., Hill, R. S., Hinshaw, G., Jarosik, N., Komatsu, E., Nolta, M. R., Page, L., Spergel, D. N., Weiland, J. L., Wollack, E., Dunkley, J., Gold, B., Halpern, M., Kogut, A., Larson, D., Limon, M., Meyer, S. S., and Tucker, G. S.: 2009, ApJS 180, 283

Yoder, C. F.: 1995, in T. J. Ahrens (ed.), Global Earth Physics: A Handbook of Physical Constants, p. 1

Zolotov, M. Y.: 2009, Icarus 204, 183 